University of Louisville

ThinkIR: The University of Louisville's Institutional Repository

Electronic Theses and Dissertations

8-2017

\title{
A smart highway work zone merge system enabled by connected vehicles.
}

Abdulmaged Algomaiah

University of Louisville

Follow this and additional works at: https://ir.library.louisville.edu/etd

Part of the Transportation Engineering Commons

\section{Recommended Citation}

Algomaiah, Abdulmaged, "A smart highway work zone merge system enabled by connected vehicles." (2017). Electronic Theses and Dissertations. Paper 2783.

https://doi.org/10.18297/etd/2783

This Master's Thesis is brought to you for free and open access by ThinkIR: The University of Louisville's Institutional Repository. It has been accepted for inclusion in Electronic Theses and Dissertations by an authorized administrator of ThinkIR: The University of Louisville's Institutional Repository. This title appears here courtesy of the author, who has retained all other copyrights. For more information, please contact thinkir@louisville.edu. 


\title{
A SMART HIGHWAY WORK ZONE MERGE SYSTEM ENABLED BY CONNECTED VEHICLES
}

By

Abdulmaged Algomaiah

B.S., Queensland University of Technology, 2014

\author{
A Thesis \\ Submitted to the Faculty of \\ J.B. Speed School of Engineering at the University of Louisville \\ in Partial Fulfillment of the Requirements \\ for the Degree of \\ Master of Science \\ in Civil Engineering \\ Department of Civil \& Environmental Engineering \\ University of Louisville \\ Louisville, Kentucky
}

August 2017 


\section{Copyright 2017 by Abdulmaged Algomaiah}

All rights reserved 

A SMART HIGHWAY WORK ZONE MERGE SYSTEM ENABLED BY CONNECTED VEHICLES

By

Abdulmaged Algomaiah

B.S., Queensland University of Technology, 2014

A Thesis Approved on

July 20,2017

by the following Thesis Committee:

Dr. Zhixia (Richard) Li

Dr. Zhihui Sun

Dr. Jacqueline McNeil 


\section{DEDICATION}

To all those who

appreciate, support and inspire Majeed and they know who they are to me. 


\section{ACKNOWLEDGMENTS}

I would like to thank my supervisor Dr. Richard (Zhixia) Li for his constant support and motivation from early stage of this thesis till the last moment. I am deeply thankful for

the time he spent on sharing his knowledge and experience. He has always been a source of encouragement and profound guidance.

I am sincerely grateful to Dr. Zhihui Sun and Dr. Jacqueline McNeil for their participation in the committee and their valuable feedback. I also would like to deeply thank Dr. J.P Mohsen for his advice and ultimate support during last two years. 


\title{
ABSTRACT \\ A SMART HIGHWAY WORK ZONE MERGE SYSTEM ENABLED BY CONNECTED VEHICLES
}

\author{
Abdulmaged Algomaiah
}

July 20, 2017

Typical traffic control and operations at work zones usually are not very effective in mitigating the work zone bottleneck. A major reason is that the common early merge behaviors limits utilizing the available capacity in the closed lane, especially under moderate to high traffic demands. Recently, using late merge strategy is encouraged to prevent early merge behavior and fully utilize the closed lane and therefore increase capacity of work zone. The development of late merge strategy was not enough to substantially improve the situation due to the lack of compliance and communication among drivers. This thesis suggests and investigates a smart work zone merge system enabled by connected vehicles. The main principle is to enhance the opportunity of cooperative late merge via the new technology, connected vehicles $(\mathrm{CV})$. When connected vehicles share information and negotiate the best movements for the benefit of traffic flow, drivers will be able to receive merging suggestions. The system is called Cooperative Late Merge System (CLMS) where the layout is quiet similar to late merge system with additional advanced elements. The CLMS has been introduced and investigated by using simulation software package (VISSIM). Microscopic car-following 
and lane-changing models were selected and updated to fit with the main goal of the system and the purpose of this study. This paper uses Hidas (2005) lane-changing model and car-following model (Wiedemann 99) provided by VISSIM. Simulation experiment was designed to include a traditional work zone system and a late merge system in order to compare them with the performance of the CLMS. The experiment design considered eight different variables with multiple levels. Simulation output included major measures of effectiveness (MOEs) including throughput, capacity, V/C ratio, delay and queue length. The CLMS has demonstrated an improved traffic operation performance at all considered MOEs, especially, in the case of moderate traffic demand. The system is capable of increasing throughput and capacity of work zone as well as decreasing the delay and queue length at most of traffic conditions. 


\section{TABLE OF CONTENTS}

ACKNOWLEDGMENTS ........................................................................... iv

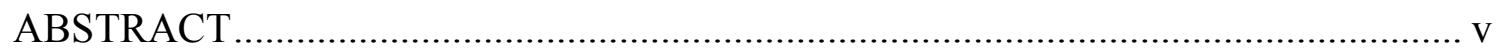

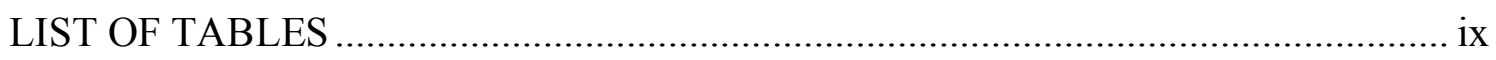

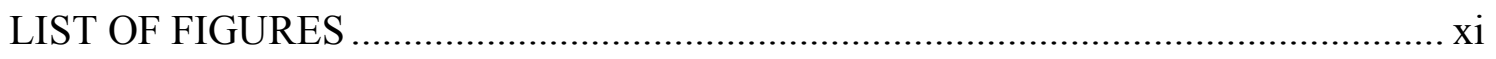

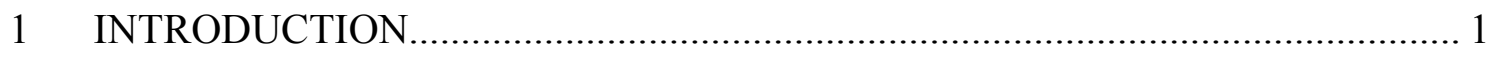

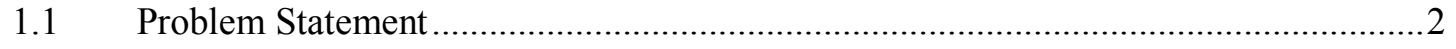

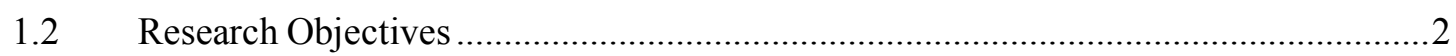

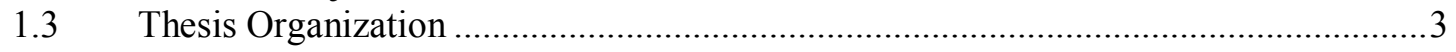

2 LITERATURE REVIEW ............................................................................ 4

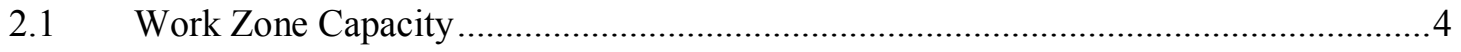

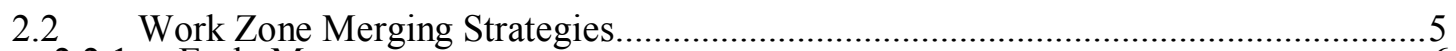

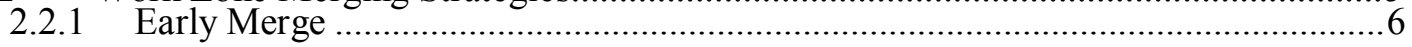

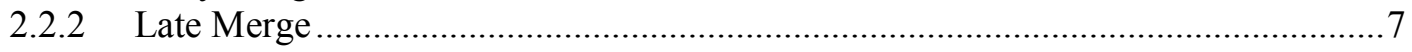

2.2.3 Dynamic Merge Systems in Practice .................................................................. 9

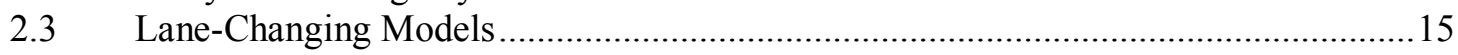

2.4 Connected Vehicles Applications in Work Zone ................................................... 18

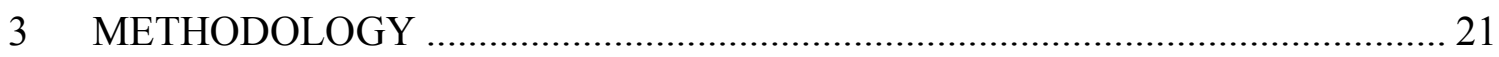

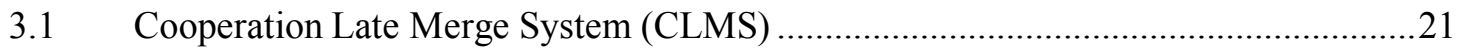

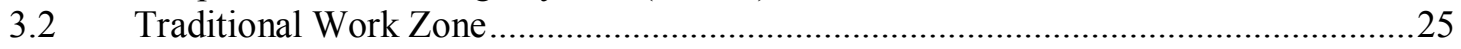

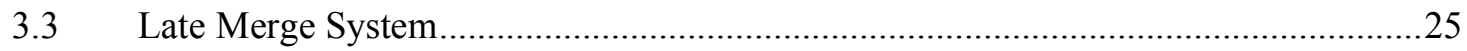

3.4 CLMS Level 1: Decentralized Cooperative Merge .....................................................26

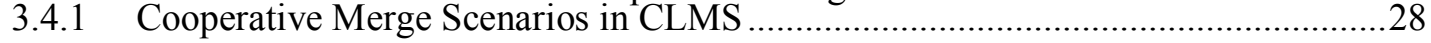

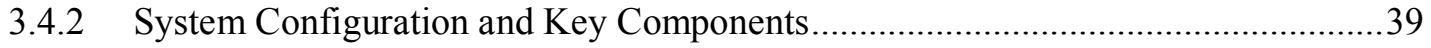

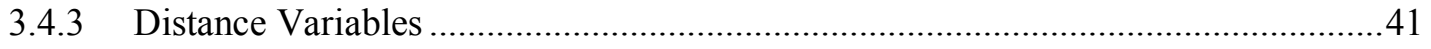

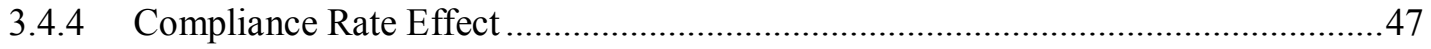

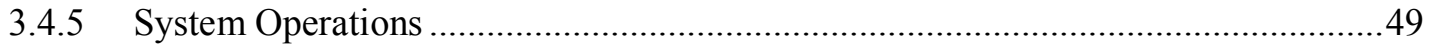

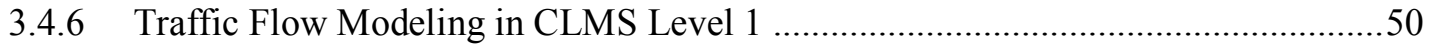

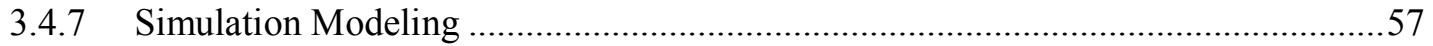

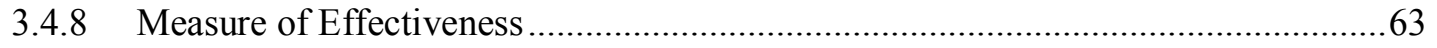

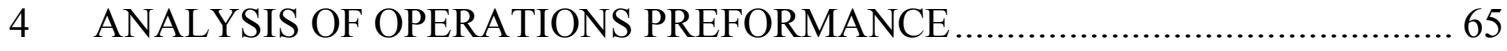

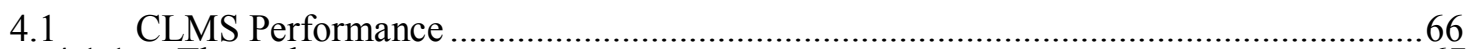

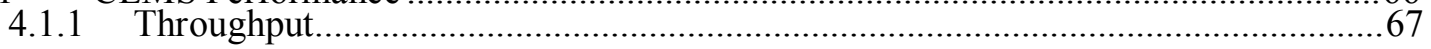

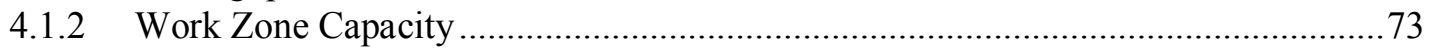

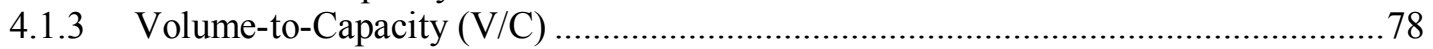

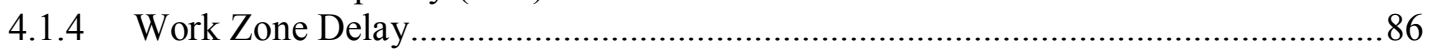

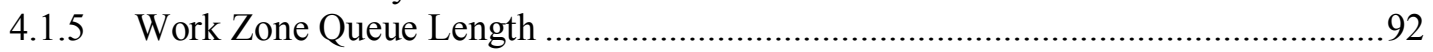


4.2 Effect of Compliance Rate

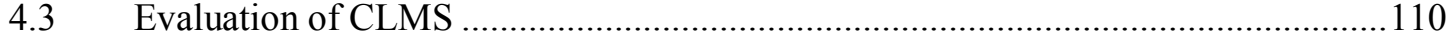

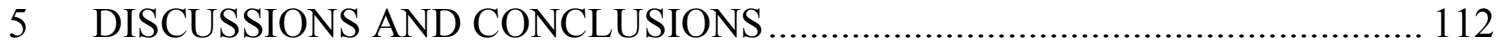

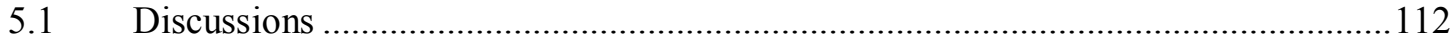

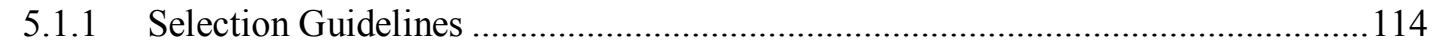

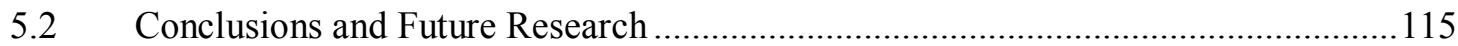

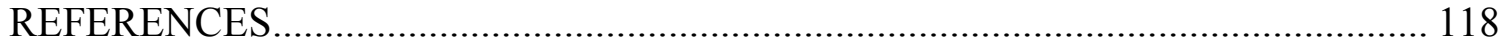

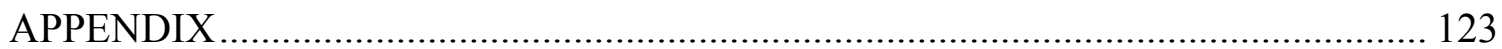

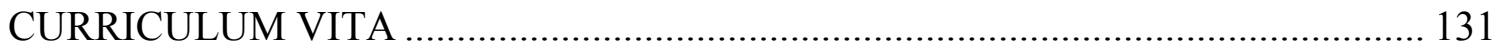




\section{LIST OF TABLES}

Table 1 Summary of the Distances for Late Merge Systems ........................................ 14

Table 2 Summary of Scenarios Considered in CLMS .................................................... 29

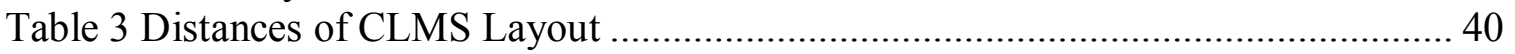

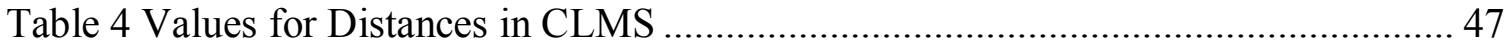

Table 5 Compliance Rates Considered in CLMS ........................................................ 49

Table 6 Parameters of VISSIM Car-following Model (PTV, 2016) ............................... 52

Table 7 Parameters in Hidas Lane_Changing Model .................................................... 54

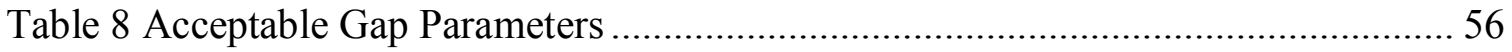

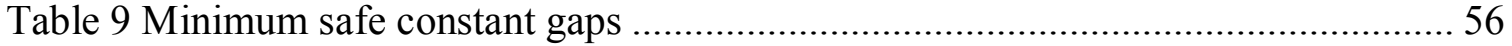

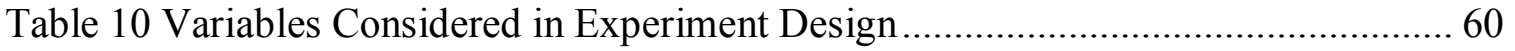

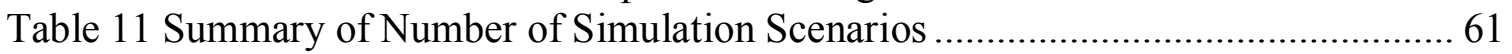

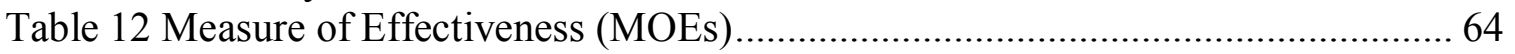

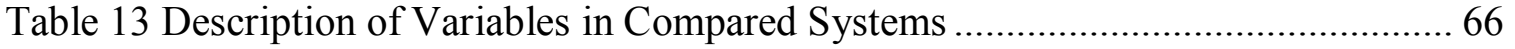

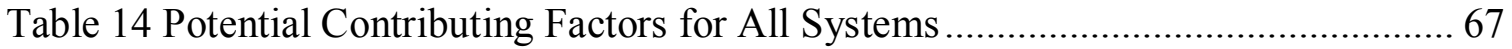

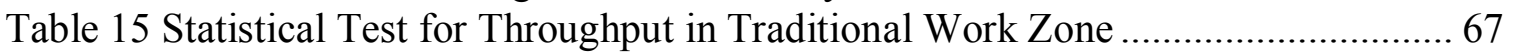

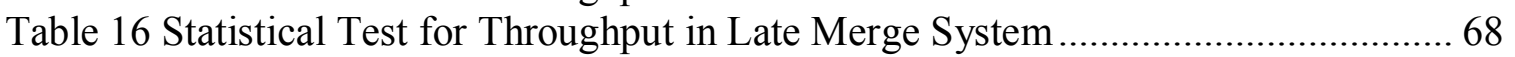

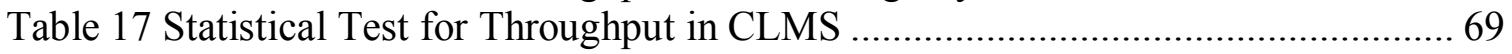

Table 18 Summary of Significant Variables Impacting Throughput.............................. 70

Table 19 T-Test Results for the Difference in Throughput for All Systems ................... 72

Table 20 Statistical Test for Capacity in Traditional Work Zone ................................... 73

Table 21 Statistical Test for Capacity in Late Merge System ...................................... 74

Table 22 Statistical Test for Capacity in CLMS ....................................................... 75

Table 23 Summary of Significant Variables Impacting Capacity ................................. 76

Table 24 T-Test Results for the Difference in Capacity ................................................ 77

Table 25 Statistical Test for $0.9 \mathrm{~V} / \mathrm{C}$ in Traditional Work Zone .................................... 78

Table 26 Statistical Test for $0.9 \mathrm{~V} / \mathrm{C}$ in Late Merge System ......................................... 79

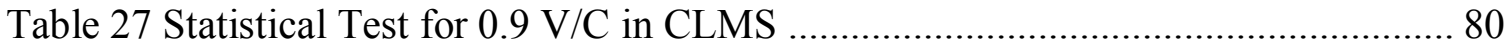

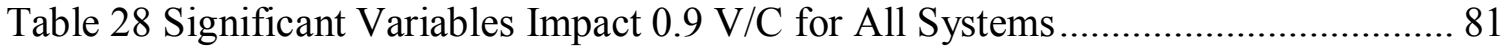

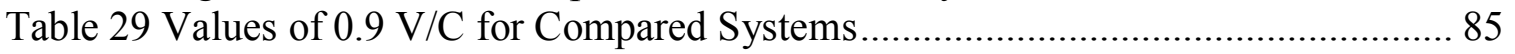

Table 30 Statistical Test for Delay in Traditional Work Zone ....................................... 86

Table 31 Statistical Test for Delay in Late Merge System ........................................... 87

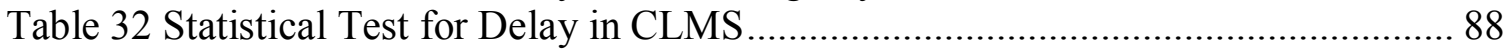

Table 33 Significant Variables Impact Delay for All Systems ...................................... 89

Table 34 T-Test Results for the Difference of Delay .................................................. 91

Table 35 Statistical Test for Queue Length in Traditional Work Zone ............................ 92

Table 36 Statistical Test for Queue Length in Late Merge System ............................... 93

Table 37 Statistical Test for Queue Length in CLMS ................................................ 94 
Table 38 Significant Variables Impact Queue Length for All Systems..... 95

Table 39 T-Test Results for the Difference of Queue Length ....................................... 97

Table 40 Variables Considered in Compliance Rate Effect .......................................... 98

Table 41 Significant Variables Impact Compliance Rate on CLMS .............................. 99

Table 42 T-Test of Compliance Rate Effect on Throughput When $\sigma_{\text {Non-CV }}=0.3 \ldots \ldots \ldots . .102$

Table 43 T-Test of Compliance Rate Effect on Throughput When $\sigma$ Non-CV $=0.6 \ldots \ldots \ldots . . .103$

Table 44 T-Test of Compliance Rate Effect on Delay When $\sigma_{\text {Non-CV }}=0.3 \ldots \ldots \ldots \ldots \ldots \ldots . . . . .106$

Table 45 T-Test of Compliance Rate Effect on Delay When $\sigma_{\text {Non-CV }}=0.6 \ldots \ldots \ldots \ldots \ldots \ldots . . . . . .107$

Table 46 T-Test of Compliance Rate Effect on Queue Length When $\sigma_{\text {Non-CV }}=0.3 \ldots . . .109$

Table 47 T-Test of Compliance Rate Effect on Queue Length When $\sigma$ Non-CV = 0.6.. 110

Table 48 Selection Guide for Work Zone Control Strategy ........................................ 114 


\section{LIST OF FIGURES}

Figure 1 Early Merge System by INDOT (Tarko et al., 2001) ....................................... 7 Figure 2 Late Merge System by Pennsylvania DOT and Minnesota DOT (McCoy et al., 2001)

Figure 3 Late Merge System by Maryland SHA and Michigan DOT (Kang et al., 2006)11

Figure 4 Modified Late Merge Design Suggested by Grillo (2008) ............................. 12

Figure 5 Modified Late Merge Design Suggested by (Redwan et al., 2009) ................... 13

Figure 6 Description of Three Stages of CLMS ............................................................ 23

Figure 7 Major components of Level 1 CLMS ........................................................... 24

Figure 8 Flowchart of the Logic of CLMS in Work Zone Area ................................... 27

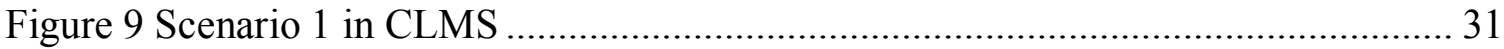

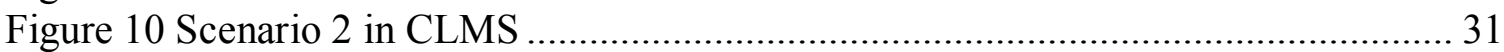

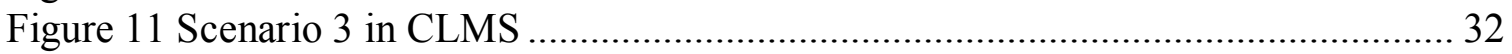

Figure 12 Flowchart of the CLMS Logic in Downstream Scenarios ............................. 33

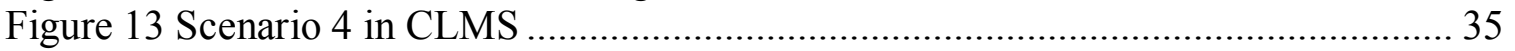

Figure 14 Flowchart of the CLMS Logic in Upstream Scenarios ................................. 35

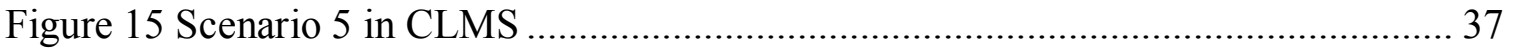

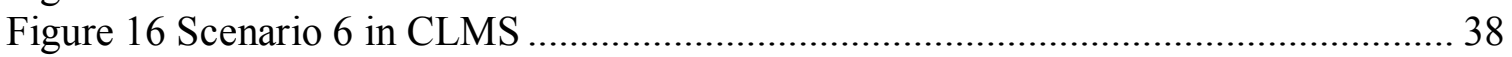

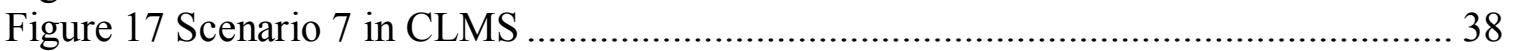

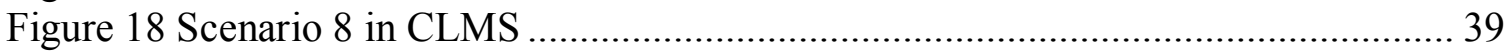

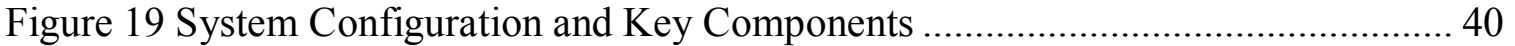

Figure 20 Summary of Lane-changing Models in CLMS .......................................... 57

Figure 21 The Actual Site of Simulated Experiment (I-64 East) ................................. 58

Figure 22 Comparison of Throughput in All Systems ............................................... 71

Figure 23 Comparison of Capacity for All Systems ..................................................... 76

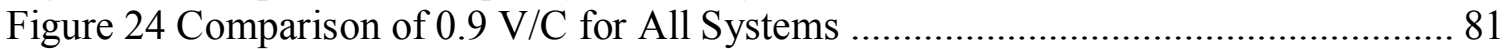

Figure 25 Comparison of $\mathrm{V} / \mathrm{C}$ for All Systems When Lane Distribution is $30 \% \ldots \ldots \ldots \ldots . . .82$

Figure 26 Comparison of V/C for All Systems When Lane Distribution is 50\%........... 83

Figure 27 Comparison of V/C for All Systems When Lane Distribution is $70 \% \ldots \ldots \ldots \ldots . .84$

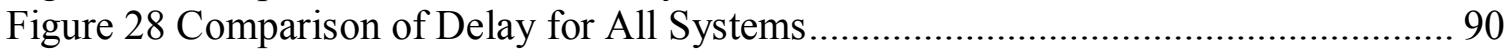

Figure 29 Comparison of Queue Length for All Systems ........................................... 96

Figure 30 CV Compliance Rate Effect on Throughput in CLMS ................................ 101

Figure 31 Compliance Rates Effect on Capacity in CLMS ......................................... 104

Figure 32 CV Compliance Rate Effect on Delay in CLMS....................................... 105

Figure 33 Non-CV Compliance Rate Effect on Queue Length in Late Merge System.. 108 


\section{CHAPTER I}

\section{INTRODUCTION}

Highway work zones are a major bottleneck in highway systems because of the sudden reduced capacity due to dropped lanes. Therefore, work zones can cause serious congestion and safety issues. In practice, the typical traffic control and operations at work zones usually are not very effective in mitigating the work zone bottleneck. A major reason is that the common early merge behaviors limit utilizing the available capacity in the closed lane especially under moderate to high traffic demands. Researchers encouraged using late merge strategy in congested conditions by fully utilizing the closed lane till the work zone tapper in order to increase the throughput at the work zone (Pesti et al., 1999; McCoy, et al., 2001; Beacher et al., 2005; Redwan et al., 2009). However, in reality, effective implementation of late merge is usually restricted by the low driver compliance rate, which is attributed to the dynamic driver's behaviors. Without communication between vehicles, drivers' maneuvers in the merge area are based on individual behaviors and aggressiveness. Although late merge theoretically would improve the merge efficiency, the lack of information exchange and coordination restrains this strategy from being effective when implemented in the field. This thesis will introduce a study that is dedicated to improving the aforementioned work zone capacity issue by implementing the Connected Vehicles (CV) technology. It proposes 
Cooperative Late Merge System (CLMS) and evaluate the new system using microscopic traffic simulation software (VISSIM). The performance of the proposed system that utilizes CV technology is compared with ordinary late merge work zone and traditional work zone.

\subsection{Problem Statement}

There is a lack of information exchange and coordination between drivers in the merging process at work zones, which restricts the benefit of late merge system and thus reduces the capacity of the work zone.

\subsection{Research Objectives}

The objectives of the study are the following:

- Develop late merge system (CLMS) and update the lane-changing microscopic models to reflect the $\mathrm{CV}$ technology;

- Evaluate the performance of the CLMS compared to ordinary late merge system and traditional work zone;

- Test different compliance rate and market penetration rate;

- Develop guidelines about selection of appropriate work zone traffic control strategy that can be considered by transportation agencies. 


\subsection{Thesis Organization}

The thesis starts with a literature review that covers work zone capacity, merging strategies and CV technology in work zone. The chapter investigates the conducted studies in improving the capacity of work zone, in developing merge strategies and in implementing $\mathrm{CV}$ in work zone. Then, it is followed by a methodology chapter that describes the layout of the CLMS, traffic modeling, simulation modeling and measure of effectiveness. The results are then analyzed in the operations performance chapter in terms of capacity, overall delay and queue length. The last chapter is conclusions and recommendations, which draws the thesis to a close. 


\section{CHAPTER II}

\section{LITERATURE REVIEW}

The major goal of freeways is to facilitate the flow of traffic by providing uninterrupted movement along the road. Work zones prevent the benefits by reducing the capacity of the freeway and slowing the traffic. However, eliminating work zones is not an option due to the demand of frequent maintenance and rehabilitation of freeways. Therefore, there have been early attempts to mitigate the effect of work zone and increase its capacity.

\subsection{Work Zone Capacity}

During late 1970s and early 1980s, several studies examined the capacity of work zones with minimal traffic control devices (Al-Kaisy, Zhou and Hall 2000). Dudek and Richards (1982) conducted studies in Houston and Dallas, and found that the average capacity of work zones varies with the freeway number of lanes and number of closed lanes. A three-lane section with one closed lane resulted an average of $1500 \mathrm{veh} / \mathrm{hr} / \mathrm{ln}$, while a two-lane freeway section with one closed lane generated $1130 \mathrm{veh} / \mathrm{hr} / \mathrm{ln}$. The study also observed that the layout of work zones and type of construction influence work zone capacity. This is supported by the Highway Capacity Manual (HCM 2000) as it found different capacity results by different studies. 
Krammes and Lopez (1992) found a model of work zone capacity that has several adjustment factors. Work zone capacity in their model is given by:

$$
C=1600+(I-R) \times H \times N
$$

Where:

$I$ is the adjustment factor related to work zone activity;

$R$ is the adjustment factor related to ramps;

$H$ is the adjustment factor for heavy vehicles, and

$N$ is the number of open lanes in the work zone.

Dixon et al. (1996) claimed that the capacity of work zones is related to the merging behavior of upstream and downstream (at the taper). The difference between the two types of merging and its influence on capacity encouraged researchers to explore merging strategies, particularly, early merge and late merge concepts.

\subsection{Work Zone Merging Strategies}

In conventional work zones, the advanced warning sign of the closed lane is far from the taper. Drivers tend to merge to the open lane from the moment they realize which lane is closed. Such behavior significantly increases the traffic in the open lane, while the closed 
lane remains unutilized. With the high traffic volume, the immediate result is traffic back up in the open lane. This problem encouraged researchers to consider employing different strategies to improve work zone mobility. The main two strategies are Early Merge (EM) and Late Merge (LM).

\subsubsection{Early Merge}

Nemeth and Rouphail (1982) tested a new simulation modeling and concluded that early merge can significantly prevent the number of forced merges in the case of high traffic volume. Mousa et al. (1990) also tested another simulation modeling and found that travel times were escalated due to the existence of slower vehicles that cause higher delay for a longer distance than if vehicles were divided between the two lanes till the taper. The authors claimed that this may enhance the probability of queue jumping. A practical application of early merge was investigated by Tarko et al (2001) and implemented by Indiana Department of Transportation (INDOT). The system considered using variable messaging signs to instruct drivers to merge early. These signs were activated to flash the message "No Passing When Flashing," by using sensors. Each device was responsible for activating the upcoming sign upstream of the established queue. By merging early, the results revealed a lower number of aggressive maneuvers during the merge process. The researchers emphasized that this strategy was advantageous with low to moderate traffic volume. 


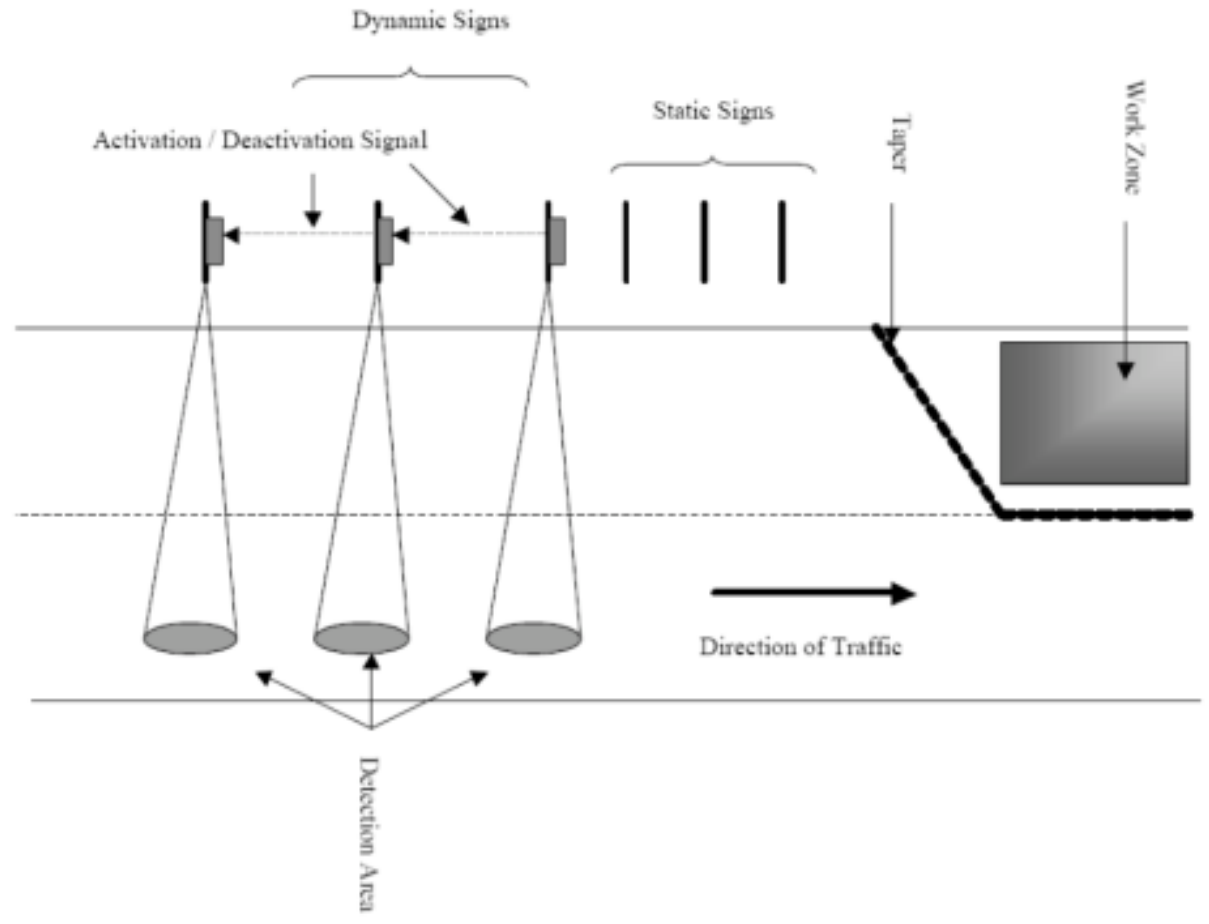

Figure 1 Early Merge System by INDOT (Tarko et al., 2001)

\subsubsection{Late Merge}

Pesti et al. (1999) investigated the traffic flow characteristics of late merge work zone control strategy. A field study took a place in a late merge work zone on northbound I-79 north of Canonsburg, Pennsylvania. The two-lane corridor dropped to one-lane and the data collected using videotape recording for 4 days. The evaluation results from the field data indicated that LM is more effective than the conventional merge system. Work zone capacity using LM was increased by $18 \%$ comparing to the conventional merge. 
However, the study indicated that to get the most benefit out of LM, drivers have to fully understand the system and comply with it.

McCoy, et al. (2001) proposed a hybrid design of conventional merge and dynamic late merge that changes depending on the traffic conditions. When traffic volume is low or moderate, the speed is relatively high and late merge may cause confusion to the drivers, which increase the chances of crashes. Therefore, the researchers suggested using conventional merge during off-peak and switching to late merge during on-peak. In their system, the dynamic late merge consisted of a series of advance signs that are activated when congested is detected by the sensors and the signs would then instruct the drivers to use both lanes till the merge point. The signs would be deactivated and merge would return to the conventional method when the sensors detected the decongestion. Beacher et al. (2005) investigated the performance of late merge comparing to conventional merge using simulation models developed by VISSIM for both type of traffic controls. Conventional and late merge systems were tested under different traffic volumes, heavy vehicle percentage, lane closure configurations and desired speed. The researchers found that the use of late merge is recommended in the case of two-lane freeway with one closed lane and three-lane freeway with two closed lanes closure configuration. 


\subsubsection{Dynamic Merge Systems in Practice}

In practice, there are several late merge systems applied with different elements and different configurations. Since early 2000s, Department of Transportation for several states tested late merge system including, but not limited to Pennsylvania, Minnesota, Maryland, Michigan and Florida.

\subsubsection{Pennsylvania DOT and Minnesota DOT Practices}

The Dynamic Late Merge (DLM) was purposed by (McCoy et al., 2001), which aimed to reduce congestion and delay. The system warns drivers about work zone 2 miles before the taper. The first "USE BOTH LANES TO MERGE POINT SIGN" is placed 1.5 miles from the taper. 350 feet from the taper, the sign "MERGE HERE - TAKE YOUR TURN" is located. Other static signs suggested by MUTCD such as "ROAD WORK 1 MILE" and "LEFT LANE CLOSED 1500 FT", are distributed along the road. All signs in this system are places in both sides of the freeway. This configuration is applied by Pennsylvania DOT and Minnesota DOT (see Figure 1). 


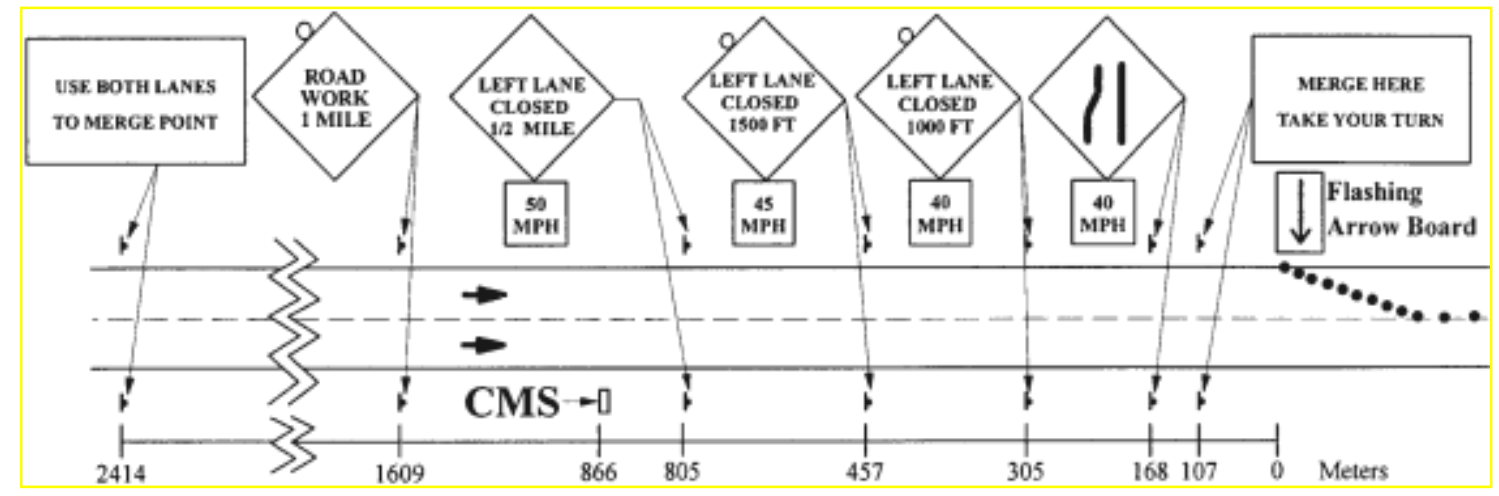

Figure 2 Late Merge System by Pennsylvania DOT and Minnesota DOT (McCoy et al., 2001)

Pennsylvania DOT field study reported incompliance cases from both passenger cars and trucks. However, higher percentages remained in the closed lane, which indicates that most of drivers comply with late merge system. Minnesota DOT report found that the use of the closed lane increased by $30-40 \%$ during on-peak, while increased by $60 \%$ during off-peak. The system tested by Pennsylvania DOT resulted an increase in throughput from 1460 to 1540 pcph (Pesti et al., 1999). Minnesota DOT observed an increase in throughput from 1500 to $1560 \mathrm{vph}$ (URS, 2003).

\subsubsection{Maryland DOT Practice}

The static late merge designed by Maryland State Highway Administration warns drivers about work zone about 3 miles before the taper and advise them to "USE BOTH LANES TO MERGE POINT" 2 miles before the taper on both sides of the freeway. Drivers are asked to merge 500 feet before the taper by using a sign on both sides of the 
road stating "MERGE HERE -TAKE YOUR TURN". Other static signs suggested by

MUTCD such as "ROAD WORK 1 MILE” and "RIGHT LANE CLOSED 1/2 MILE”, are

distributed along the road. A late merge design with the same spacing was also applied by Michigan DOT (see Figure 3).

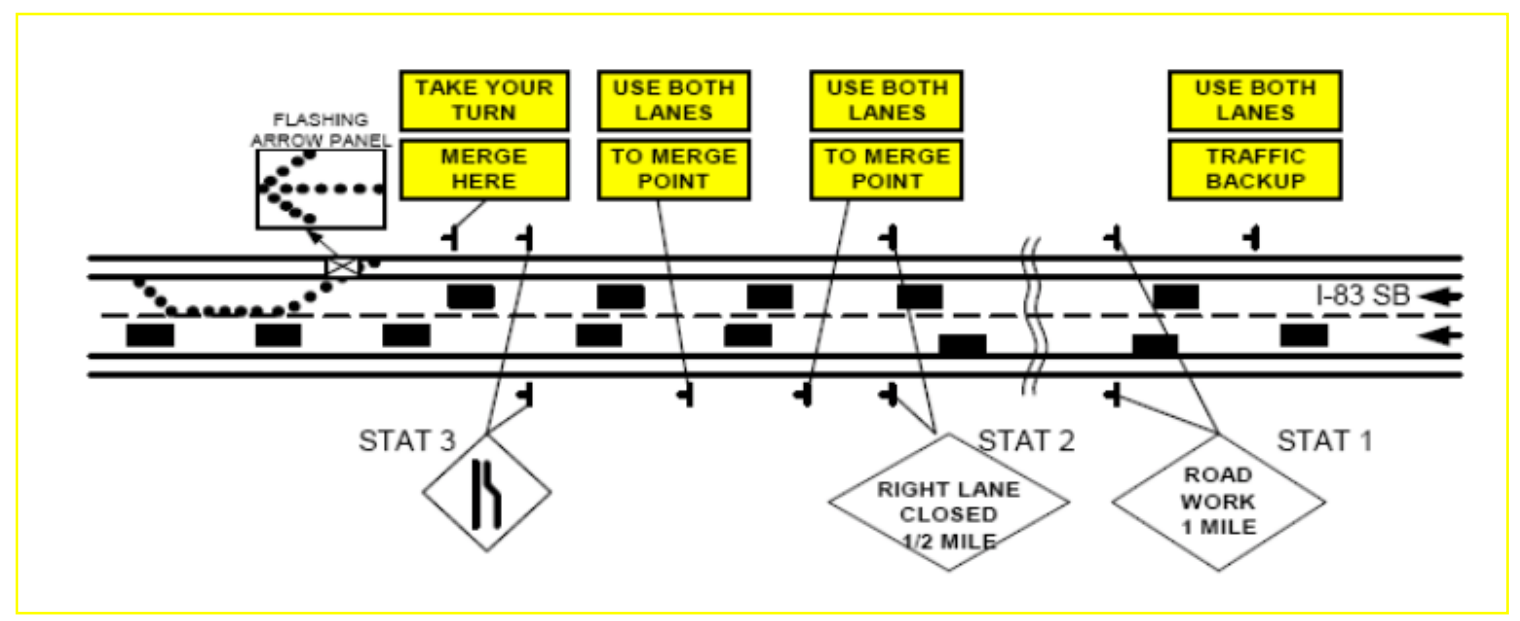

Figure 3 Late Merge System by Maryland SHA and Michigan DOT (Kang et al., 2006)

Maryland SHA applied the system in two different sites and reported an average increase in throughput from 1505 to $1718 \mathrm{vph}$ (Kang et al., 2006).

\subsubsection{Michigan DOT Practice}

Grillo et al. (2008) purposed a system that advices drivers to about the work zone about 3 miles before the taper. Late merge is advices about 2 miles before the taper with one sign on the closed lane side stating "USE BOTH LANES" and another one 1.5 miles before 
the taper stating "STAY IN YOUR LANE" on the closed side as well. The s ign "MERGE HERE - TAKE YOUR TURN" is located 1500 feet from the taper on the closed lane. Other static signs suggested by MUTCD such as "ROAD WORK AHEAD" and "LEFT LANE CLOSED AHEAD", are distributed along the road. The system is applied by Michigan DOT in several sites including Interstate 94 .

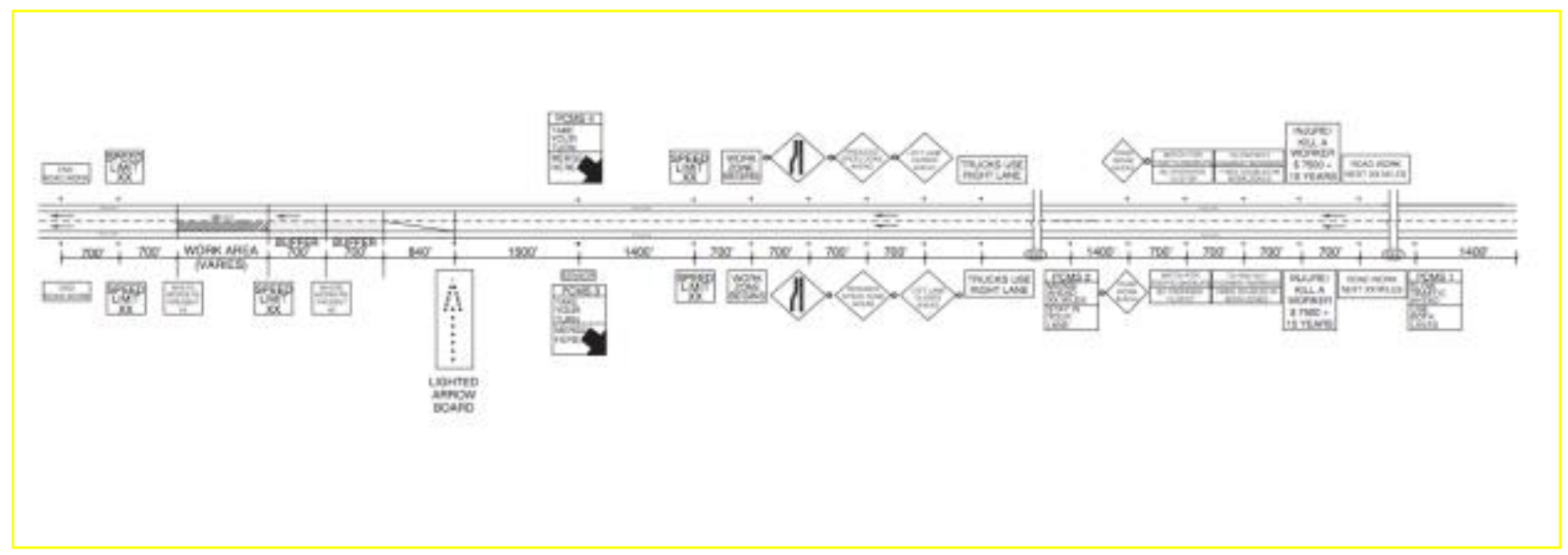

Figure 4 Modified Late Merge Design Suggested by Grillo (2008)

Michigan DOT applied a field study in different sites and found that the compliance rate is approximately between $55-65 \%$. An increase in throughput from 990 to $1207 \mathrm{vph}$ was reported in average (Grillo et al, 2008). 


\subsubsection{Florida DOT Practice}

Redwan et al. (2009) modified the dynamic late merge to warn drivers 3 miles before the taper and advise them to "MERGE AHEAD" 1.5 miles. Both signs are placed on the closed lane side of the freeway. The merge advising sign is located 1000 feet from the taper. Other static signs suggested by MUTCD such as "ROAD WORK 1 MILE" and "RIGHT LANE CLOSED 1/2 MILE", are distributed along the road. This modifies late merge system is applied by Florida DOT.

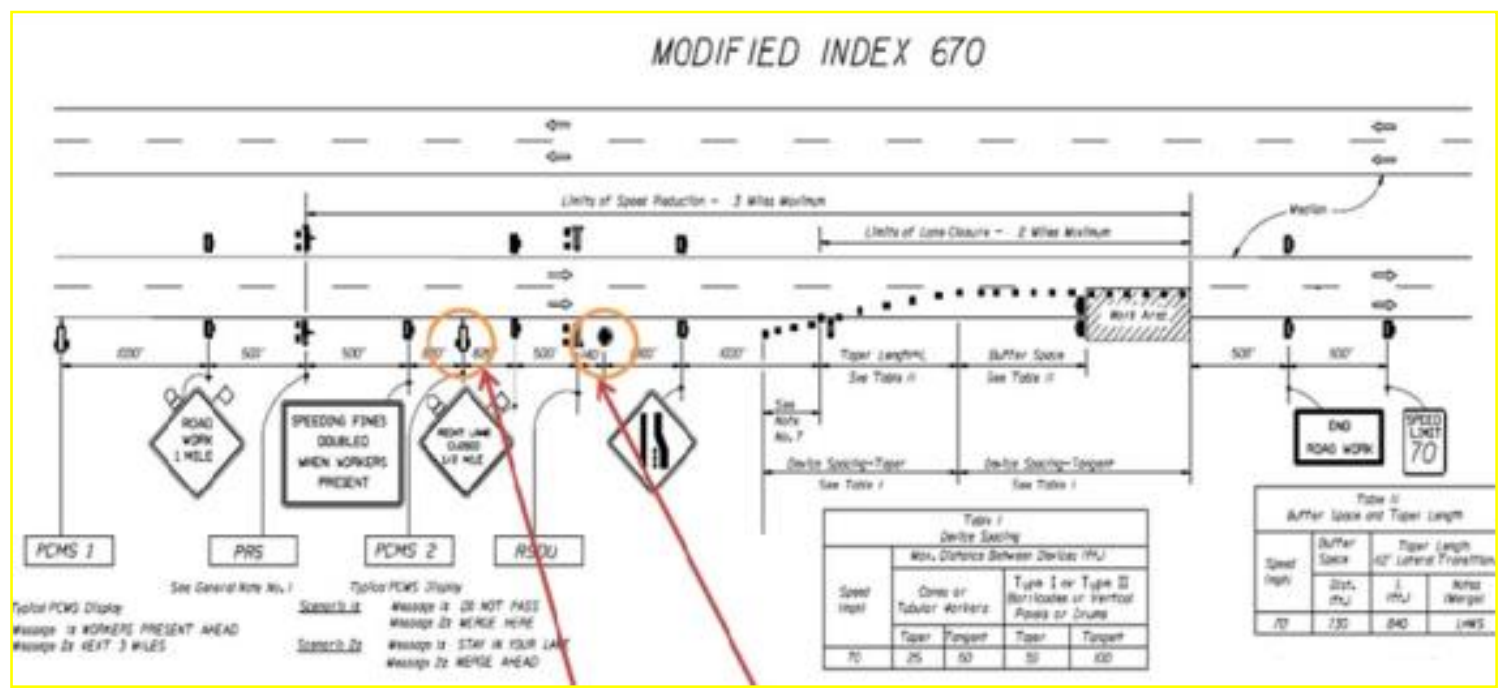

Figure 5 Modified Late Merge Design Suggested by (Redwan et al., 2009)

The suggested layout resulted in an increase from 881 to $970 \mathrm{vph}$ in throughput for shortterm work zone (Redwan et al., 2009). 
The following table summarizes the aforementioned systems in terms of the distance from the taper to the first work zone warning sign, no merge sign "USE BOTH LANES", late merge sign "MERGE HERE", compliance rate and result.

Table 1 Summary of the Distances for Late Merge Systems

\begin{tabular}{|l|l|l|l|l|l|}
\hline System & $\begin{array}{l}\text { Work Zone } \\
\text { Warning }\end{array}$ & No Merge & $\begin{array}{l}\text { Late } \\
\text { Merge }\end{array}$ & $\begin{array}{l}\text { Compliance } \\
\text { Rate }\end{array}$ & Result \\
\hline $\begin{array}{l}\text { Pennsylvania } \\
\text { DOT }\end{array}$ & 2 miles & 1.5 miles & 350 feet & N/A & $\begin{array}{l}\text { Increased } \\
\text { throughput } \\
\text { from 1460 } \\
\text { to } 1540 \\
\text { pcph }\end{array}$ \\
\hline $\begin{array}{l}\text { Minnesota } \\
\text { DOT }\end{array}$ & 2 miles & 1.5 miles & 350 feet & $\begin{array}{l}30-40 \% \text { on- } \\
\text { peak } \\
60 \% \text { off- } \\
\text { peak }\end{array}$ & $\begin{array}{l}\text { Increased } \\
\text { throughput } \\
\text { from 1500 } \\
\text { to 1560 vph }\end{array}$ \\
\hline Maryland DOT & 3 miles & 2 miles & 500 feet & N/A & $\begin{array}{l}\text { Increased } \\
\text { throughput } \\
\text { from 1505 } \\
\text { to 1718 vph }\end{array}$ \\
\hline Michigan DOT & 3 miles & 2 miles & 500 feet & $55-65 \%$ & $\begin{array}{l}\text { Increased } \\
\text { throughput } \\
\text { from 990 to } \\
1207 \text { vph }\end{array}$ \\
\hline Florida DOT & 3 miles & 1.5 miles & 1000 feet & N/A & $\begin{array}{l}\text { Increased } \\
\text { throughput } \\
\text { from 881 to } \\
970 \text { vph }\end{array}$ \\
\hline
\end{tabular}




\subsection{Lane - Changing Models}

Lane changing model is a model that mathematically describe the decision and process of changing a lane along the freeway. Over the last two decades, researchers developed different models assuming logical driver behavior. A few studies have modeled the merging behavior in work zone to determine the desired merging location as well as the probability that drivers succeed in completing the merging maneuver. Most of the merging probability models are based on gap acceptance models describing when the leading and following gaps are accepted by the driver of the subject vehicle (Yang and Koutsopoulos, 1996 and Ahmed, 1999). Moreover, Kita (1999) estimated merging probability based on game theory model. Other studies considered utilizing rule-based model and minimum space gap criterion in order to decide if the current gap is desired or not (Chowdhuty et al., 1997; Nassab et al., 2005; Hidas 2002, 2005). With the emerging of technology in vehicles, Talebpour et al. (2015) presented a lane-changing model that considers the flow of information of connected vehicles using game-theory approach. Gipps (1986) found a model that considers the importunity of the lane-changing maneuver depending on the breaking behavior and driver's gap acceptance. The model is based only on the availability a safe gap with a certain size in the target lane. Gipps' model has been widely utilized in numerous researches because the feasibility of lanechanging is based on relatively simple conditions. Gipps's model is given by the following equation: 
$v_{n}(t+T)=b_{n} T+\left[b_{n}^{2} T^{2}-b_{n}\left(2\left(x_{n-1}(t)-s_{n-1}-x_{n}(t)\right)-v_{n}(t) T-\frac{v_{n-1}(t)^{2}}{b_{n-1}^{e s t}}\right]^{1 / 2}(2)\right.$

Where:

$v_{n}(t+T)$ is the maximum safe speed;

$b_{n}$ is the maximum braking rate;

$T$ is the time between calculations of speed and position;

$x_{n}(t)$ is the location of the front pumper; and

$b_{n-1}^{e s t}$ is the estimate braking of following vehicle by $n$

For the analysis of bottleneck situations such as lane dropping, Fritzsche (1994) revealed a new microscopic traffic simulation model. However, the lane-changing rules are constant without consideration of cooperative or forced lane-changing behavior. Therefore, Yang and Koutsopoulos (1996) developed more detailed rule-based lanechanging model, specifically, for freeways where the lane change is either mandatory or discretionary. The model was constructed with a probabilistic framework considering lane-changing behavior in case of goals conflict. In case of merging into traffic, the model considers a gap as acceptable only if both of the following and leading gaps are acceptable. However, the study seemed to have a lack of parameter estimation. In an attempt to model lane change decisions with discrete choice modeling approach, Ahmed (1999) performed an extensive study. His work generated a probabilistic model to 
describe lane - changing decisions that considers mandatory and discretionary lane changing as well as the situation of forced merge. In fact, the model was a major contribution as it also incorporates gap acceptance probability. The critical gap functional form he guaranteed is given by the following equation

$$
G_{n}^{c r, g}(t)=\exp \left(X_{n}^{g}(\mathrm{t}) \beta^{g} v_{n}+\varepsilon_{n}^{g}(t)\right)
$$

Where:

$g$ is leading and following gap; $X_{n}^{g}(t)$ and $\beta^{g}$ are variables and corresponding parameters; $v_{n}$ is an individual random term distributed normally; and $\alpha^{g}$ is the parameter of $\mathrm{v}_{\mathrm{n}}+\varepsilon_{\mathrm{n}}^{\mathrm{g}}(\mathrm{t})$, which is a generic random term

Toledo (2003) used also a discrete choice modeling approach to model decision and maneuver of lane-changing model. The model considered using maximum likelihood estimation techniques for calibration and applied four classifications to explain variables in lane changing decisions; neighborhood variables, path and plan variables, network knowledge and experience, and driving style and driving capabilities. Toledo implemented the integrated lane changing model in a simulation platform, as Ahmed (1999) did, and compared the anticipated measurements with the results. 
Hidas (2005) classified the mandatory lane - changing into defined three different classifications of lane changing decisions: free, forced and cooperative lane changes. Forced lane _ changing, is when the driver is forced by a merging vehicle to slow down suddenly, which causes emergency breaking. However, cooperative lane-changing is when a driver slow down to enlarge the gap for the other driver to merge. The interaction in cooperative lane-changing is described by Hidas starts when the subject vehicle indicates its willing to merge into the target lane. Then, the follower vehicle perceives the situation and decides to cooperate by slowing down to create a larger gap. Finally, the subject vehicle notices that the follower vehicle cooperated and as a result the gap enough for to executes the maneuver. He described the minimum leading and following gaps by the following equation:

Hidas applied his lane changing decision model in a simulation software and examined the influence of the lane-changing model. The results showed that the estimated speedflow relationship by his model was close to the curve estimated by the Highway Capacity Manual (HCM) 1994.

\subsection{Connected Vehicles Applications in Work Zone}

Intelligent Transportation System (ITS) is a set of information and communication technologies that are applied in the field. Changeable Message Signs (CMS) was one of the early use of ITS in work zone. Dynamic late merge system requires several CMS and 
other ITS devices such as sensors and roadside units. Recently, researchers have paid strong attention to Connected Vehicles (CV) and Autonomous Vehicles (AV) and its applications on freeway as the most advanced ITS technology that can potentially improve mobility and safety of transportation network. ( Uhlemann, 2015).

Federal Highway Administration (FHWA) identified several CV's applications for further research such as Stop Sign Gap Assist (SSGA), Curve Speed Warning (CSW) and Reduced Speed Zone Warning (RSZW). The technology utilizes Dedicated Short-Range Communication (DSRC) to connect Vehicles-to-Vehicle and (V2V) and Vehicle-toInfrastructure (V2I) to exchange data (Chang et al., 2015).

Maitipe (2011) tested using V2V and V2I in work zone by disseminating traffic information such as locations of vehicle congestion and travel times. The proposed system utilizes roadside units in the work zone to share information with vehicles. The field study proved the ability of DSRC to inform CV drivers about the traffic conditions. Davis, L. (2016) went further by demonstrating how CV without any roadside unit can improve flow at a bottleneck. The system depended on Cooperative Adaptive Cruise Control (CACC) to mitigate self-organized congestion. The simulations results proved that $\mathrm{CV}$ can reduce congestion at bottleneck caused by a lane drop of two-lane freeway, even in the case of low market penetration rate.

Wade and Razavi (2016) evaluated the potential benefits of deploying CV on a traffic network in the presence of a work zone in terms of safety. The results revealed that network safety is correlated with the behavior model used. Also, when the market 
penetration rate of $\mathrm{CV}$ was under $40 \%$, the traffic network was safer, while market penetration rate above $40 \%$ reduc es the network safety. Abdulsattar et al. (2017) also assessed the impacts of CV on rear - end collisions in work zone using an agent-based modeling approach. The main preliminary results demonstrated that CV would reduce this type of collision in work zone and the relationship between safety benefits and market penetration rate is nonlinear. 


\section{CHAPTER III}

\section{METHODOLOGY}

This thesis aims to utilize CV technology in work zones and apply a dynamic late merge system in order to improve the capacity of the freeway section. The cooperation concept and its application in the dynamic late merge system with different phases and different scenarios was the key element in this paper. The proposed configuration has been designed with consideration of the nature of work zone merging process as well as the layouts of several states' DOT and their capacity and safety results. Then, the carfollowing and lane-changing models have been selected and modified using $\mathrm{C}++$. Different variables and parameters have been considered to test their influence on the system in terms of capacity, delay and queue length. With all that in mind, the experiment has been designed with 62,100 simulation runs to gain adequate results that would lead to a sufficient analysis of the system.

\subsection{Cooperation Late Merge System (CLMS)}

In this thesis, a cooperative late merge system (CLMS) is proposed and developed. The CLMS is designed based on 3 levels. The three levels reflect an increasing level of connectivity and vehicle automation (see figure 6). The first level depends on V2V technology with the aid of a roadside unit that broadcasts information about the road condition. CVs in the first level negotiate between each other and instruct drivers to apply 
cooperative late merge. In the second level, the roadside unit is upgraded to be a control unit that communicates with CVs and receives information from them in order to instruct them to apply cooperative merge. The third level is based on adding AV to the second stage. The following graph describes the overall idea and major components of CLMS in its three stages: 


\section{Level 1: Decentralized Cooperative Merge}

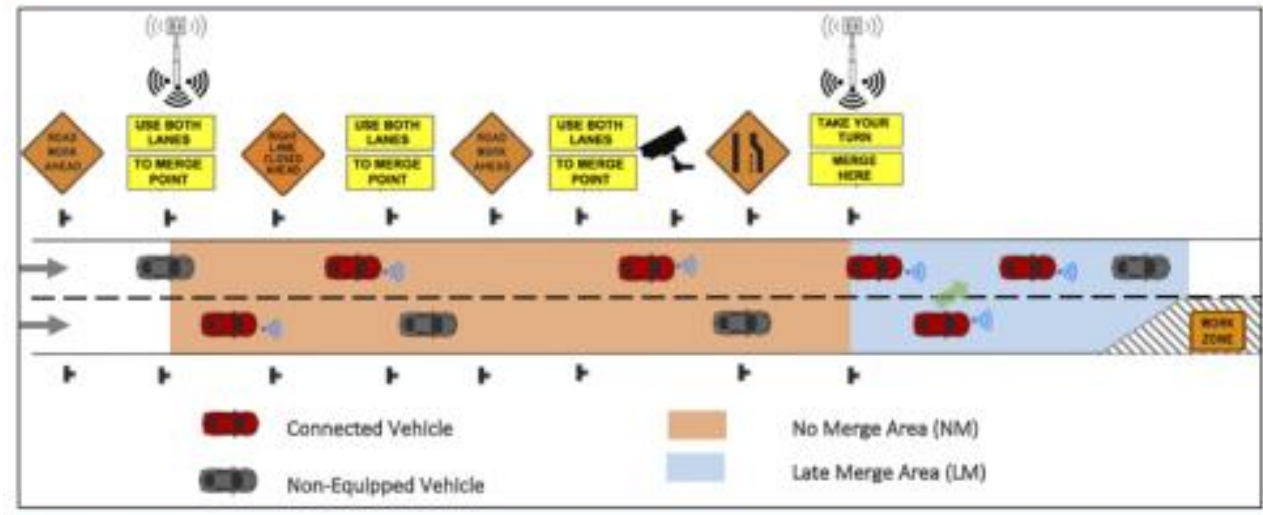

Level 2: Centralized Cooperative Merge

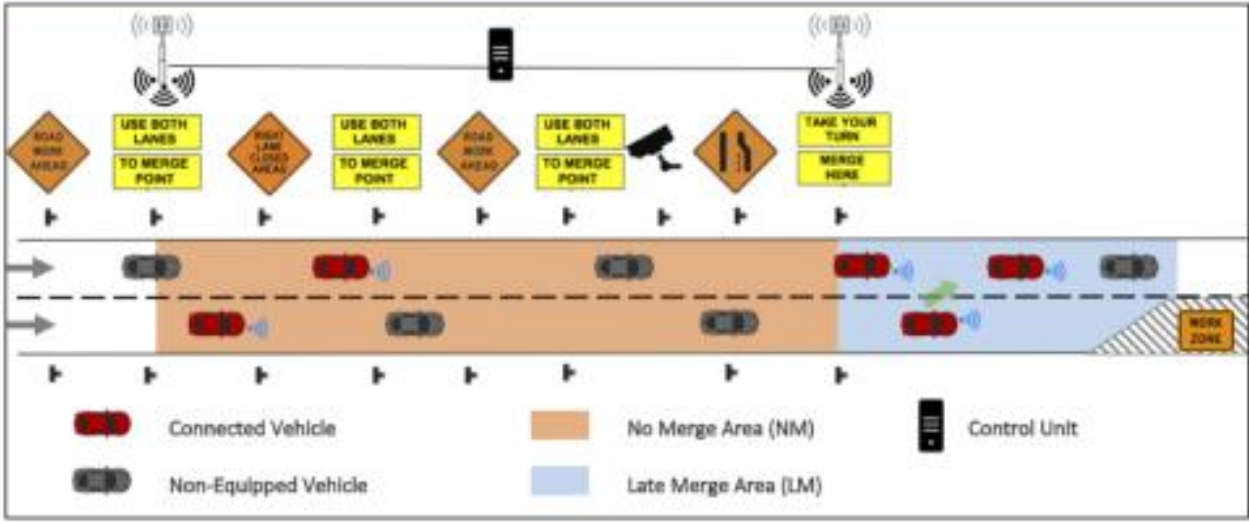

Level 3: Centralized Cooperative Merge (CV + AV)

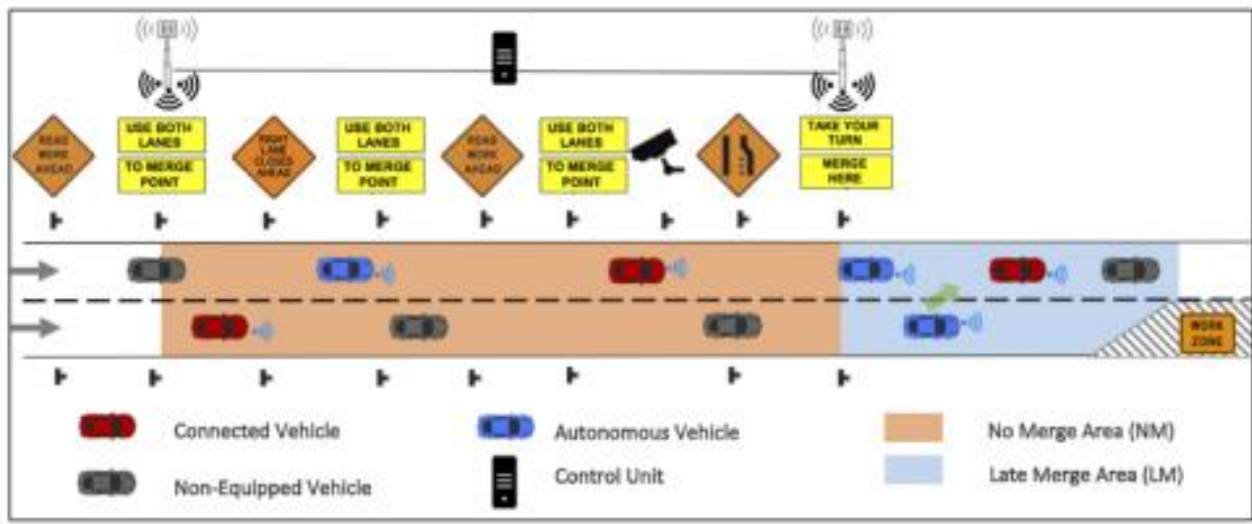

Figure 6 Description of Three Stages of CLMS

The capacity of work zone is influenced by the number of lanes on the freeway and the number of dropped lanes. Due to the complexity of considering several number of lanes 
and multiple dropped lanes, the CLMS in this thesis will focus on two-lane freeway with one dropped lane. Also, the thesis will concentrate only on Level 1 of CLMS in order to conduct a deep analysis of the system performance.

CLMS Level 1 consists of No Merge Area and Late Merge Area with different dimensions, purposes and components. No Merge Area has a broadcast unit that notifies $\mathrm{CV}$ about staying in their lanes and not to merge as well as a series of MUTCD signs. Late Merge Area also has a roadside unit, but to notify CV about starting merging area. In this area, $\mathrm{CV}$ will connect and share information to apply cooperative merge. The components and control algorithm of Level 1 CLMS is described in the following sections.

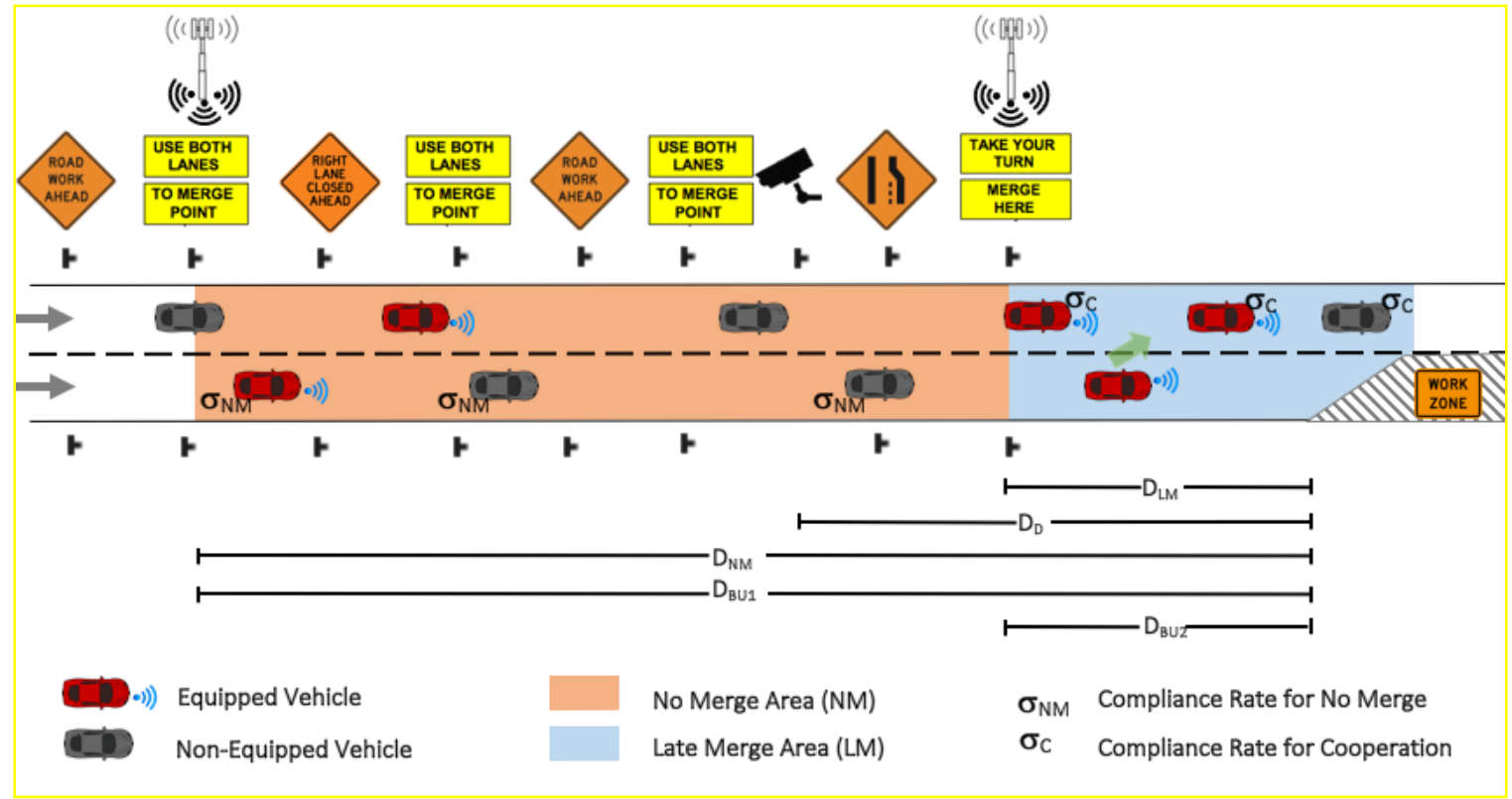

Figure 7 Major components of Level 1 CLMS 


\subsection{Traditional Work Zone}

In traditional work zone, a series of signs are placed to warn drivers about work zone and lane closure. The main goal is to ensure that drivers are aware of lane dropping to reduce speed and allow merging for vehicles in the closed lane. However, the system is not specifically designed to improve the capacity of work zone and reduce the overall delay of vehicles. Most of DOTs use the traditional work zone layout suggested by MUTCD, which gives the drivers the choice to merge at any position before the taper. Traditional Work Zone will be considered in the simulation. In performance analysis, CLMS will be compared with the traditional work zone.

\subsection{Late Merge System}

Late merge is an increasingly common practice by different DOTs as it is found to improve the capacity of work zone comparing to traditional work zone. However, the relatively low compliance rate by drivers limit the benefit of the system. The late merge system consists of a series of signs that instruct drivers to use both lanes and merge downstream closer to the taper. It mainly depends on the signs and drivers' compliance with the instructions to implement the system. In this study, the performance of CLMS will be compared with late merge system as well. 


\subsection{CLMS Level 1: Decentralized Cooperative Merge}

Common late merge systems rely on drivers' understanding of signs and their compliance with signs' instructions, which, in the field, has shown limited benefits. Taking advantage of CVs can enhance the system by notifying drivers about signs' instructions and guide them via Driver Vehicle Interface (DVI) to cooperate with other vehicles to merge. The designed system is called decentralized, where agents in CVs negotiate merging decisions and processes among each other. The CLMS-Level 1 relies on roadside units (Broadcasting Units) that notify CVs about the beginning of No Merge Area and Late Merge Area. It also counts on the capability of V2V communication to exchange driving information in order to increase the chance of cooperative merge.

CLMS-Level 1 is an agent-based system with each vehicle being represented by a computer agent that communicates with other vehicles through DSRC communications. The agent will process information received and follow the CLMS level-1 rules. It will then provide drivers merging suggestions. If the vehicle is driving in the open lane, it will be suggested to cooperate and yield for the car in the closed lane. On the other hand, if the vehicle is driving in the closed lane, it will be suggested to merge when the vehicle in the open lane is willing to cooperate. The driver will choose to take the suggestion or ignore it according to his/her own desire. This factor is described by a compliance rate in CLMS Level 1. The following flowchart describes the logic of CLMS in work zone area for both $\mathrm{CV}$ and non-CV: 


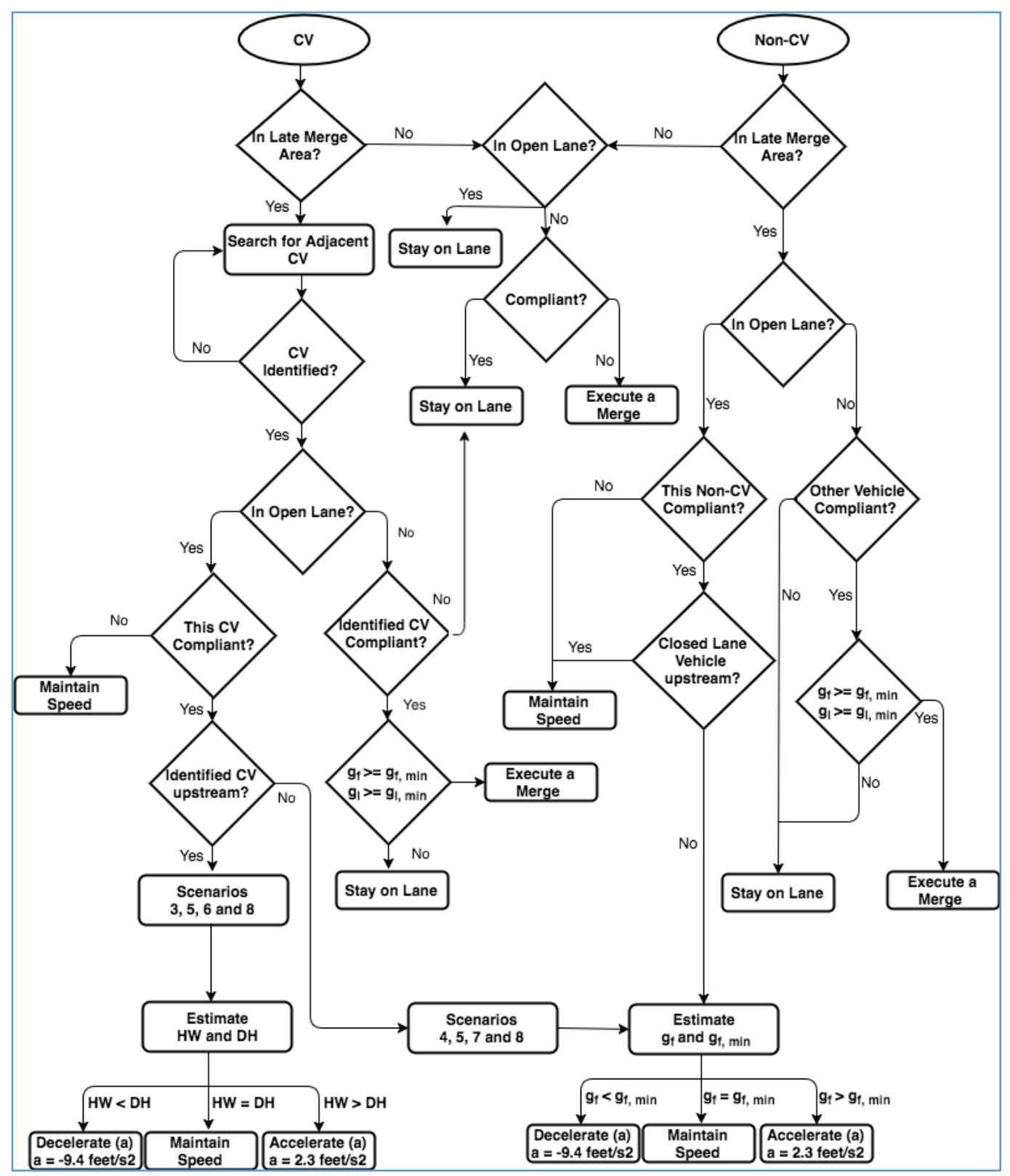

Figure 8 Flowchart of the Logic of CLMS in Work Zone Area

Along the freeway, drivers are free to change their lanes at any time as long as there is no sign to restrict them. However, in the no merge area before work zone, drivers are asked to stay in their lanes without lane-changing and to merge later close to the taper. Then, in late merge area, drivers are asked to cooperate for merging. 
The system aims to enhance cooperation between CVs in order to apply late merge and improve work zone capacity. When CVs are instructed to apply late merge through broadcasting units on the roadside, the CVs' agents can negotiate the merging process for the work zone capacity interest. By utilizing Dedicated Short-Range Communication (DSRC), CVs can exchange travel information such as location, speed and acceleration. When a $\mathrm{CV}$ in the open lane detected the existence of another $\mathrm{CV}$ in the closed lane of the late merge area, it will notify the driver to reduce or increase the speed. The CV in the closed lane will notify the driver to merge in the gap created by the CV in the open lane.

With consideration of various possible interactions between merging, following, and leading vehicles in the open and closed lanes, the algorithm of the level 1 CLMS is composed of the following mutually exclusive and collectively exhaustive scenarios.

\subsubsection{Cooperative Merge Scenarios in CLMS}

The location of $\mathrm{CVs}$ in the late merge area, which varies from one scenario to another, is the key element of the system. The market penetration rate plays a significant role in the occurrence of certain scenarios. For example, a low market penetration rate would increase the probability of having a scenario of a $\mathrm{CV}$ being surrounded by non-equipped vehicles. However, a high market penetration rate would increase the probability of having a scenario of a $\mathrm{CV}$ being surrounded by $\mathrm{CVs}$. The following table summarizes the different scenarios considered in this system: 
Table 2 Summary of Scenarios Considered in CLMS

\begin{tabular}{|c|c|c|c|c|c|c|}
\hline Scenario & $\begin{array}{l}\text { Number } \\
\text { of CVs in } \\
\text { Open } \\
\text { Lane }\end{array}$ & $\begin{array}{l}\text { Number } \\
\text { of CVs } \\
\text { in } \\
\text { Closed } \\
\text { Lane }\end{array}$ & $\begin{array}{l}\text { Subject } \\
\text { Vehicle }\end{array}$ & $\begin{array}{l}\text { Following } \\
\text { Vehicle }\end{array}$ & $\begin{array}{l}\text { Leading } \\
\text { Vehicle }\end{array}$ & Description \\
\hline 1 & 0 & 0 & Non-CV & $\begin{array}{l}\text { Non- } \\
\mathrm{CV} / \mathrm{CV}\end{array}$ & $\begin{array}{l}\text { Non- } \\
\mathrm{CV} / \mathrm{CV}\end{array}$ & $\begin{array}{l}\text { No } \\
\text { Communication } \\
\text { between } \\
\text { vehicles and } \\
\text { merging } \\
\text { depends on } \\
\text { driver courtesy }\end{array}$ \\
\hline 2 & 0 & 1 & $\mathrm{CV}$ & Non-CV & Non-CV & $\begin{array}{l}\text { No } \\
\text { Communication } \\
\text { between } \\
\text { vehicles and } \\
\text { merging } \\
\text { depends on } \\
\text { driver courtesy }\end{array}$ \\
\hline 3 & 1 & 1 & $\mathrm{CV}$ & $\mathrm{CV}$ & Non-CV & $\begin{array}{l}\text { Following } \\
\text { vehicle } \\
\text { instructed to } \\
\text { cooperate by } \\
\text { slowing down }\end{array}$ \\
\hline 4 & 1 & 1 & $\mathrm{CV}$ & Non-CV & $\mathrm{CV}$ & $\begin{array}{l}\text { Leading vehicle } \\
\text { instructed to } \\
\text { cooperate by } \\
\text { speeding up }\end{array}$ \\
\hline 5 & 2 & 1 & $\mathrm{CV}$ & $\mathrm{CV}$ & $\mathrm{CV}$ & $\begin{array}{l}\text { Mix of both } \\
\text { scenario } 3 \text { and } \\
4\end{array}$ \\
\hline 6 & 1 & 2 & $\mathrm{CV}$ & $\mathrm{CV}$ & $\mathrm{CV}$ & $\begin{array}{l}\text { Following } \\
\text { vehicle } \\
\text { instructed to } \\
\text { cooperate by } \\
\text { slowing down } \\
\text { to let both CVs } \\
\text { merge }\end{array}$ \\
\hline
\end{tabular}




\begin{tabular}{|l|l|l|l|l|l|l|}
\hline 7 & 1 & 2 & CV & CV & CV & $\begin{array}{l}\text { Following } \\
\text { vehicle } \\
\text { instructed to } \\
\text { cooperate by } \\
\text { slowing down } \\
\text { to let both CVs } \\
\text { merge }\end{array}$ \\
\hline 8 & 2 & 2 & $2 \mathrm{CVs}$ & $\mathrm{CV}$ & $\mathrm{CV}$ & $\begin{array}{l}\text { Mix of both } \\
\text { scenario } 6 \text { and } \\
7\end{array}$ \\
\hline
\end{tabular}

\subsubsection{Scenario 1}

There is a chance that Late Merge Area has only non-equipped vehicles, which reduces the probability of cooperative merge. This scenario is similar to the conventional late merge system, which relies on drivers' compliance and courtesy in helping vehicles in the closed lane to complete merging process. In this case, non-equipped vehicles follow normal car-following and lane-changing model and their cooperation depends on their compliance with the system. Since drivers in the open lane are asked to give other drivers in the open lane the chance to merge by using the sign "TAKE YOUR TURN", there is a chance of cooperative merge by non-equipped vehicles as it was observed by several studies for typical late merge system. Therefore, the compliance rate will decide the ratio of non-equipped vehicles that will show courtesy to other drivers and yield for merging to be completed. However, the reaction of the driver of the non-equipped vehicle in the closed lane can be slower compared with $\mathrm{CV}$ because he/she requires an adequate gap encouraging him/her to make a merging decision. 


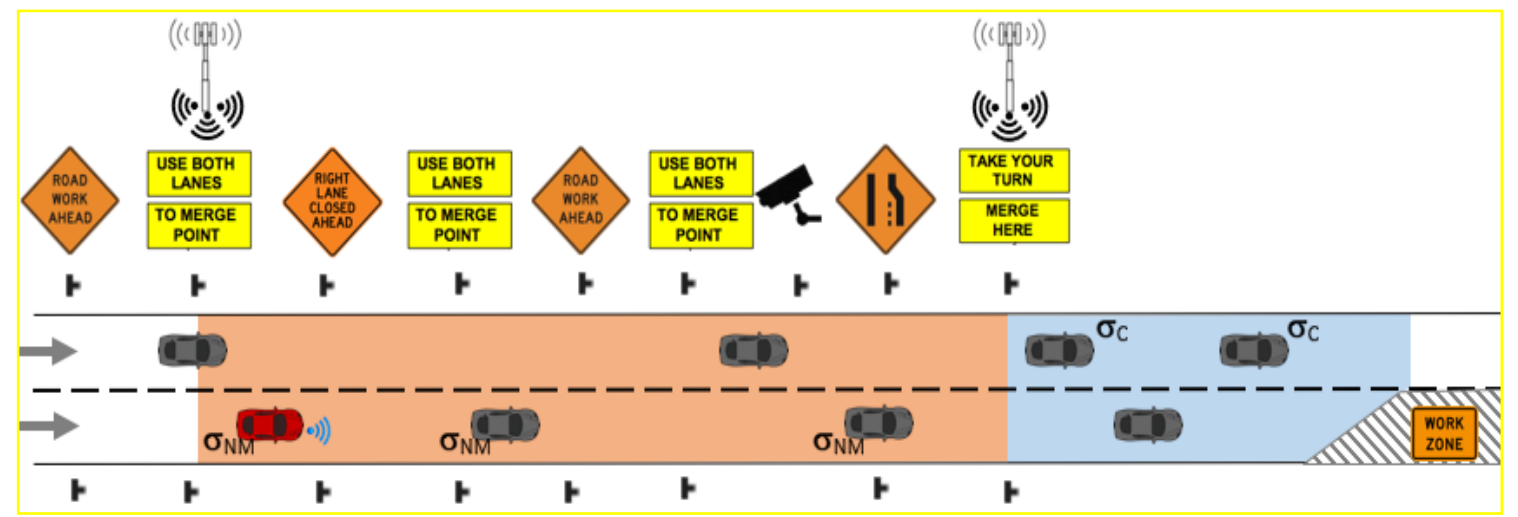

Figure 9 Scenario 1 in CLMS

\subsubsection{Scenario 2}

In this scenario, $\mathrm{CV}$ in the closed lane is surrounded by non-equipped vehicles, which makes it unable to communicate with surrounded vehicles. This restricts cooperative merge and makes merging probability dependent only on the courtesy of non-equipped vehicles in the open lane. In fact, this scenario is similar to Scenario 1 as CV in this case will act as non-equipped vehicle.

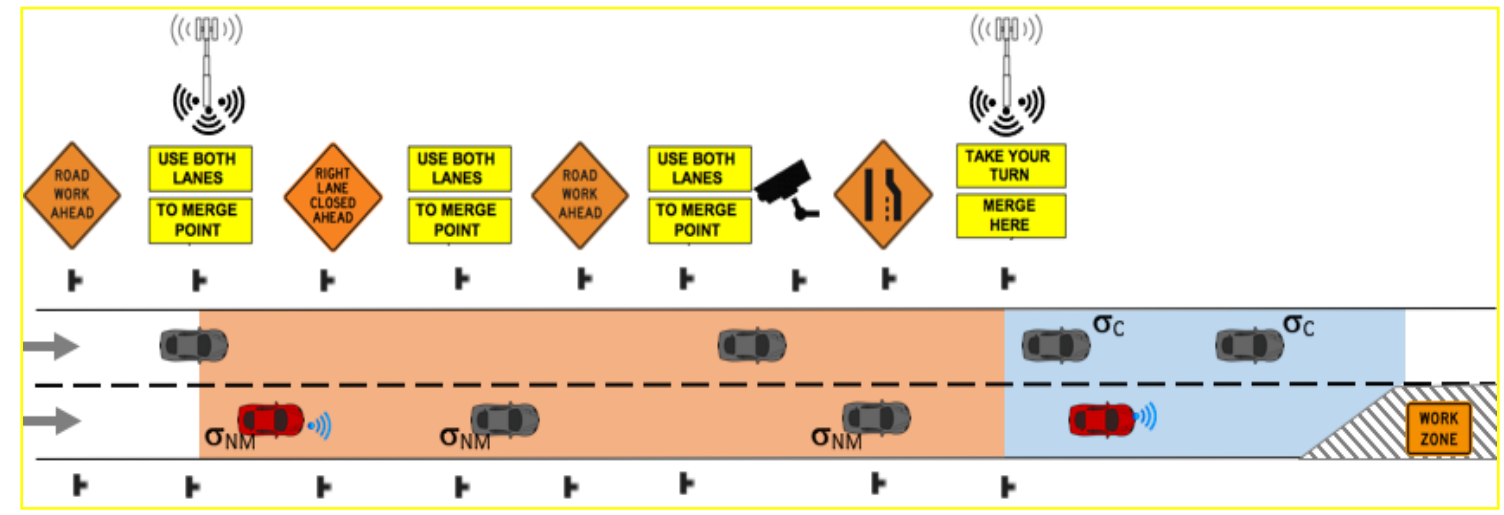

Figure 10 Scenario 2 in CLMS 


\subsubsection{Scenario 3}

In this scenario, $\mathrm{CV}$ in the open lane is able to communicate with the following vehicle, which makes merging probability higher. The following vehicle can reduce the speed to increase the gap and allow the subject vehicle in the open lane to complete the merge as appear in Figure 10. Technically, the agent of the CV in the closed lane requests merging from the agent of the $\mathrm{CV}$ in the open lane, providing it with its speed, location and acceleration. The agent of the $\mathrm{CV}$ in the open lane calculates the required speed reduction that would result from the merging process. If it is feasible, the driver is notified to reduce speed to a certain value and if the driver complied and started decelerating, the agent sends merging confirmation to the agent of the vehicle in the closed lane (downstream) to complete merging process if it is possible.

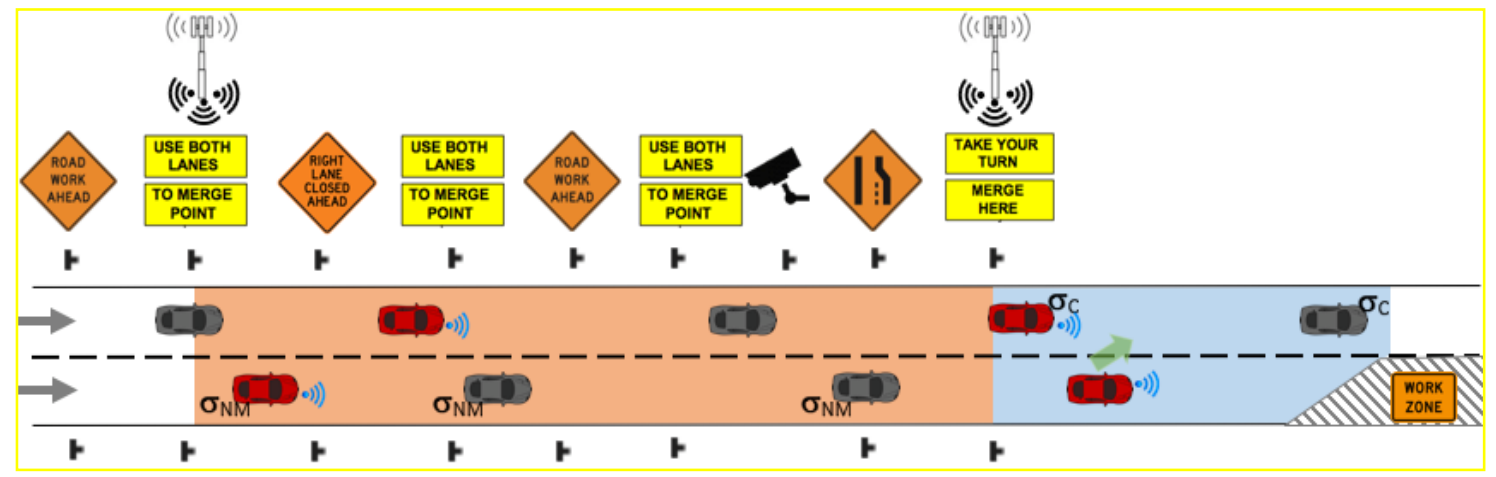

Figure 11 Scenario 3 in CLMS

In order to apply cooperative merge in such a case, Downstream Flowchart is created as the following: 


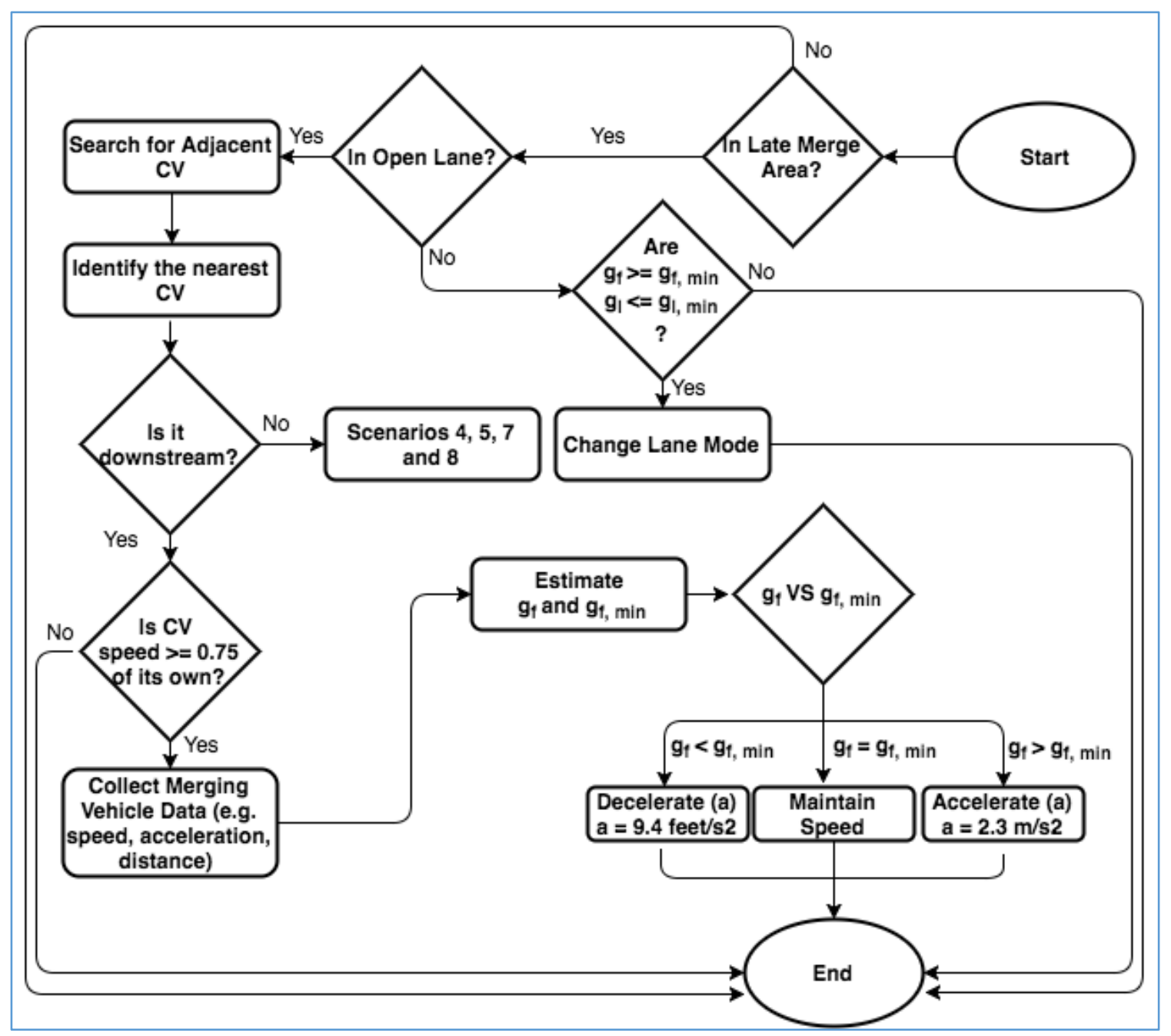

Figure 12 Flowchart of the CLMS Logic in Downstream Scenarios

The communication between CVs starts when the CV in the open lane of Late Merge Area searches for adjacent CV in the closed lane. When a CV is identified, the agent recognizes whether it is downstream or upstream. If it is downstream, it will follow Downstream Flowchart, while if it is upstream, then it will follow Upstream Flowchart. In Downstream Flowchart, if the speed of the CV in the closed lane is lower than $75 \%$ of the $\mathrm{CV}$ in the open lane, the communication is neglected and CV in the open lane starts searching for another $\mathrm{CV}$. If the speed is equal to or higher than $75 \%$, the $\mathrm{CV}$ in the open 
lane collects data from CV in the closed lane including speed, acceleration and distance. It then estimates the current following gap and the minimum following gap for merging. If the current following gap equals minimum following gap, $\mathrm{CV}$ in the open lane maintains its speed. If the current following gap is smaller than minimum following gap, the $\mathrm{CV}$ in the open lane decelerates to enlarge the gap, while if it is larger, it accelerates to reduce the gap. The $\mathrm{CV}$ in the closed lane inspects the current following and leading gap. If they both equal or larger than the minimum, it starts lane changing mode.

\subsubsection{Scenario 4}

In this scenario, the $\mathrm{CV}$ in the open lane is a leading vehicle and the possible solution is to increase the speed to enlarge the gap for the $\mathrm{CV}$ in the open lane to complete the merge (see Figure 12). This scenario would be restricted by the available gap between the leading vehicle and vehicle in front of it. In fact, the agent of the CV in the closed lane requests merging from the agent of the $\mathrm{CV}$ in the open lane (upstream) and the agent of the $\mathrm{CV}$ in the open lane asks the driver to increase the speed to the speed limit if it is possible. If the driver complied and started accelerating, the agent sends merging confirmation to the agent of the vehicle in the closed lane in order to complete merging process if it possible. 


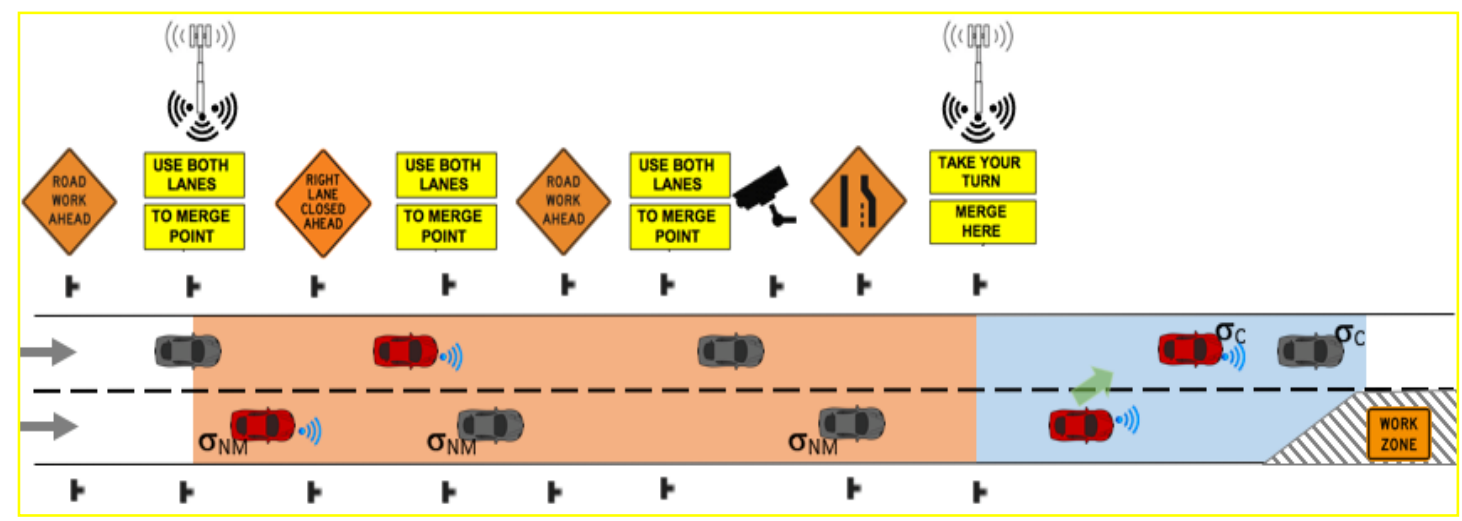

Figure 13 Scenario 4 in CLMS

Upstream Flowchart describes the process of applying cooperative merge as the following:

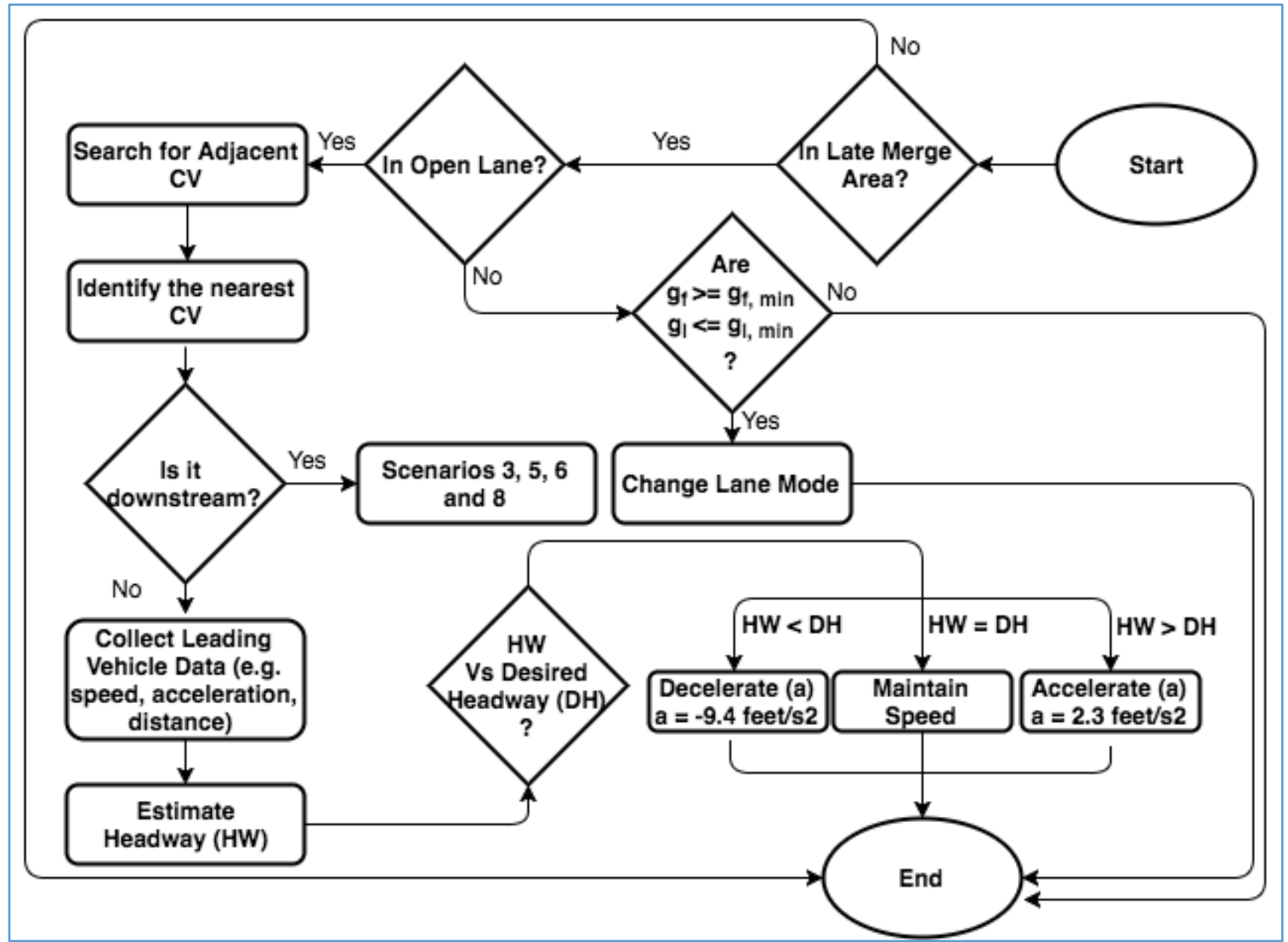

Figure 14 Flowchart of the CLMS Logic in Upstream Scenarios 
In Upstream Flowchart, the CV in the open lane collects data from leading vehicle. It then estimates the headway (HW). If the HW equals to the Desired Headway (DH), CV in the open lane maintains its speed. If $\mathrm{HW}$ is smaller than $\mathrm{DH}$, the $\mathrm{CV}$ in the open lane decelerates to have a safe gap with the leading vehicle, while if it is larger, it accelerates to enlarge the gap. The CV in the closed lane inspects the current following and leading gap. If they both equal to, or larger than the minimum, it starts lane changing mode.

\subsubsection{Scenario 5}

In this scenario, $\mathrm{CV}$ in the open lane is surrounded by leading and following CVs. This case would make it easier to enlarge the gap as the following vehicle can reduce its speed and the leading vehicle can increase its speed (see Figure 15). The communication process between the $\mathrm{CV}$ in the open lane and following vehicle follows Downstream Flowchart, while with the leading vehicle it follows Upstream Flowchart. The advantage of this scenario is that if the following or leading driver declines to comply with the $\mathrm{CV}$ order, there is a chance that the other would cooperate to help completing the merging process. 


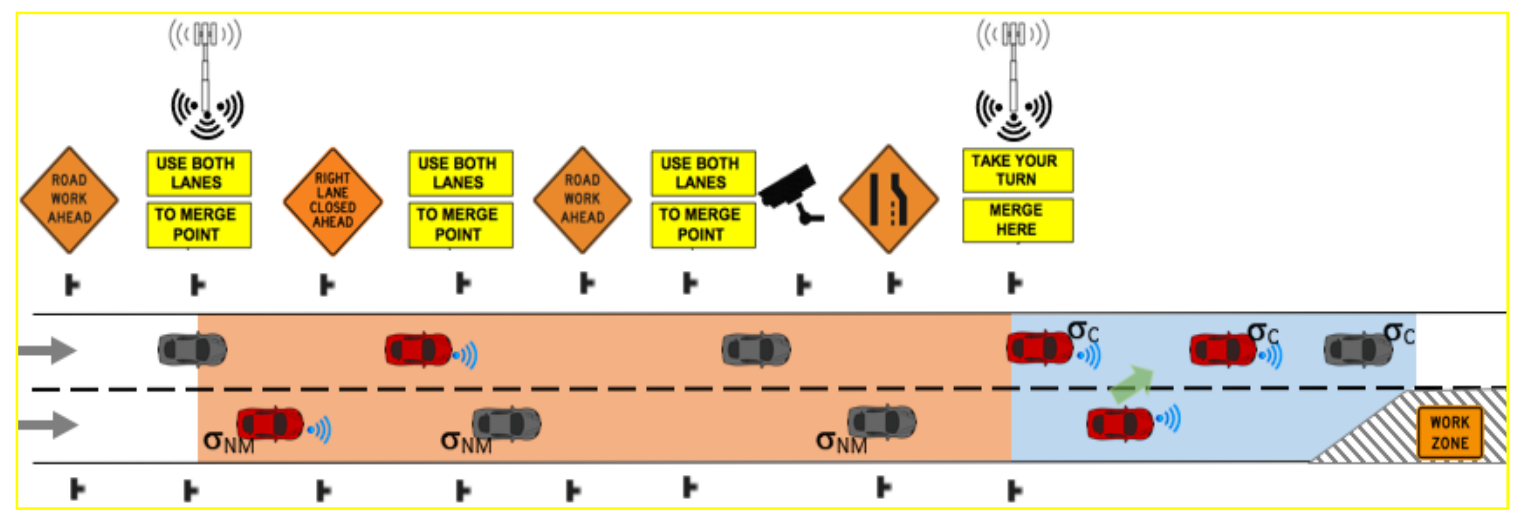

Figure 15 Scenario 5 in CLMS

\subsubsection{Scenario 6}

When there is only one following CV in the open lane and two CVs in the closed lane, the $\mathrm{CV}$ in the open lane can reduce the speed to increase the gap for the nearest subject vehicles to complete the merge (see Figure 16). The communication process between agents follows Downstream Flowchart. The second CVs in the open lane would also have the chance to merge with the first one. If not, the first merged CV can then cooperate and create a gap for the second vehicle in the open lane to merge. 


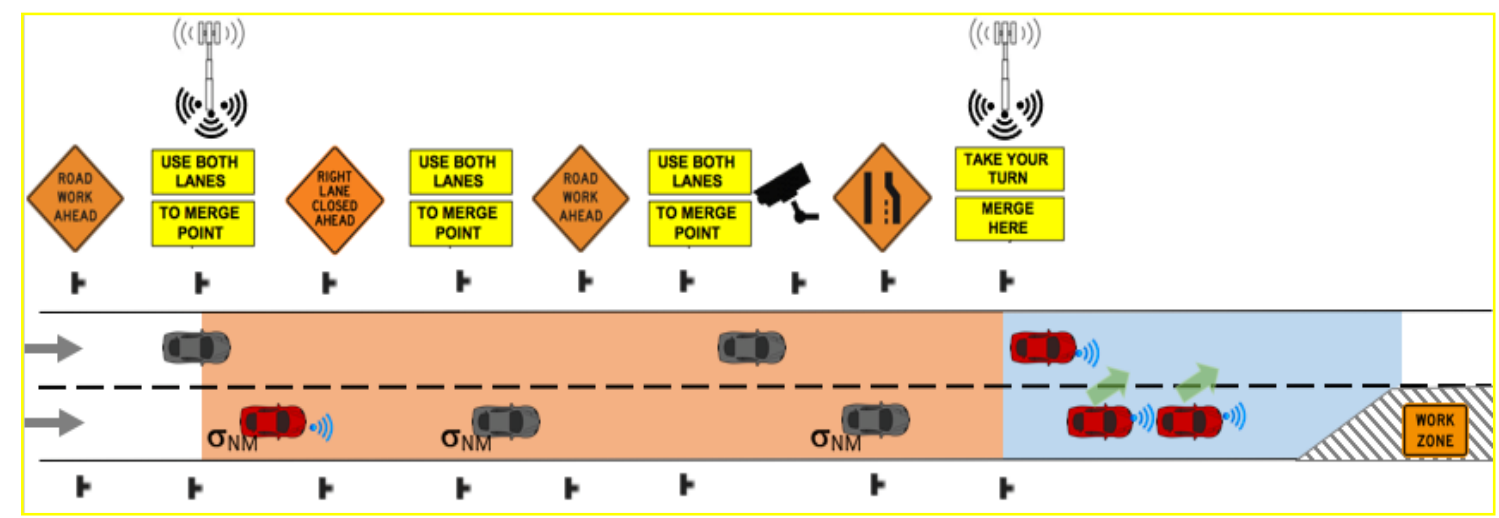

Figure 16 Scenario 6 in CLMS

\subsubsection{Scenario 7}

In this scenario, there is one leading CV in the open lane and two CVs in the closed lane (see Figure 17). The CV in the open lane can increase the speed to increase the gap for the nearest $\mathrm{CV}$ in the open lane to complete the merge. The communication process between agents follows Upstream Flowchart. The second CVs in the open lane would also have the chance to merge with the first one. If not, the first merged CV can then cooperate and create a gap for the second vehicle in the open lane to merge.

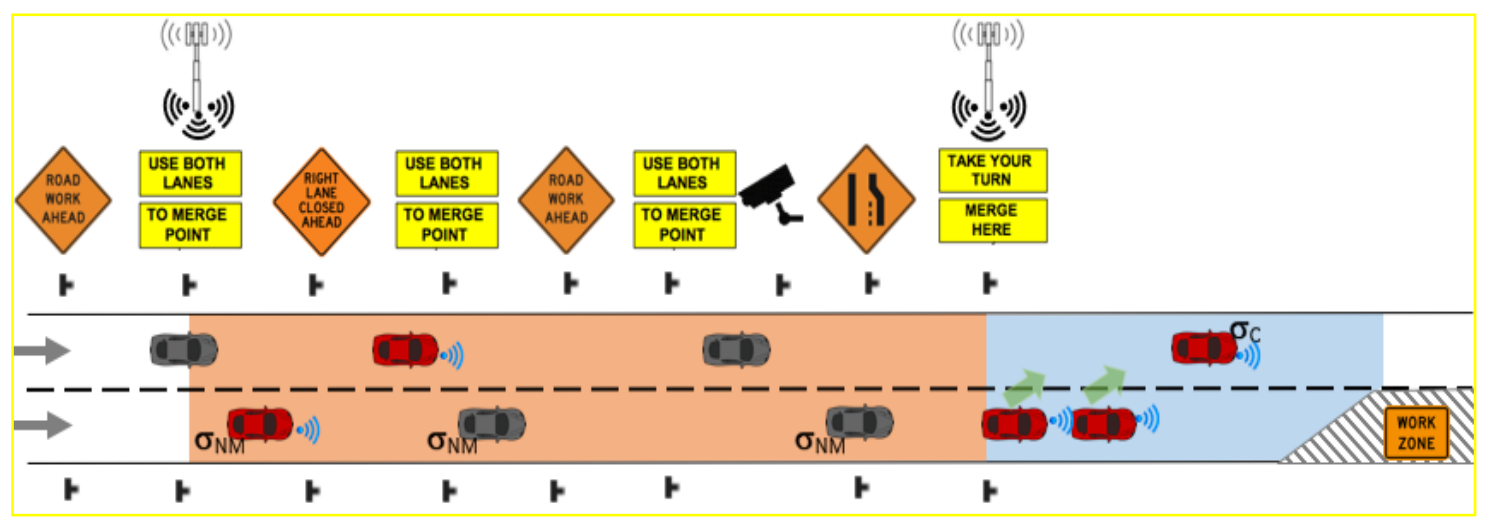

Figure 17 Scenario 7 in CLMS 


\subsubsection{Scenario 8}

In this scenario, there are two CVs in the open lane and two CVs in the closed lane (see Figure 18). The leading vehicle can increase the speed to enlarge the gap, while the following vehicle can reduce the speed to allow the two $\mathrm{CV}$ s to complete the merge. In this situation, the first $\mathrm{CV}$, which is closer to the taper, has the priority to merge followed by the second CV. This scenario can be achieved by following both Downstream and Upstream Flowchart.

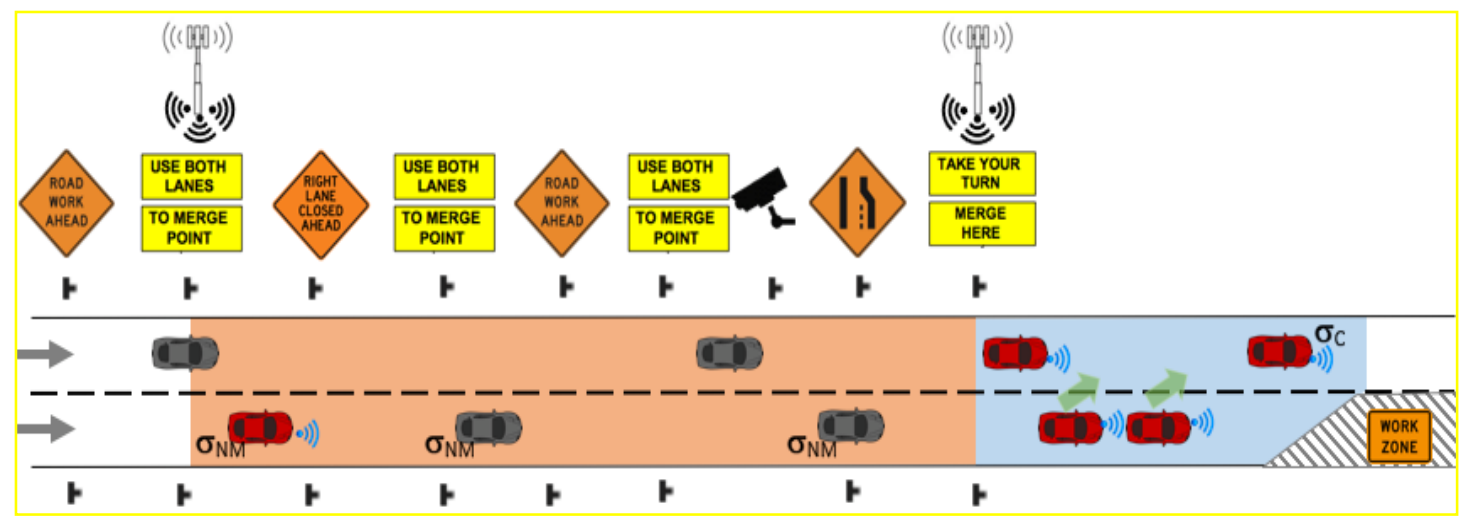

Figure 18 Scenario 8 in CLMS

\subsubsection{System Configuration and Key Components}

The dynamic late merge system is based on several Portable Changeable Message Signs (PCMSs) that is activated when congestion is detected. PCMSs instruct drivers to use both lanes to the merge point when traffic conditions require late merge. Other MUTCD advance warning signs are also statically installed to aware drivers about the work zone. Therefore, it is very important to identify the main components with their appropriate distances. Figure 6 illustrates this dynamic late merge system and its main components. 


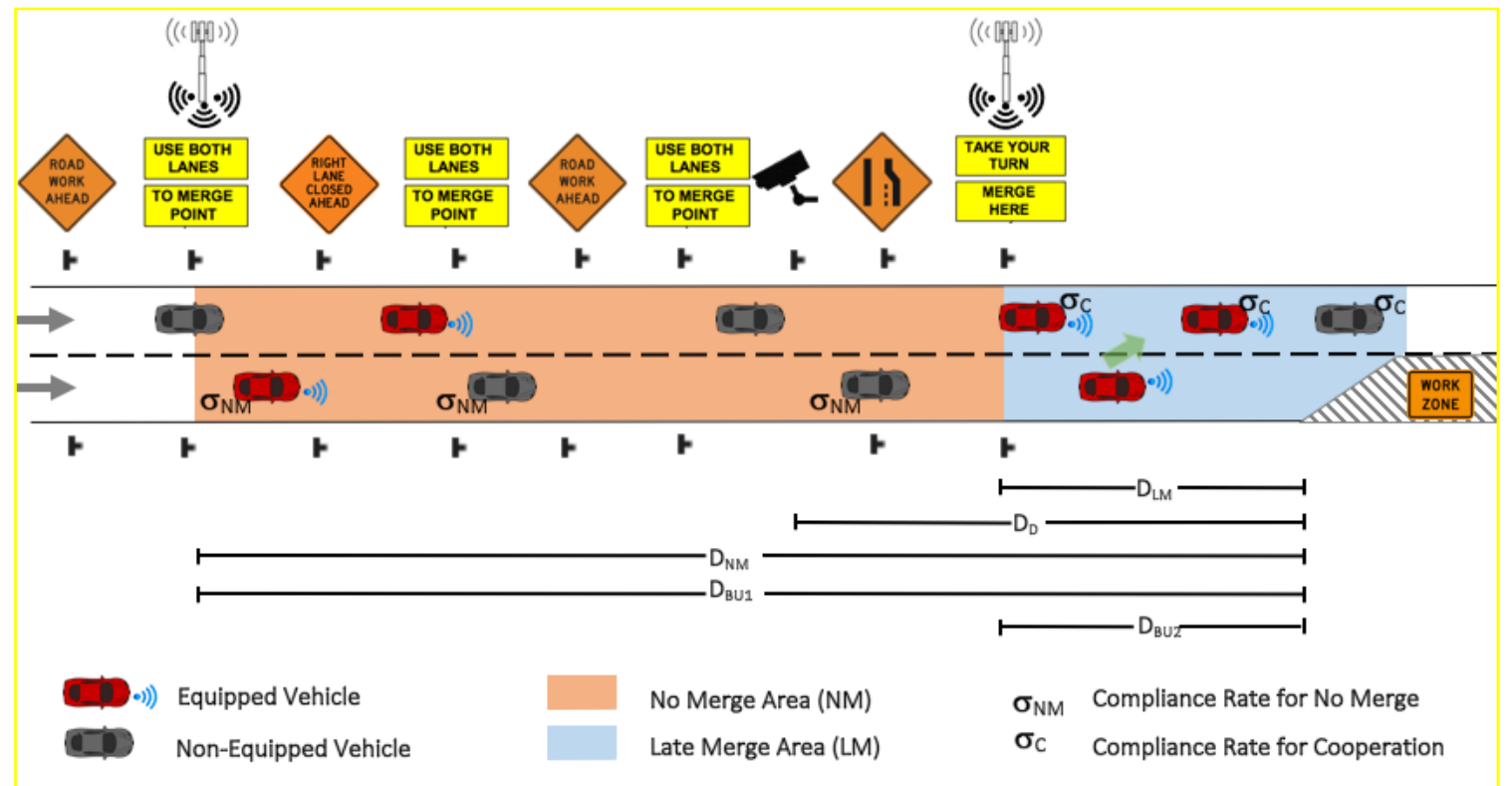

Figure 19 System Configuration and Key Components

The following table describes the distances in CLMS-Level 1:

Table 3 Distances of CLMS Layout

\begin{tabular}{|l|l|}
\hline Distance & Description \\
\hline$D_{N M}$ & Distance of No Merge Area from the taper \\
\hline$D_{L M}$ & Distance of Late Merge Area from the taper \\
\hline$D_{D}$ & Distance of the detector from the taper \\
\hline$D_{B U 1}$ & Distance of the first Broadcasting Unit from the taper \\
\hline$D_{B U 2}$ & Distance of the second Broadcasting Unit from the taper \\
\hline
\end{tabular}

System components have to be located appropriately to ensure that the whole system can work efficiently. As appears in Figure 2, there are several Advance Warning Signs, which are static. All this type of signs is located within the range that suggested by MUTCD. DLM is the distance of Late Merge Area, where vehicles are supposed to eventually merge. $D_{\mathrm{NM}}$ is the distance of No Merge Area, where vehicles are supposed to stay in 
their lanes. It is worth noting that the sign of "USE BOTH LANES" must be placed before the sign that indicates which lane is closed.

$D_{D}$ is the distance from the taper to the detector that measures the traffic volume and speed of vehicles. DBul is the distance of the first Broadcasting Unit from the taper. The function of the first broadcasting unit is to inform CVs about No Merge Area and instruct

drivers to use both lanes. Therefore, it has to be placed somewhere close to the first "USE BOTH LANES" sign and the CV driver should be notified within the sight distance of the sign. $\mathrm{D}_{\mathrm{BU} 2}$ is the distance of the second Broadcasting Unit from the taper. The function of the second broadcasting unit is to inform CVs about Late Merge Area and instruct drivers to cooperate with other drivers to complete merging process. This second broadcasting unit has to be placed somewhere close to "MERGE HERE" sign and the CV driver should be notified within the sight distance of the sign.

\subsubsection{Distance Variables}

The advanced warning area is a section on freeway where drivers are informed about upcoming work zone. As suggested by MUTCD, there are three main advisory signs to be placed before work zone taper. The furthest sign from the taper informs drivers that there is a road work ahead. It is followed by another sign that advises drivers about which lane is closed. Then, a sign is placed closer to the taper advising drivers to merge. MUTCD suggests certain spacing for the distance between the three main advisory signs depending on the road type. 
The system takes into consideration different type of distances as suggested by MUTCD. Specifying the distance for each variable is a key element in the development of a late merge system with an improved throughput. To warn driver about the work zone and to reduce their speed, an advisory sign should be placed at least 1 mile before the taper as suggested in Table 2. As mentioned earlier in Table 2, most of late merge systems start to warn drivers about 2 or 3 miles from the taper. In this system, the first sign "ROAD WORK AHEAD” is placed 2 miles before the taper.

\subsubsection{No Merge Distance $\left(D_{N M}\right)$}

Another advisory sign about which lane is closed should be placed at least 2500 feet from the taper. The location of the closed-lane sign is significant as it is the first notification about which lane vehicles are going to merge to. When vehicles observe the sign, there is a high probability to execute an early merge rather than late merge. Therefore, "USE BOTH LANES” sign has to be placed just before closed lane sign. In this system, "RIGHT LANE CLOSED AHEAD" sign is placed half mile (2640 feet) before the taper. That being said, $\mathrm{D}_{\mathrm{NM}}$ is going to be more than 2640 feet from the taper. Thus, "USE BOTH LANES" sign is placed 400 feet before "RIGHT LANE CLOSED AHEAD" sign, which means DNM equals 3140 feet.

\subsubsection{Late Merge Distance $\left(D_{L M}\right)$}

Studies conducted by different DOTs have considered different late merge distances in their systems as Table 2 shows. Dynamic Late Merge (DLM) System designed by Maryland State Highway Administration allocates the merge point 500 feet away from 
the work zone taper (Kang et al., 2006). However, Pennsylvania DOT and Michigan DOT uses a PCMS that displays "Merge Here/ Take Your Turn" 500 feet prior to the taper (Datta et al, 2008). Other system allocate the merge point at 1000 and 1500 feet from the taper.

The main factor deciding whether to implement early merge or late merge is arriving vehicles rate. When arriving vehicles rate is low, early merge is encouraged and vehicles are free to merge at any point. However, when arriving vehicles rate is moderate and close to the capacity of the freeway, late merge is necessary to improve the capacity of work zone (Grillo, et al, 2008).

In early merge, merging location is various and it is unnecessary to determine a specific point as vehicles are free to merge at any point. However, late merge requires merging close to the taper and as arriving vehicles increases the need to merge closer to the taper increases to exploit more space of the closed lane. Also, the high arriving vehicles means high density and therefore less chance to find merging gap before reaching the taper. Tympakianaki et al. (2014), studies the flow-density relationship for the merge area. When arriving vehicles rate is low, merging conflicts are less and throughput is correspondingly low. As arriving vehicles increase, the chance of merging conflicts is very high, but throughput may increase as well until reaching critical arriving vehicles, where throughput attains the downstream capacity. Therefore, throughput $\left(\mathrm{V}_{\mathrm{T}}\right)$ is inversely proportional to arriving vehicles $\left(\mathrm{V}_{\mathrm{A}}\right)$ and the equation as the following:

$$
V_{T}=\frac{V_{A}}{a}
$$


Where:

$V_{T}$ is vehicles throughput;

$V_{A}$ is number of arriving vehicles; and

$a$ is a variable

The expected curve in the relation between $\mathrm{V}_{\mathrm{A}}$ and $\mathrm{V}_{\mathrm{T}}$ follows a negative polynomial function as the following:

$$
f(x)=a x^{2}+b x+c
$$

Where:

$f(x)$ is the throughput $\left(\mathrm{V}_{\mathrm{T}}\right)$;

$x$ is the number of arriving vehicles $\left(\mathrm{V}_{\mathrm{A}}\right)$; and

$a, b$ and $c$ are constants.

Several studies recommend applying late merge in moderate traffic volume. Minnesota DOT recommends that late merge is to be used when the traffic volume exceeds 1,500 vehicles per hour (Kang et al., 2006). When the system is activated, the value of $D_{\text {LM }}$ corresponds to the increase of $\mathrm{V}_{\mathrm{A}}$. Therefore, the relation between $\mathrm{V}_{\mathrm{A}}, \mathrm{V}_{\mathrm{T}}$ and DLM has to be examined.

The throughput can be influenced by changing the late merge distance, which suggests testing different $\mathrm{D}_{\mathrm{LM}}$. Therefore, simulation is needed for different late merge distance to determine the optimal DLm value. Although the system is designed as DLm equals 1000 
feet, the values of 200, 500 and 1000 feet will be tested.

\subsubsection{Detectors Distance $\left(D_{D}\right)$}

The detectors are connected to the control unit to measure average queue length, speed and traffic volume of the segment in order to trigger late merge system. In most of dynamic late merge systems, multiple sensors are used to recognize high congestion levels. Minnesota DOT uses Remote Traffic Microwave Sensor (RTMS) that is located 1,500 feet from the taper and a Doppler radar installed at the taper. The purpose of RTMS is to measures the average speed of vehicles traveling in both lanes and the total volume, while the Doppler radar measures speed only. Minnesota DOT utilizes RTMS in its late merge system, which is located one mile before the work zone taper. In this system, RTMS-G4 is appointed to provide volume, occupancy, speed and classification information. A single RTMS-G4 can replace multiple inductive loop detectors and supply needed data. The location of RTMS-G4, DD, is 1500 feet from the work zone taper.

\subsubsection{Broadcasting Units $\left(D_{B U 1}\right.$ and $\left.D_{B U 2}\right)$}

One of the main additional components in the CLMS are broadcasting unites that communicant with agents in CVs and broadcast the upper and lower bound of restricted merge areas. CV's drivers should receive the notification about the restricted areas at the same time they can start reading the sign. The PCMs are assumed to have a standard letter height of 18 inches, which gives a best impact reading distance of 180 feet and a maximum readable distance of 750 feet. Therefore, it would be reasonable to receive the notification within 750 feet from the PCMs (Ullman et al., 2005). 
Since the range of communication of the broadcasting unit can cover 750 feet, it will be placed with the first "USE BOTH LANES" sign (DBU1 = 3140 feet) with a communication range setting of 750 feet. Therefore, the distance of "USE BOTH LANES" message from the taper as the following:

$$
\gamma=\alpha+D_{B U 1}
$$

Where:

$\gamma$ is USE BOTH LANES sign distance;

$\alpha$ is a constant of 750 feet; and

DвU1 is distance of the first broadcasting unit

The second Broadcasting Unit is placed with the "MERGE HERE" sign (DBu2 $=1000$ feet, 500 feet, or 200 feet) according to the Late Merge Area. However, as this type of message instruct drivers to merge at a certain point, it would be reasonable to notify CVs about merging area as close to the sign as possible. Therefore, the best impact reading distance will be used as a communication range setting of 180 feet. The distance of "MERGE HERE" message from the taper as the following:

$$
\gamma=\alpha+D_{B U 1}
$$

Where:

$\gamma$ is Merge Here Message sign distance;

$\alpha$ is a constant of 180 feet; and 
$D_{B U 1}$ is distance of the first broadcasting unit

All mentioned distances are taken into consideration in the experiment design and it is planned to investigate the impact of these distances on the operations of the CLMS-Level 1. The following table summarizes the values of all distances considered in this system:

Table 4 Values for Distances in CLMS

\begin{tabular}{|l|l|}
\hline Distance & Value (ft) \\
\hline$D_{N M}$ & 3140 \\
\hline$D_{L M}$ & Variable (e.g. 200, 500, 1000) \\
\hline$D_{D}$ & 1500 \\
\hline$D_{B U 1}$ & 3140 \\
\hline$D_{B U 2}$ & Variable (e.g. 200, 500, 1000) \\
\hline
\end{tabular}

\subsubsection{Compliance Rate Effect}

Compliance rate plays a significant role in the model and influences the effectiveness of late merge system. A study from Pennsylvania DOT revealed that late merge signs are confusing and drivers believe that other drivers typically do not follow the instructions of signs. Hence, there is a lack of effective communication between the traffic control devices and the drivers. As CV displays instructions for the driver on dashboard, the lack of communication can be improved and more CVs would merge in the right position. Therefore, the compliance rate of $\mathrm{CV}$ and non-equipped vehicles can be different. However, measuring the difference in terms of compliance rate between CV and nonequipped vehicles has been unexamined and it would require field study. In this experiment, $\mathrm{CV}$ and non-equipped vehicles will be tested under various compliance rate $(\sigma)$. In non-equipped vehicles, Michigan DOT study found that the average compliance 
rate of merging closer to the taper for drivers can reach $60 \%$, while Minnesota DOT observed $30 \%$ to $40 \%$ of compliance rate. Another compliance rate, which can influence the effectiveness of the system, is the compliance rate of cooperative merge. Again, the display of instructions about merging using DVI in CVs would improve late merge and cooperative merge probability comparing with non-equipped vehicles. Also, DVI would encourage CVs in the closed lane to make merging decision and accept relatively smaller gaps comparing to non-equipped vehicle. Thus, the main difference between CVs and non-equipped vehicles in terms of gap acceptance will be reflected in the lane changing model (see next section). As mentioned earlier, measuring the difference between CV and non-equipped vehicles in terms of compliance rate is unexamined. However, the instructions displayed by DVI is assumed to increase the compliance rate by certain percentage comparing to non-equipped vehicles. The relation between CVs and nonequipped vehicles is described as the following:

$$
\begin{aligned}
& \sigma_{N V}=\sigma \\
& \sigma_{C V}=\sigma_{N V}+x
\end{aligned}
$$

Where:

$\sigma$ is the general compliance rate;

$\sigma_{N V}$ is the compliance rate by non-equipped vehicles;

$\sigma_{C V}$ is the compliance rate by $\mathrm{CV}$; and

$x$ is a variable. 
Substantially, the system is influenced by compliance rates in four different cases including CVs for No Merge Area, non-equipped vehicles for No Merge Area, CVs for cooperative merge and non-equipped vehicles for cooperative merge.

The following table summarizes the four different cases, where compliance rate can influence the system:

Table 5 Compliance Rates Considered in CLMS

\begin{tabular}{|c|c|c|c|c|}
\hline $\begin{array}{l}\text { Compliance } \\
\text { Rate }\end{array}$ & Parameter & Value & $\begin{array}{l}\text { Impacted } \\
\text { Lane }\end{array}$ & $\begin{array}{l}\text { Communication } \\
\text { Ways }\end{array}$ \\
\hline $\begin{array}{l}\text { Non-equipped } \\
\text { vehicles for No } \\
\text { Merge Area }\end{array}$ & $\sigma \mathrm{NM}, \mathrm{NV}$ & $30 \%$ and $60 \%$ & Closed lane & Windows \\
\hline $\begin{array}{l}\text { CVs for No } \\
\text { Merge Area }\end{array}$ & $\sigma_{\mathrm{NM}} \mathrm{CV}$ & $\begin{array}{l}40 \%, 50 \%, \\
60 \%, 70 \%, 80 \\
\text { and } 90 \%\end{array}$ & Closed lane & $\begin{array}{l}\text { Windows and } \\
\text { In-Vehicle }\end{array}$ \\
\hline $\begin{array}{l}\text { Non-equipped } \\
\text { vehicles for } \\
\text { cooperative } \\
\text { merge }\end{array}$ & $\sigma_{\mathrm{C}, \mathrm{NV}}$ & $30 \%$ and $60 \%$ & Open lane & Windows \\
\hline $\begin{array}{l}\text { CVs for } \\
\text { cooperative } \\
\text { merge }\end{array}$ & $\sigma_{\mathrm{C}, \mathrm{CV}}$ & $\begin{array}{l}40 \%, 50 \%, \\
60 \%, 70 \%, 80 \\
\text { and } 90 \%\end{array}$ & Open lane & $\begin{array}{l}\text { Windows and } \\
\text { In-Vehicle }\end{array}$ \\
\hline
\end{tabular}

\subsubsection{System Operations}

As previously mentioned, late merge system is to be implemented when arriving vehicles rate exceeds the threshold. Maryland State of Highway Administration activates late 
merge when the system detects occupancy rate of $15 \%$. However, some of the systems designed by DOTs use the speed as a threshold to activate late merge. When the average speed measured by the detector is less than the trigger speed, late merge is activated. In $70 \mathrm{mph}$ speed limit site, Michigan DOT uses thresholds of $35 \mathrm{mph}$ or $45 \mathrm{mph}$ to trigger the system for a period of at least five minutes. (Weng and Meng., 2011) found that in a work zone with speed limit of $60 \mathrm{mph}$, congestion observed and queue started to build up at speed of approximately $45 \mathrm{mph}$ and $35 \mathrm{mph}$, and arriving vehicles rate of approximately $900 \mathrm{vphpl}$ and 1,000 vphpl. However, the arriving vehicles rate is measured in vehicle per hour in the open lane only, which does not represent the total arriving vehicles rate.

From all mentioned studies, it appears that late merge is implemented when the average speed dropped to a lower level between $35 \mathrm{mph}$ and $50 \mathrm{mph}$. In this system, several traffic volumes will be tested to find when late merge could improve the throughput. Then, late merge in the CLMS can be activated when the sensors detect a certain traffic volumes and deactivate it in all other cases.

\subsubsection{Traffic Flow Modeling in CLMS Level 1}

Modeling the traffic flow considers mainly the movement in longitudinal axis and lateral axis. Car-following model represents the longitudinal movement, while lane-changing represents the lateral movement in traffic flow modeling. 


\subsubsection{Car-Following}

There are different models that describe the car-following and they can be used according to the considered parameters and the purpose of the study. The traffic microsimulation software, VISSIM, utilizes Wiedmann 99, which is a psycho-physical model constituted by several thresholds. Wiedemann 99 model consists of ten calibration parameters, where each of them controls a particular aspect of the car-following model. Table 7 summarizes the parameters that control the car-following model in VISSIM. 
Table 6 Parameters of VISSIM Car-following Model (PTV, 2016)

\begin{tabular}{|c|c|c|}
\hline Parameter & Description & $\begin{array}{l}\text { Default } \\
\text { Value }\end{array}$ \\
\hline $\mathrm{CC} 0$ & $\begin{array}{l}\text { Standstill distance: } \\
\text { Desired distance between lead and following vehicle at } \mathrm{v} \\
=0 \mathrm{mph}\end{array}$ & $4.92 \mathrm{ft}$ \\
\hline $\mathrm{CC} 1$ & $\begin{array}{l}\text { Headway Time: } \\
\text { Desired time in seconds between lead and following } \\
\text { vehicle }\end{array}$ & $0.90 \mathrm{sec}$ \\
\hline $\mathrm{CC} 2$ & $\begin{array}{l}\text { Following Variation: } \\
\text { Additional distance over safety distance that a vehicle } \\
\text { requires }\end{array}$ & $13.12 \mathrm{ft}$ \\
\hline $\mathrm{CC} 3$ & $\begin{array}{l}\text { Threshold for Entering 'Following' State: Time in } \\
\text { seconds before a vehicle starts to decelerate to reach } \\
\text { safety distance (negative) }\end{array}$ & $-8.00 \mathrm{sec}$ \\
\hline $\mathrm{CC} 4$ & $\begin{array}{l}\text { Negative 'Following' Threshold: } \\
\text { Specifies variation in speed between lead and following } \\
\text { vehicle }\end{array}$ & $0.35 \mathrm{ft} / \mathrm{s}$ \\
\hline CC5 & $\begin{array}{l}\text { Positive 'Following Threshold': } \\
\text { Specifies variation in speed between lead and following } \\
\text { vehicle }\end{array}$ & $0.35 \mathrm{ft} / \mathrm{s}$ \\
\hline CC6 & $\begin{array}{l}\text { Speed Dependency of Oscillation: } \\
\text { Influence of distance on speed oscillation }\end{array}$ & 11.44 \\
\hline $\mathrm{CC} 7$ & $\begin{array}{l}\text { Oscillation Acceleration: } \\
\text { Acceleration during the oscillation process }\end{array}$ & $0.82 \mathrm{ft} / \mathrm{s}^{2}$ \\
\hline $\mathrm{CC} 8$ & $\begin{array}{l}\text { Standstill Acceleration: } \\
\text { Desired acceleration starting from standstill }\end{array}$ & $11.48 \mathrm{ft} / \mathrm{s}^{2}$ \\
\hline CC9 & $\begin{array}{l}\text { Acceleration at } 50 \mathrm{mph}: \\
\text { Desired acceleration at } 50 \mathrm{mph}\end{array}$ & $4.92 \mathrm{ft} / \mathrm{s}^{2}$ \\
\hline
\end{tabular}

As discussed earlier, cooperative merge influences the headway as the driver would forfeit its desired headway and increase the gap by slowing down to allow other vehicles to merge. Therefore, the headway in the car-following model in this system can be influenced by another driving behavior, which is cooperative merge. 


\subsubsection{Lane Changing}

Lane changing is generally described by unnecessary and necessary decisions. First, unnecessary decision can happen in free lane-changing statues, where the level of traffic volume is low. On the other hand, necessary lane-changing happens when the vehicle must change the lane because of an obstacle or emergency case. A necessary lanechanging is defined in VISSIM as lane change that is necessary for a vehicle to reach its final destination in the network (PTV, 2016). VISSIM lane changing behavior is characterized by maximum and accepted deceleration rates for the following vehicle.

As the system is built on the concept of cooperation between drivers in late merge area where one lane is closed and lane-changing is necessary, rule-based lane changing model estimated by Hidas (2005) is adopted to add cooperative merge behavior to VISSIM lanechanging rules. Lie et al. (2017) also used Hidas model to fine-tuning ADAS algorithm parameters in connected vehicle environment. The model considers the desired spacing $\left(D_{i}\right)$ as a linear function of the speed.

$$
D_{i}=\alpha v_{i}+\beta
$$

Where:

$D_{i}$ is the speed of the vehicle;

$$
v_{i}, \alpha \text { and } \beta \text { are constants. }
$$

Hidas (2005) classifies the necessary lane-changing into forced and cooperative lanechanging. Forced lane-changing, is when the driver is forced by a merging vehicle to slow down suddenly, which causes emergency breaking. However, cooperative lane- 
changing is when a driver slow down to enlarge the gap for the other driver to merge. The interaction in cooperative lane-changing is described by Hidas as the following:

- It starts when the subject vehicle indicates its willing to merge into the target lane,

- Then, the follower vehicle perceives the situation and decides to cooperate by slowing down to create a larger gap,

- Finally, the subject vehicle notices that the follower vehicle cooperated and as a result the gap enough for to executes the maneuver.

In this model, forced lane-changing is neglected, while cooperative merge is integrated into VISSIM lane-changing rules to represent the lateral movement in the system. During free lane-changing status before work zone instructions, Hidas model will be fully utilized.

The lane-changing model by Hidas (2005) is influenced by several factors as summarized in the following table:

Table 7 Parameters in Hidas Lane_Changing Model

\begin{tabular}{|l|l|}
\hline Parameter & Description \\
\hline$g_{l}$ & Space Gap between Subject Vehicle and Leading Vehicle \\
\hline $\mathrm{g}_{\mathrm{f}}$ & Space Gap between Subject Vehicle and Following Vehicle \\
\hline $\mathrm{g}_{\mathrm{min}}$ & Minimum Safe Constant Gap \\
\hline $\mathrm{g}_{\mathrm{l}, \mathrm{min}}$ & Minimum Gap with Leading Vehicle for Merging \\
\hline $\mathrm{g}_{\mathrm{f}, \mathrm{min}}$ & Minimum Gap with Following Vehicle for Merging \\
\hline $\mathrm{v}_{\mathrm{s}}$ & Speed of Subject Vehicle \\
\hline $\mathrm{v}_{\mathrm{l}}$ & Speed of Leading Vehicle \\
\hline $\mathrm{v}_{\mathrm{f}}$ & Speed of Following Vehicle \\
\hline $\mathrm{c}_{\mathrm{l}}$ & Acceptable Gap Parameter with Leading Vehicle \\
\hline $\mathrm{c}_{\mathrm{f}}$ & Acceptable Gap Parameter with Following Vehicle \\
\hline
\end{tabular}


The gap between the subject vehicle and leading and following vehicle has to meet a certain criterion as the following:

$$
g_{l} \geq g_{l, \min } \quad \text { and } \quad g_{f} \geq g_{f, \min }
$$

Where:

$g_{l}$ is the space gap between subject vehicle and leading vehicle; and $g_{f}$ is the space gap between subject vehicle and following vehicle.

In cooperative and non-cooperative cases:

$$
\begin{aligned}
& g_{l, \text { min }}=g_{\text {min }}+ \begin{cases}c_{l}\left(v_{s}-v_{l}\right) & \text { if } v_{s}>v_{l} \\
0 & \text { otherwise }\end{cases} \\
& g_{f, \text { min }}=g_{\text {min }}+\left\{\begin{array}{cl}
c_{f}\left(v_{f}-v_{s}\right) & \text { if } v_{f}>v_{s} \\
0 & \text { otherwise }
\end{array}\right.
\end{aligned}
$$

Where:

$g_{\text {min }}$ is a minimum safe constant gap (jam gap);

$c_{l}$ and $c_{f}$ are constants;

$v_{s}$ is the speed of the subject vehicle;

$v_{l}$ is the speed of the leading vehicle; and

$v_{f}$ is the speed of following vehicle

As mentioned earlier, the instructions from DVI would encourage CVs in the closed lane to make merging decision and accept relatively smaller gap comparing with nonequipped vehicle. Therefore, Acceptable Gap Parameter with Leading and Following 
Vehicles, $c_{l}$ and $c_{f}$ respectively, can be changed in $\mathrm{CVs}$ to reflect the difference with non-equipped vehicles. However, there is a lack of studies that investigate the effect of $\mathrm{CV}$ on the gap acceptance. Hence, a new parameter will be introduced in this model to reflect the percentage of reduction in the gap acceptance for CVs. In Hidas (2005), $c_{l}$ and $c_{f}$ were estimated from the collected video data and modified for an average of 0.9 . The same parameter value will be used for non-equipped vehicles in this study. In contrast, CVs will be assigned with reduced values to reflect the expected reduction of gap acceptance parameter. Two values are chosen to represent the percentage of reduction (20\% and $40 \%$ ). The following table summarizes acceptable gap parameter values for CV and non-CV:

Table 8 Acceptable Gap Parameters

\begin{tabular}{|c|l|l|}
\hline Parameter & Non-equipped Vehicle & CV \\
\hline$c_{l}$ & 0.9 & $0.9-20 \%=0.72$ \\
& & $0.9-40 \%=0.54$ \\
\hline$c_{f}$ & 0.9 & $0.9-20 \%=0.72$ \\
& & $0.9-40 \%=0.54$ \\
\hline
\end{tabular}

The minimum safe constant gap $\left(g_{\min }\right)$ is a parameter that varies from a driver to another. Hidas model considers a minimum safe constant gap of (6.7 feet) and does not specify the difference with heavy vehicles. Heavy vehicles require about double the distance that passenger vehicles require (Nobukawa et al., 2016). Therefore, in the case of heavy vehicles, $g_{\min }$ is considered as (13.4 feet)

Table 9 Minimum safe constant gaps

\begin{tabular}{|c|l|l|}
\hline Parameter & Passenger Vehicle & Heavy Vehicle \\
\hline$g_{\min }$ & 6.7 feet & 13.4 feet \\
\hline
\end{tabular}


In CLMS, several lane-changing models will be used along the freeway and they are different according to the area. In early merge area where there are no instructions, Hidas 2005 is used with free lane-changing. In no merge area, Hidas 2005 is utilized with (No Merge Effect) as drivers are instructed to stay on lane. Eventually, late merge takes place using CLMS based on Hidas 2005 Model (See Figurer 20)

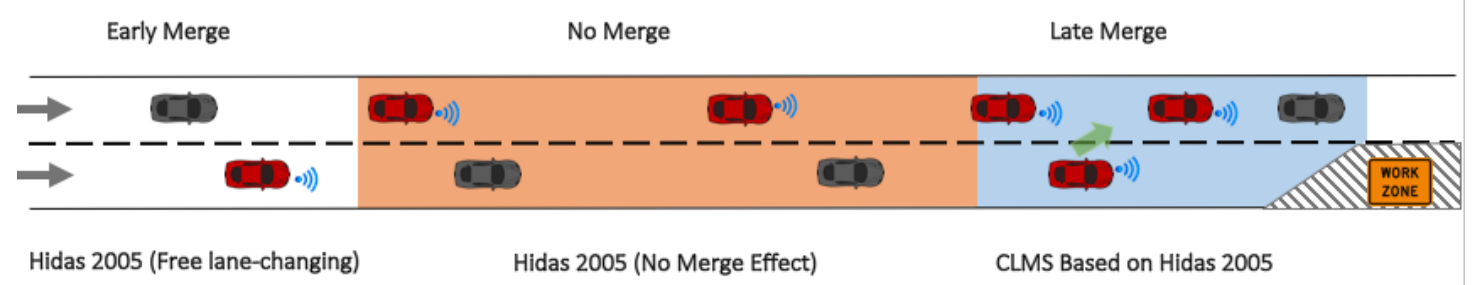

Figure 20 Summary of Lane-changing Models in CLMS

\subsubsection{Simulation Modeling}

Microscopic traffic simulation is an effective method in traffic operations to evaluate multiple scenarios as it considers the effects of microscopic characteristics such as driver behavior and vehicle characteristics (Elefteriadou, 2014). VISSIM is a well-known microscopic modeling software that can be used to model various scenarios of traffic operations (PTV Vision, 2016).

A two-lane freeway section on Interstate 64 between Louisville and Frankfort, Kentucky was modeled in VISSIM using links and connectors. The drop of one lane was modeled using connectors to connect the two lanes to a one lane work zone to represent the lane 
closure configuration. A static route decision started at the beginning of the network till the end to ensure that the number of vehicles entering the freeway is equal to the number of vehicles going through the work zone and leaving the network. The lane-changing model was modified to reflect Hidas 2005 with cooperation effect by developing a new $\mathrm{C}++$ code. The compiled code in Dynamic Link Library (DLL) extension was added as an external driving model to control lane-changing process each 0.1 second.

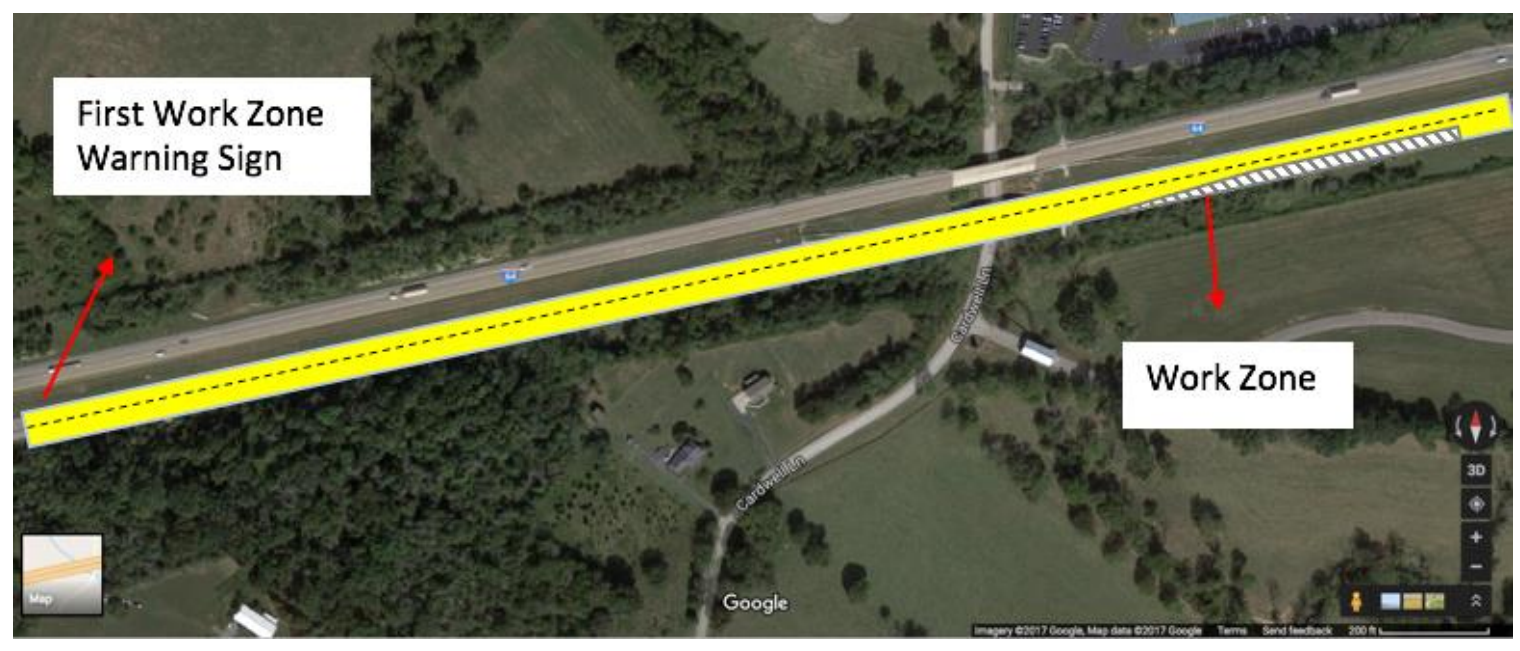

Figure 21 The Actual Site of Simulated Experiment (I-64 East)

\subsubsection{Experimental Design}

In this work, the simulation is developed to study the capacity of work zones and the effect of several variables on the system. However, due to the difference of the layout and components of the three systems, each one includes different number of examined variables. 


\section{System 1: Traditional Work Zone}

In traditional work zone, drivers are warned about the work zone, but there is no control strategy for the merging process. Therefore, the variables of traditional work zone in this experiment are limited to the traffic characteristics such as traffic volume, heavy vehicles percentage, and lane distribution. Traffic volumes include low, moderate and high numbers for two-lane freeway (1200, 1600, 2000, 2400, 2800 and $3200 \mathrm{veh} / \mathrm{h})$. Heavy vehicles have different driving behavior comparing to passenger vehicles due to the difference in size and weight. Thus, the effect of heavy vehicles on the system will be tested by three levels $(0 \%, 10 \%$ and $20 \%)$. As the scenario is built on a two-lane freeway that drops to one lane, the effect of the traffic distribution onto the two lanes will be considered $(30 \%|70 \%, 50 \%| 50 \%$ and $70 \% \mid 30 \%)$ where the first percentage is for the right closed lane and the second percentage is for left open lane.

\section{System 2: Work Zone with Late Merge Strategy}

Beside the three traffic characteristics in traditional work zone, late merge control strategy includes additional variables such as late merge area and compliance rate. As mentioned earlier, the area where drivers are instructed to merge-in varies from system to another. Therefore, it is vital in this study to consider three levels of late merge area (200 feet, 500 feet and 1000 feet). Previously mentioned studies (Table 2) about late merge have found that compliance rate varies from $30 \%$ to $65 \%$. In this study, the impact of compliance rate of non-CV will be studied though two levels (30\% and 60\%). 


\section{System 3: CLMS (Level 1)}

In addition to all mentioned variables in System 1 and System 2, the CLMS will consider compliance rate for $\mathrm{CV}$, gap acceptance reduction and market penetration rate. $\mathrm{CV}$ are expected to have higher compliance rate due to the fact that drivers are notified by both signs and DVI. Therefore, the influence of several compliance rate levels will be studied $(50 \%, 60 \%, 70 \%, 80 \%, 90 \%$ and $100 \%)$. Also, the influence of the expected gap acceptance reduction will be tested through two levels $(20 \%$ and $40 \%)$. The percentage of $\mathrm{CV}$ out of the total passenger cars in the system will be considered by 5 levels including $(20 \%, 40 \%, 60 \%, 80 \%$ and $100 \%)$.

The following table summarizes the examined variables:

Table 10 Variables Considered in Experiment Design

\begin{tabular}{|c|c|c|c|c|c|}
\hline Variable & $\begin{array}{l}\text { Number of } \\
\text { Levels }\end{array}$ & Value & $\begin{array}{l}\text { Traditional } \\
\mathrm{WZ}\end{array}$ & $\begin{array}{l}\text { Late } \\
\text { Merge } \\
\text { WZ }\end{array}$ & CLMS \\
\hline \multirow{6}{*}{$\begin{array}{l}\text { Traffic Volume } \\
(\text { veh/h) }\end{array}$} & \multirow{6}{*}{6} & 1200 & \multirow{6}{*}{$\checkmark$} & \multirow{6}{*}{$\checkmark$} & \multirow{6}{*}{$\checkmark$} \\
\hline & & 1600 & & & \\
\hline & & 2000 & & & \\
\hline & & 2400 & & & \\
\hline & & 2800 & & & \\
\hline & & 3200 & & & \\
\hline \multirow{3}{*}{$\begin{array}{l}\text { Heavy Vehicles } \\
\text { Percentage (\%) }\end{array}$} & \multirow{3}{*}{3} & $0 \%$ & \multirow{3}{*}{$\checkmark$} & \multirow{3}{*}{$\checkmark$} & \multirow{3}{*}{$\checkmark$} \\
\hline & & $10 \%$ & & & \\
\hline & & $20 \%$ & & & \\
\hline \multirow{3}{*}{$\begin{array}{l}\text { Lane Distribution } \\
(\% / \text { lane })\end{array}$} & \multirow{3}{*}{3} & $30 \%$ & \multirow{3}{*}{$\checkmark$} & \multirow{3}{*}{$\checkmark$} & \multirow{3}{*}{$\checkmark$} \\
\hline & & $50 \%$ & & & \\
\hline & & $70 \%$ & & & \\
\hline \multirow{4}{*}{$\begin{array}{l}\text { Late Merge } \\
\text { Distance (feet) }\end{array}$} & \multirow{3}{*}{3} & 200 & & \multirow{3}{*}{$\checkmark$} & \multirow{3}{*}{$\checkmark$} \\
\hline & & 500 & & & \\
\hline & & 1000 & & & \\
\hline & 2 & $20 \%$ & & & $\checkmark$ \\
\hline
\end{tabular}




\begin{tabular}{|c|c|c|c|c|}
\hline $\begin{array}{l}\text { Reduction in Gap } \\
\text { Acceptance }(\%)\end{array}$ & & $40 \%$ & & \\
\hline \multirow{5}{*}{$\begin{array}{l}\text { Market Penetration } \\
\text { Rate }(\%)\end{array}$} & \multirow{5}{*}{5} & $20 \%$ & & \multirow{5}{*}{$\checkmark$} \\
\hline & & $40 \%$ & & \\
\hline & & $60 \%$ & & \\
\hline & & $80 \%$ & & \\
\hline & & $100 \%$ & & \\
\hline \multirow{2}{*}{$\begin{array}{l}\text { Non-CV } \\
\text { Compliance Rate } \\
(\%)\end{array}$} & \multirow[b]{2}{*}{2} & $30 \%$ & \multirow[b]{2}{*}{$\checkmark$} & \multirow[b]{2}{*}{$\checkmark$} \\
\hline & & $60 \%$ & & \\
\hline \multirow{6}{*}{$\begin{array}{l}\text { CV Compliance } \\
\text { Rate }\end{array}$} & \multirow{6}{*}{6} & $50 \%$ & & \multirow{6}{*}{$\checkmark$} \\
\hline & & $60 \%$ & & \\
\hline & & $70 \%$ & & \\
\hline & & $80 \%$ & & \\
\hline & & $90 \%$ & & \\
\hline & & $100 \%$ & & \\
\hline
\end{tabular}

Taking into consideration that there should be a scenario for each combination of all mentioned variables, the numbers of simulation scenario as the following:

Table 11 Summary of Number of Simulation Scenarios

\begin{tabular}{|l|l|}
\hline System & Simulation Scenarios \\
\hline Traditional Work Zone & 54 \\
\hline Late Merge Work Zone & 324 \\
\hline CLMS & 1692 \\
\hline Total Simulation Scenarios & 2070 \\
\hline
\end{tabular}

It is worth mentioning that testing all combinations of variables in CLMS will result an excessive number of scenarios (19440) that would need a year of continuous running. Hence, all levels of variables will be considered, while the compliance rate of CV will be fixed. Then, the combinations of the compliance rate levels of $\mathrm{CV}$, non-CV and traffic 
volumes will be tested, while other variables are fixed. This way, a reasonable number of simulation scenarios can be conducted.

\section{Required Number of Runs for Each Simulation Scenario}

The sample size equation modified by Virginia Department of Transportation is a tool to determine the required number of simulation runs.

$$
N=\frac{(Z)^{2}\left(S_{S}\right)^{2}}{E^{2}}
$$

Where:
$N$ is necessary sample size;
$Z$ is the number of standard deviations away from the mean corresponding to the desired confidence level;
$S$ is sample standard deviation; and
$E$ is tolerable error in terms of the sample mean.

This methodology is considered as an iterative process and each new round of calculations have to be performed on the results of the necessary sample runs to see if the numbers are adequate. Taking into consideration that this process, in the case of several dependent and independent variable, may become time consuming and costly, a threshold of 30 runs can be established (Virginia Department of Transportation, 2013). Thus, this experiment will consider using examine each scenario with 30 runs. Given that the number of simulation scenarios is 2070 , the total number of runs is 62,100 . 


\subsubsection{Measure of Effectiveness}

The effectiveness of a system can be seen through the improvement of certain measurements. By consulting previous literature, the major criterion of work zone improvement is measured by the increase of throughput. Also, other criteria such as the overall delay and the queue length can be analyzed to evaluate the improvement of the level of service (Elefteriadou, 2014). The following measure of effectiveness (MOEs) will be considered:

- Throughput: the number of vehicle passing through the one-lane work zone per hour (veh/h).

- Capacity: the maximum traffic demand the system can efficiently operate

- V/C: volume-to-capacity ratio where 1 is when volume approach capacity and highway segment is fully saturated. $90 \%$ degree of saturation is the targeted threshold (Systematics, 2009)

- Overall Delay: the additional time taken by the vehicle to travel the whole corridor comparing with the ideal time to do that with the posted speed limit (sec/veh)

Overall Delay $=$ actual travel time - ideal time

- Queue Length: the length of the formed queue from the taper (feet) 
Table 12 Measure of Effectiveness (MOEs)

\begin{tabular}{|l|l|l|}
\hline MOEs & Unit & Description \\
\hline Throughput & Veh/h & $\begin{array}{l}\text { It reflects the work zone output in terms of } \\
\text { vehicles per hour. }\end{array}$ \\
\hline Capacity & Veh/h & $\begin{array}{l}\text { It represents the work zone intake in terms } \\
\text { on vehicles per hour. }\end{array}$ \\
\hline V/C & Ratio & $\begin{array}{l}\text { It reflects the degree of saturation under } \\
\text { different traffic demands. }\end{array}$ \\
\hline Delay & Sec/veh & $\begin{array}{l}\text { It describes the cost of the work zone in } \\
\text { terms of seconds per vehicle. }\end{array}$ \\
\hline Queue Length & feet & $\begin{array}{l}\text { It indicates the length of the traffic backup } \\
\text { and can be very important in case of the } \\
\text { existence of nearby ramps. }\end{array}$ \\
\hline
\end{tabular}

To measure the mentioned performance measures, couple of statistical tests need to be implemented. Using Minitab Statistical Software, multiple regression analysis and T-tests are completed with $95 \%$ confidence interval. 


\section{CHAPTER IV}

\section{ANALYSIS OF OPERATIONS PREFORMANCE}

The analysis of the CLMS is performed with consideration of traditional work zone system and late merge system. It starts with the performance of CLMS and other system by determining significant variables that impact operations. The relation between significant variables and the MOEs is illustrated by multiple linear regression models. Multiple linear regression models take the following form:

$$
Y_{i}=\beta_{0}+\beta_{1} X_{i}+\varepsilon_{i}
$$

Where:

$Y_{i}$ is the dependent variable;

$X_{i}$ is the level of the independent (predictor) variable;

$\beta_{0}$ is the mean of $\mathrm{Y}$ when $\mathrm{X}=0$ ( $\mathrm{Y}$-intercept);

$\beta_{1}$ is the change in mean of $\mathrm{Y}$ when $\mathrm{X}$ increases by 1 (slope); and

$\varepsilon_{i}$ is the random error term (constant).

MOEs are plotted with significant variables, assuming some general traffic characteristics such as heavy vehicle percentage. To demonstrate the difference between the three systems, t-tests were conducted using a software package (Minitab). 


\subsection{CLMS Performance}

As previously mentioned, MOEs are dependent variables while the rest of variable are independent. The following table summarizes the dependent variables with their symbols, values range and applicable system.

Table 13 Description of Variables in Compared Systems

\begin{tabular}{|c|c|c|c|}
\hline Variable & Symbol & Values Range & System \\
\hline Traffic Demand & $\gamma$ & $1200 \mathrm{veh} / \mathrm{h}-3200 \mathrm{veh} / \mathrm{h}$ & $\begin{array}{l}\text { Traditional WZ } \\
\text { Late Merge System } \\
\text { CLMS }\end{array}$ \\
\hline Lane Distribution & $\rho$ & $\begin{array}{l}30|70 \%, 50 \%| 50 \%, \\
70 \% \mid 30 \% \\
\text { LD = (right lane } \\
\text { percentage) }\end{array}$ & $\begin{array}{l}\text { Traditional WZ } \\
\text { Late Merge System } \\
\text { CLMS }\end{array}$ \\
\hline $\begin{array}{l}\text { Heavy Vehicle } \\
\text { Percentage }\end{array}$ & $\varphi$ & $0 \%-20 \%$ & $\begin{array}{l}\text { Traditional WZ } \\
\text { Late Merge System } \\
\text { CLMS }\end{array}$ \\
\hline Late Merge Area & $\psi$ & 200 feet -1000 feet & $\begin{array}{l}\text { Late Merge System } \\
\text { CLMS }\end{array}$ \\
\hline $\begin{array}{l}\text { Compliance Rate for } \\
\mathrm{CV}\end{array}$ & $\sigma_{C V}$ & $50 \%-100 \%$ & CLMS \\
\hline $\begin{array}{l}\text { Compliance Rate for } \\
\text { Non-CV }\end{array}$ & $\sigma_{\text {Non-CV }}$ & $30 \%$ and $60 \%$ & $\begin{array}{l}\text { Late Merge System } \\
\text { CLMS }\end{array}$ \\
\hline $\begin{array}{l}\text { Reduction Factor for } \\
\text { Gap Acceptance }\end{array}$ & $F$ & $20 \%$ and $40 \%$ & CLMS \\
\hline $\begin{array}{l}\text { Market Penetration } \\
\text { Rate }\end{array}$ & $\omega$ & $20 \%-100 \%$ & CLMS \\
\hline
\end{tabular}

There are certain variables that all systems have such as traffic demand, heavy vehicles percentage, and lane distribution. Some other variables are uniquely related to a specific system such as reduction factor and market penetration rate. The following table summarizes the potential variables that may affect each system: 
Table 14 Potential Contributing Factors for All Systems

\begin{tabular}{|l|l|l|l|l|c|c|c|c|}
\hline System & \multicolumn{9}{|c|}{ Variable } \\
\hline Traditional Work Zone & $\gamma$ & $\rho$ & $\varphi$ & - & - & - & - & - \\
\hline Late Merge & $\gamma$ & $\rho$ & $\varphi$ & $\psi$ & $\sigma_{\text {Non-CV }}$ & - & - & - \\
\hline CLMS & $\gamma$ & $\rho$ & $\varphi$ & $\psi$ & $\sigma_{\text {Non-CV }}$ & $\sigma_{C V}$ & $F$ & $\omega$ \\
\hline
\end{tabular}

\subsubsection{Work Zone Throughput}

Throughput is the measurement of number of vehicles that can proceed through a certain point in an hour. The higher number of throughput indicates the ability of the system to move vehicles efficiently. Capacity of work zone is also represented by the maximum throughput of the system. Therefore, throughput has to be investigated to find the capacity of the work zone. Each system has different variables that affect the throughput and hence impact the capacity of work zone.

In traditional work zone, traffic demand is the only significant variable impacting throughput of the system. The two other considered variables, heavy vehicle percentage and lane distribution, have no significant effect on the system. With the traffic backup in the open lane, lane distribution and heavy vehicle were not significantly able to change throughput. The following table summarizes the statistical test of throughput in traditional work zone system:

Table 15 Statistical Test for Throughput in Traditional Work Zone

\begin{tabular}{|l|l|l|l|l|l|}
\hline Variable & Coef & SE Coef & T-Value & P-Value & VIF \\
\hline Constant & 1105.9 & 51.6 & 21.43 & 0.000 & \\
\hline$\varphi$ & 251 & 134 & 1.87 & 0.067 & 1.00 \\
\hline$\gamma$ & 0.1421 & 0.0160 & 8.87 & $0.000^{*}$ & 1.00 \\
\hline$\rho$ & 34.7 & 67.0 & 0.52 & 0.607 & 1.00 \\
\hline
\end{tabular}

* Significant Variable 
By considering the significant variable in regression analysis, the following regression model, with $\mathrm{R}^{2}=0.59$, describes throughput of traditional work zone:

$$
\Lambda=1148.4+0.1421 \gamma
$$

Where:
$\Lambda$ is throughput of traditional work zone; and
$\gamma$ is traffic demand

In late merge system, heavy vehicle percentage, traffic demand and compliance rate of non-CV appear as significant variables that affect the throughput of the system. The high number of heavy vehicles would prevent late merge from achieving higher throughput, while the compliance rate would improve throughput by applying late merges.

The following table summarizes the statistical test of throughput in late merge system:

Table 16 Statistical Test for Throughput in Late Merge System

\begin{tabular}{|l|l|l|l|l|l|}
\hline Variable & Coef & SE Coef & T-Value & P-Value & VIF \\
\hline Constant & 971.1 & 35.3 & 27.53 & 0.000 & \\
\hline$\varphi$ & -129.1 & 40.2 & -3.21 & $0.001^{*}$ & 1.00 \\
\hline$\gamma$ & 0.24602 & 0.00883 & 27.86 & $0.000^{*}$ & 1.00 \\
\hline$\rho$ & 68.5 & 36.9 & 1.85 & 0.065 & 1.00 \\
\hline$\psi$ & -0.0067 & 0.0183 & -0.37 & 0.715 & 1.00 \\
\hline$\sigma_{\text {Non-CV }}$ & -129.1 & 40.2 & -3.21 & $0.001^{*}$ & 1.00 \\
\hline
\end{tabular}

* Significant Variable

By considering the significant variables in regression analysis, the following regression model, with $\mathrm{R}^{2}=0.73$, describes throughput of late merge system: 


$$
\Lambda=1001.6+601.1 \varphi+0.24602 \gamma-129.1 \sigma_{N o n-C V}
$$

Where:
$\Lambda$ is throughput of late merge system;
$\varphi$ is heavy vehicle percentage
$\gamma$ is traffic demand
$\sigma_{\text {Non-CV }}$ is compliance rate for non-CV

In CLMS, heavy vehicle percentage is a significant variable along with the reduction factor of gap acceptance. In fact, accepting a smaller gap for merging increased the throughput of the system. The following table summarizes the statistical test of throughput in the CLMS:

Table 17 Statistical Test for Throughput in CLMS

\begin{tabular}{|l|l|l|l|l|l|}
\hline Variable & Coef & SE Coef & T-Value & P-Value & VIF \\
\hline Constant & 1055.9 & 17.6 & 59.87 & 0.000 & \\
\hline$\varphi$ & 366.9 & 34.7 & 10.57 & $0.000^{*}$ & 1.00 \\
\hline$\gamma$ & 0.24743 & 0.00415 & 59.62 & $0.000^{*}$ & 1.00 \\
\hline$\rho$ & 51.9 & 17.4 & 2.99 & $0.003^{*}$ & 1.00 \\
\hline$\psi$ & -0.00894 & 0.00859 & -1.04 & 0.293 & 1.00 \\
\hline$\omega$ & 9.6 & 10.0 & 0.96 & 0.339 & 1.00 \\
\hline$F$ & -230.4 & 28.4 & -8.13 & $0.000^{*}$ & 1.00 \\
\hline
\end{tabular}

* Significant Variable

By considering the significant variables in regression analysis, the following regression model, with $\mathrm{R}^{2}=0.69$ describes throughput of late merge system:

$$
\Lambda=1056.5+366.9 \varphi+0.24743 \gamma+51.9 \rho-230.4 F
$$


Where:
$\Lambda$ is throughput of CLMS
$\varphi$ is heavy vehicle percentage
$\gamma$ is traffic demand
$\rho$ is lane distribution
$F$ is reduction factor for gap acceptance

The following table summarizes the significant variables affecting vehicle throughput in each system.

Table 18 Summary of Significant Variables Impacting Throughput

\begin{tabular}{|l|l|l|l|l|l|l|c|c|}
\hline System & \multicolumn{9}{|c|}{ Variable } \\
\hline Traditional Work Zone & $\gamma$ & $\rho^{*}$ & $\varphi$ & - & - & - & - & - \\
\hline Late Merge & $\gamma^{*}$ & $\rho^{*}$ & $\varphi$ & $\psi$ & $\sigma_{\text {Non-CV }}$ & - & - & - \\
\hline CLMS & $\gamma^{*}$ & $\rho^{*}$ & $\varphi$ & $\psi$ & $\sigma_{\text {Non-CV }}$ & $\sigma_{C V}$ & $F^{*}$ & $\omega$ \\
\hline
\end{tabular}

* Significant Variable

To compare the three system, only significant variables are taken into consideration.

However, heavy vehicle percentage is assumed as a reasonable value of $10 \%$ for the sake of conciseness. The following graph illustrates the performance of the three systems with significant variables. 


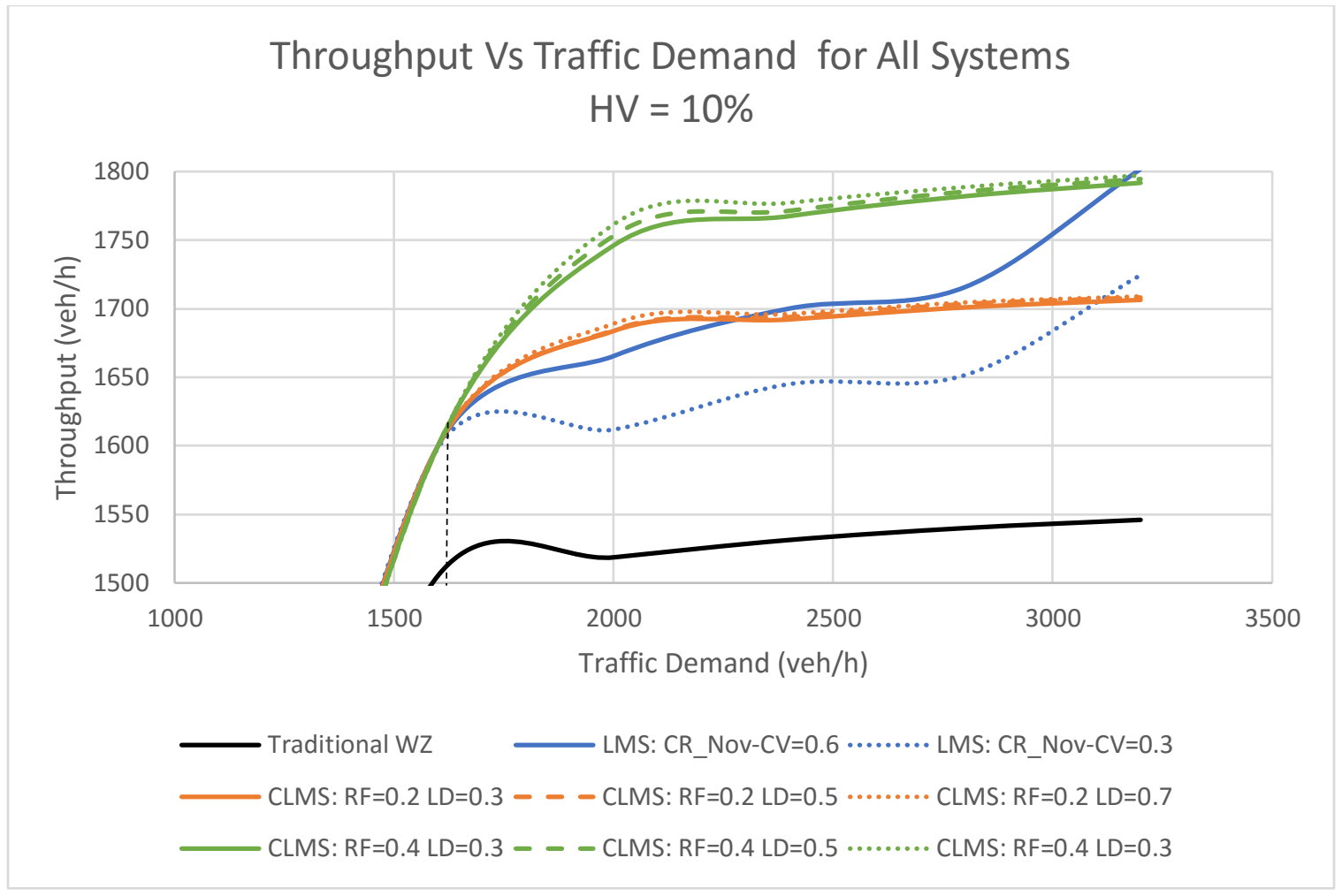

Figure 22 Comparison of Throughput in All Systems

From the figure above, it appears that late merge system is substantially impacted by different compliance rate when the traffic demand increases. On the other hand, the difference between lane distributions in the CLMS is not visually noticed. Comparing performance of late merge system with the CLMS, it appears that at the traffic demand of $1600 \mathrm{veh} / \mathrm{h}$ the CLMS started to perform better. At the traffic demand of $2400 \mathrm{veh} / \mathrm{h}$, the late merge system in the case of $60 \%$ compliance rate started to show an improved performance.

In case of 0.4 reduction factor, the CLMS performed better than late merge system from the traffic demand of $1600 \mathrm{veh} / \mathrm{h}$ till $2800 \mathrm{veh} / \mathrm{h}$ when late merge with the higher compliance rate started to substantially increase. The CLMS, in general, have an 
increased throughout while traffic demands are 2000, 2400 and $2800 \mathrm{veh} / \mathrm{h}$. This because late merge strategy is feasible during low to moderate traffic conditions (Beacher at el., 2004).To test the significance of the difference between the CLMS and late merge system, comparisons between the two systems in case of moderate traffic demands 2000, 2400 and $2800 \mathrm{veh} / \mathrm{h}$ were conducted. The t-test results showed significant difference between late merge system and the CLMS. The following table summarizes t-test results:

Table 19 T-Test Results for the Difference in Throughput for All Systems

\begin{tabular}{|c|c|c|c|c|c|c|c|c|c|c|c|c|}
\hline & \multirow[t]{4}{*}{ TWZ } & \multicolumn{2}{|c|}{ LMS } & \multicolumn{6}{|c|}{ CLMS } \\
\hline & & & & & \multirow{3}{*}{$\begin{array}{l}\begin{array}{l}\sigma \text { Non- } \\
\text { CV }\end{array} \\
0.3\end{array}$} & \multirow{3}{*}{$\begin{array}{l}\begin{array}{l}\text { ONon- } \\
\text { CV }\end{array} \\
0.6\end{array}$} & \multirow{2}{*}{\multicolumn{3}{|c|}{$\begin{array}{c}\mathrm{F}=0.2 \\
\rho\end{array}$}} & \multirow{2}{*}{\multicolumn{3}{|c|}{$F=0.4$}} \\
\hline & & & & & & & & & & & & \\
\hline & & & & & & & 0.3 & 0.5 & 0.7 & 0.3 & 0.5 & 0.7 \\
\hline \multicolumn{13}{|l|}{ TWZ } \\
\hline \multirow[t]{2}{*}{ LMS } & \multicolumn{2}{|c|}{$\begin{array}{l}\sigma_{\text {Non- }} \\
C V\end{array}$} & 0.3 & $0.00^{*}$ & & & & & & & & \\
\hline & \multicolumn{2}{|c|}{$\sigma_{\text {Non-CV }}$} & 0.6 & $0.00^{*}$ & $0.00^{*}$ & & & & & & & \\
\hline \multirow{6}{*}{ CLMS } & \multirow{3}{*}{$\begin{array}{l}\mathrm{F}= \\
0.2\end{array}$} & $\rho$ & 0.3 & $0.00^{*}$ & $0.00 *$ & 0.91 & & & & & & \\
\hline & & $\rho$ & 0.5 & $0.00^{*}$ & $0.00^{*}$ & 0.99 & 0.12 & & & & & \\
\hline & & $\rho$ & 0.7 & $0.00^{*}$ & $0.00 *$ & 0.83 & $0.00^{*}$ & 0.03 & & & & \\
\hline & \multirow{3}{*}{$\begin{array}{l}F= \\
0.4\end{array}$} & $\rho$ & 0.3 & $0.00^{*}$ & $0.00^{*}$ & $0.00^{*}$ & $0.00^{*}$ & $0.00^{*}$ & $0.00^{*}$ & & & \\
\hline & & $\rho$ & 0.5 & $0.00^{*}$ & $0.00 *$ & $0.00^{*}$ & $0.00^{*}$ & $0.00^{*}$ & $0.00^{*}$ & 0.89 & & \\
\hline & & $\rho$ & 0.7 & $0.00^{*}$ & $0.00 *$ & $0.00^{*}$ & $0.00^{*}$ & $0.00^{*}$ & $0.00^{*}$ & 0.73 & 0.83 & \\
\hline
\end{tabular}

*Significant

There is no significant difference

The mean of sample in column is significantly higher than mean of sample in row

The mean of sample in row is significantly higher than mean of sample in column

From T-test results, it appears that there is significant difference between all systems in terms of throughput. The CLMS shows significantly higher throughput than late merge system and traditional work zone as expected. By looking carefully at the comparison table, the CLMS when reduction factor is 0.2 is not significantly different from the late merge system with compliance rate of 0.6 . The effect of reduction factor is noticeable and 
the T-test results confirmed that there is significant difference between 0.2 and 0.4 . The results also indicated lack of difference between lane distribution rates when the reduction factor is 0.4 .

\subsubsection{Work Zone Capacity}

Capacity is measured to determine the maximum number of vehicles that the freeway can efficiently operate. It is represented by the maximum throughput at different traffic demands.

In traditional work zone, lane distribution is the only significant variable impacting capacity of the system. It seems that the capacity is more sensitive to the lane distribution because the highest throughput is influenced by the difference of number of vehicles in each lane. The following table summarizes the statistical test of throughput in traditional work zone system:

Table 20 Statistical Test for Capacity in Traditional Work Zone

\begin{tabular}{|l|l|l|l|l|l|}
\hline Variable & Coef & SE Coef & T-Value & P-Value & VIF \\
\hline Constant & 1537.94 & 1.68 & 913.51 & 0.000 & \\
\hline$\varphi$ & 3.33 & 5.98 & 0.56 & 0.598 & 1.00 \\
\hline$\rho$ & 15.00 & 2.99 & 5.01 & $0.002^{*}$ & 1.00 \\
\hline
\end{tabular}

* Significant Variable

By considering the significant variable in regression analysis, the following regression model, with $\mathrm{R}^{2}=0.80$, describes capacity of traditional work zone:

$$
\Lambda=1538.28+15.00 \rho
$$

Where: 
$\Lambda$ is capacity of traditional work zone

$\rho$ is lane distribution

In late merge system, heavy vehicles percentage and compliance rate of non-CV appear as significant variable that affect the capacity of the system. The high number of heavy vehicles would prevent late merge from achieving higher throughput, while the compliance rate would improve throughput by applying late merges. The following table summarizes the statistical test of throughput in late merge system:

Table 21 Statistical Test for Capacity in Late Merge System

\begin{tabular}{|l|l|l|l|l|l|}
\hline Variable & Coef & SE Coef & T-Value & P-Value & VIF \\
\hline Constant & 1827.6 & 40.0 & 45.67 & 0.000 & \\
\hline$\varphi$ & -195.0 & 96.8 & -2.01 & $0.050^{*}$ & 1.00 \\
\hline$\rho$ & 78.6 & 48.4 & 1.62 & 0.111 & 1.00 \\
\hline$\psi$ & 0.0236 & 0.0239 & 0.99 & 0.328 & 1.00 \\
\hline$\sigma_{\text {Non- } C V}$ & -232.8 & 52.4 & -4.44 & $0.000^{*}$ & 1.00 \\
\hline
\end{tabular}

* Significant Variable

By considering the significant variables in regression analysis, the following regression model, with $\mathrm{R}^{2}=0.32$, describes throughput of late merge system:

$$
\Lambda=1882.9-202.2 \varphi-236.1 \sigma_{\text {Non-CV }}
$$

Where:

$\Lambda$ is capacity of late merge system

$\varphi$ is heavy vehicle percentage

$\sigma_{\text {Non-CV }}$ is compliance rate for non-CV 
In CLMS, heavy vehicle percentage is a significant variable along with the reduction factor of gap acceptance. In fact, accepting smaller gap for merging increased the throughput of the system. The following table summarizes the statistical test of throughput in the CLMS:

Table 22 Statistical Test for Capacity in CLMS

\begin{tabular}{|l|l|l|l|l|l|}
\hline Variable & Coef & SE Coef & T-Value & P-Value & VIF \\
\hline Constant & 1872.3 .3 & 15.4 & 121.79 & 0.000 & \\
\hline$\varphi$ & -181.3 & 35.4 & -5.13 & $0.000^{*}$ & 1.00 \\
\hline$\rho$ & 31.3 & 17.7 & 1.77 & 0.078 & 1.00 \\
\hline$\psi$ & -0.03196 & 0.00875 & -3.65 & $0.000^{*}$ & 1.00 \\
\hline$\omega$ & 3.5 & 10.2 & 0.34 & 0.735 & 1.00 \\
\hline$F$ & -308.3 & 28.9 & -10.67 & $0.000^{*}$ & 1.00 \\
\hline
\end{tabular}

* Significant Variable

By considering the significant variables in regression analysis, the following regression model, with $\mathrm{R}^{2}=0.37$, describes throughput of late merge system:

$$
\Lambda=1890.0-181.3 \varphi-0.03196 \psi-308.3 F
$$

Where:

$$
\begin{aligned}
& \Lambda \text { is capacity of CLMS } \\
& \varphi \text { is heavy vehicle percentage } \\
& F \text { is reduction factor for gap acceptance }
\end{aligned}
$$

The following table summarizes the significant variables affecting vehicle capacity in each system: 
Table 23 Summary of Significant Variables Impacting Capacity

\begin{tabular}{|l|l|l|l|l|l|l|c|c|}
\hline System & \multicolumn{9}{|c|}{ Variable } \\
\hline Traditional Work Zone & $\gamma$ & $\rho^{*}$ & $\varphi^{*}$ & - & - & - & - & - \\
\hline Late Merge & $\gamma$ & $\rho$ & $\varphi^{*}$ & $\psi$ & $\sigma_{\text {Non-CV }}$ & - & - & - \\
\hline CLMS & $\gamma$ & $\rho$ & $\varphi^{*}$ & $\psi$ & $\sigma_{\text {Non-CV }}$ & $\sigma_{C V}$ & $F^{*}$ & $\omega$ \\
\hline
\end{tabular}

* Significant Variable

To compare the three system, only significant variables are taken into consideration.

However, heavy vehicle percentage is assumed as a reasonable value of $10 \%$ for the sake of conciseness. The following graph illustrates the performance of the threes systems with significant variables.

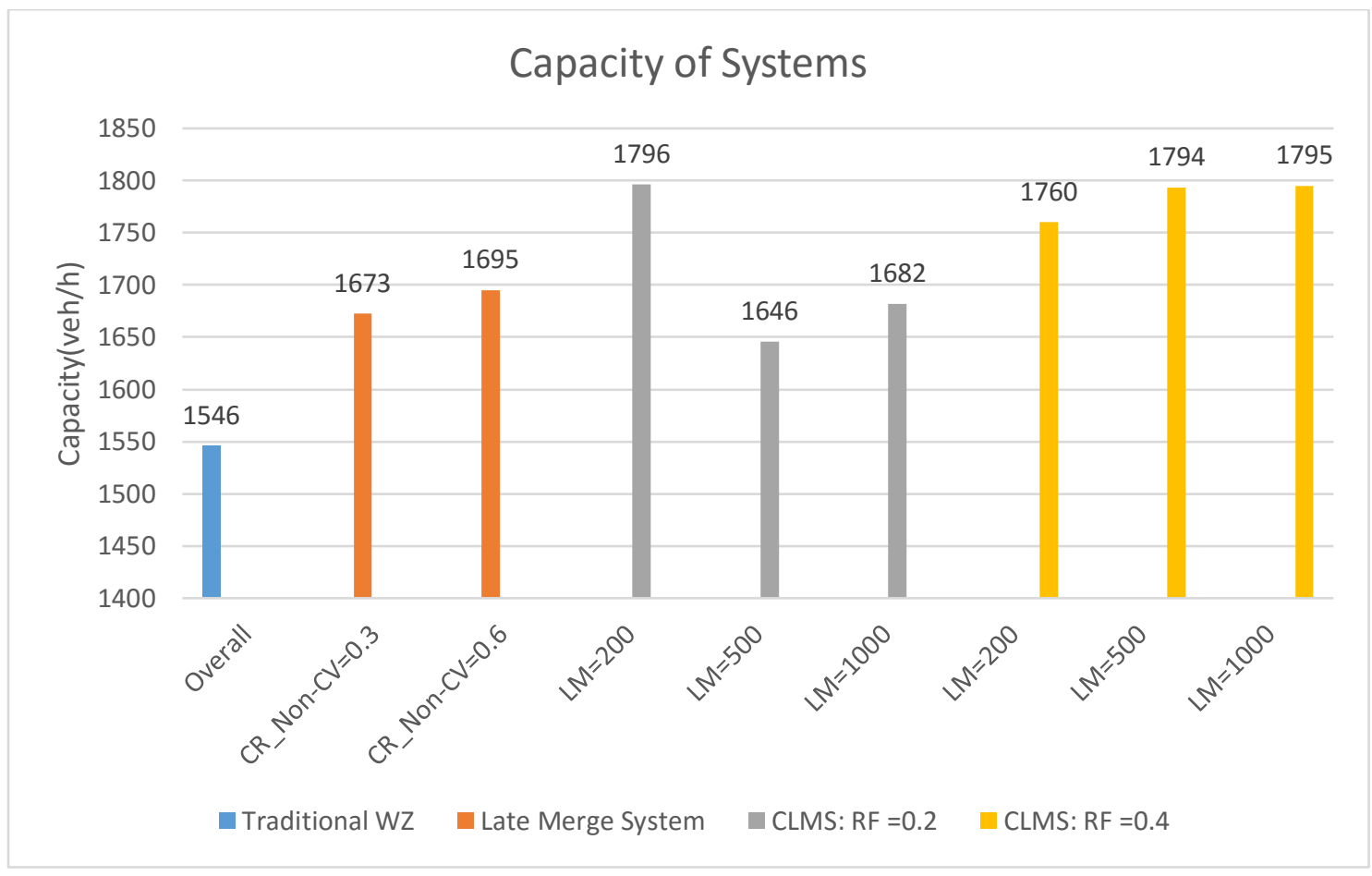

Figure 23 Comparison of Capacity for All Systems 
By looking at Figure 23, the CLMS shows relatively higher capacities comparing to other systems. The effect of compliance rate on late merge is noticed as $60 \%$ compliance rate would improve the capacity of late merge system. The CLMS is influenced by both reduction factor and late merge area.

Table 24 T-Test Results for the Difference in Capacity

\begin{tabular}{|c|c|c|c|c|c|c|c|c|c|c|c|c|}
\hline & \multirow[t]{4}{*}{ TWZ } & \multicolumn{2}{|c|}{ LMS } & \multicolumn{6}{|c|}{ CLMS } \\
\hline & & & & & \multirow{3}{*}{$\begin{array}{l}\sigma_{\text {Non- }} \\
C V\end{array}$} & \multirow{3}{*}{$\begin{array}{l}\sigma_{\text {Non- }} \\
C V \\
0.6\end{array}$} & \multirow{2}{*}{\multicolumn{3}{|c|}{$\begin{array}{c}F=0.2 \\
\psi\end{array}$}} & \multirow{2}{*}{\multicolumn{3}{|c|}{$\begin{array}{c}F=0.4 \\
\psi\end{array}$}} \\
\hline & & & & & & & & & & & & \\
\hline & & & & & & & 200 & 500 & 1000 & 200 & $\begin{array}{l}50 \\
0\end{array}$ & $\begin{array}{l}100 \\
0\end{array}$ \\
\hline \multicolumn{13}{|l|}{ TWZ } \\
\hline \multirow{2}{*}{ LMS } & \multicolumn{2}{|c|}{$\sigma_{\text {Non- } \mathrm{CV}}$} & 0.3 & $\begin{array}{l}0.00 \\
*\end{array}$ & & & & & & & & \\
\hline & \multicolumn{2}{|c|}{$\sigma_{\text {Non-CV }}$} & 0.6 & $\begin{array}{l}0.00 \\
*\end{array}$ & $\begin{array}{l}0.02 \\
*\end{array}$ & & & & & & & \\
\hline \multirow{6}{*}{$\begin{array}{l}\text { CLM } \\
\mathrm{S}\end{array}$} & \multirow{3}{*}{$\begin{array}{l}F \\
= \\
0 . \\
2\end{array}$} & $\psi$ & 200 & $\begin{array}{l}0.00 \\
*\end{array}$ & $\begin{array}{l}0.00 \\
*\end{array}$ & $\begin{array}{l}0.00 \\
*\end{array}$ & & & & & & \\
\hline & & $\psi$ & 500 & $\begin{array}{l}0.00 \\
*\end{array}$ & 0.14 & $\begin{array}{l}0.00 \\
*\end{array}$ & $\begin{array}{l}0.00 \\
*\end{array}$ & & & & & \\
\hline & & $\psi$ & $\begin{array}{l}100 \\
0\end{array}$ & $\begin{array}{l}0.00 \\
*\end{array}$ & 0.60 & 0.21 & $\begin{array}{l}0.00 \\
*\end{array}$ & $\begin{array}{l}0.00 \\
*\end{array}$ & & & & \\
\hline & \multirow{3}{*}{$\begin{array}{l}F \\
= \\
0 . \\
4\end{array}$} & $\psi$ & 200 & $\begin{array}{l}0.00 \\
*\end{array}$ & $\begin{array}{l}0.00 \\
*\end{array}$ & $\begin{array}{l}0.00 \\
*\end{array}$ & $\begin{array}{l}0.00 \\
*\end{array}$ & $\begin{array}{c}0.00 \\
*\end{array}$ & $\begin{array}{c}0.00 \\
*\end{array}$ & & & \\
\hline & & $\psi$ & 500 & $\begin{array}{l}0.00 \\
*\end{array}$ & $\begin{array}{l}0.00 \\
*\end{array}$ & $\begin{array}{l}0.00 \\
*\end{array}$ & $\begin{array}{l}0.00 \\
*\end{array}$ & $\begin{array}{c}0.00 \\
*\end{array}$ & $\begin{array}{c}0.00 \\
*\end{array}$ & $\begin{array}{l}0.2 \\
7\end{array}$ & & \\
\hline & & $\psi$ & $\begin{array}{l}100 \\
0\end{array}$ & $\begin{array}{l}0.00 \\
*\end{array}$ & $\begin{array}{l}0.00 \\
*\end{array}$ & $\begin{array}{l}0.00 \\
*\end{array}$ & $\begin{array}{l}0.00 \\
*\end{array}$ & $\begin{array}{c}0.00 \\
*\end{array}$ & $\begin{array}{c}0.00 \\
*\end{array}$ & $\begin{array}{l}0.5 \\
6\end{array}$ & $\begin{array}{l}0 . \\
8\end{array}$ & \\
\hline
\end{tabular}

*Significant

\begin{tabular}{|l|l|}
\hline There is no significant difference & \\
\hline The mean of sample in column is significantly higher than mean of sample in row & \\
\hline The mean of sample in row is significantly higher than mean of sample in column & \\
\hline
\end{tabular}

From T-test results, it appears that there is significant difference between all systems in terms of throughput. The CLMS shows significantly higher throughput than late merge 
system and traditional work zone as expected. By looking carefully at the comparison table, the CLMS when reduction factor is 0.2 is not significantly different from the late merge system with compliance rate of 0.6 . The effect of reduction factor is noticeable and the T-test results confirmed that there is significant difference between 0.2 and 0.4 . When the reduction factor is 0.2 , late merge area of 200 feet in the CLMS provides significantly higher capacity comparing to 500 and 1000 feet. When the reduction factor is 0.4 , longer late merge area can improve the capacity of the CLMS.

\subsubsection{Volume-to-Capacity (V/C)}

The V/C, volume-to-capacity, can provide an indication about the saturation of the segment. Traffic engineers commonly target a $\mathrm{V} / \mathrm{C}$ of 0.9 . Therefore, the traffic demand when $\mathrm{V} / \mathrm{C}=0.9$ was detected and investigated.

When $0.9 \mathrm{~V} / \mathrm{C}$ ratio is the independent variable, heavy vehicle percentage appears as a significant variable in the traditional work zone system. The following table summarizes the statistical test of traffic demands when $\mathrm{V} / \mathrm{C}=0.9$ in the traditional work zone system:

Table 25 Statistical Test for $0.9 \mathrm{~V} / \mathrm{C}$ in Traditional Work Zone

\begin{tabular}{|l|l|l|l|l|l|}
\hline Variable & Coef & SE Coef & T-Value & P-Value & VIF \\
\hline Constant & 1817 & 121 & 14.99 & 0.000 & \\
\hline$\varphi$ & -1558 & 431 & -3.62 & $0.011^{*}$ & 1.00 \\
\hline$\rho$ & -233 & 215 & -1.08 & 0.322 & 1.00 \\
\hline
\end{tabular}

* Significant Variable 
By considering the significant variables in regression analysis, the following regression model, with $\mathrm{R}^{2}=0.65$, describes throughput of late merge system:

$$
\Lambda=1701.1-1558 \varphi
$$

Where:

$$
\begin{aligned}
& \Lambda \text { is } 0.9 \mathrm{~V} / \mathrm{C} \text { of traditional work zone } \\
& \varphi \text { is heavy vehicle percentage }
\end{aligned}
$$

In late merge, however, traffic demands when $\mathrm{V} / \mathrm{C}=0.9$ is not explained by any of the considered variables. The following table summarizes the statistical test of $0.9 \mathrm{~V} / \mathrm{C}$ in the late merge system:

Table 26 Statistical Test for $0.9 \mathrm{~V} / \mathrm{C}$ in Late Merge System

\begin{tabular}{|l|l|l|l|l|l|}
\hline Variable & Coef & SE Coef & T-Value & P-Value & VIF \\
\hline Constant & 2398 & 134 & 17.93 & 0.000 & \\
\hline$\varphi$ & -3945 & 335 & -11.76 & 0.639 & 1.00 \\
\hline$\rho$ & -79 & 168 & -0.47 & 0.639 & 1.00 \\
\hline$\psi$ & 0.0735 & 0.0830 & 0.88 & 0.381 & 1.00 \\
\hline$\sigma_{\text {Non-CV }}$ & 340 & 183 & 1.86 & 0.068 & 1.00 \\
\hline
\end{tabular}

* Significant Variable

In the CLMS, heavy vehicle percentage, lane distribution and market penetration rate are significant variables. The market penetration rate increases the input traffic demand when $\mathrm{V} / \mathrm{C}=0.9$. In other words, the higher number of CVs improves the capacity of the 
CLMS. The following table summarizes the statistical test of traffic demands when V/C $=0.9$ in CLMS:

Table 27 Statistical Test for $0.9 \mathrm{~V} / \mathrm{C}$ in CLMS

\begin{tabular}{|l|l|l|l|l|l|}
\hline Variable & Coef & SE Coef & T-Value & P-Value & VIF \\
\hline Constant & 2392.0 & 58.6 & 40.83 & 0.000 & \\
\hline$\varphi$ & -3433 & 135 & -25.46 & $0.000^{*}$ & 1.00 \\
\hline$\rho$ & -163.4 & 67.4 & -2.42 & $0.016^{*}$ & 1.00 \\
\hline$\psi$ & -0.0822 & 0.0334 & -2.46 & 0.114 & 1.00 \\
\hline$\omega$ & 103.0 & 38.9 & 2.65 & $0.009^{*}$ & 1.00 \\
\hline$F$ & 119 & 110 & 1.08 & 0.280 & 1.00 \\
\hline
\end{tabular}

* Significant Variable

By considering the significant variables in regression analysis, the following regression model, with $\mathrm{R}^{2}=0.71$, describes throughput of late merge system:

$$
\Lambda=2309.7-3433 \varphi-163.4 \rho+103.0 \omega
$$

Where:
$\Lambda$ is $0.9 \mathrm{~V} / \mathrm{C}$ of CLMS
$\varphi$ is heavy vehicle percentage
$\rho$ is lane distribution
$\omega$ is market penetration rate

The following table summarizes the significant variables impacting traffic demand when $\mathrm{V} / \mathrm{C}=0.9$ in each system: 
Table 28 Significant Variables Impact $0.9 \mathrm{~V} / \mathrm{C}$ for All Systems

\begin{tabular}{|l|l|l|l|l|l|c|c|c|}
\hline System & \multicolumn{9}{|c|}{ Variable } \\
\hline Traditional Work Zone & $\gamma$ & $\rho^{*}$ & $\varphi$ & - & - & - & - & - \\
\hline Late Merge & $\gamma$ & $\rho$ & $\varphi$ & $\psi$ & $\sigma_{\text {Non-CV }}$ & - & - & - \\
\hline CLMS & $\gamma$ & $\rho^{*}$ & $\varphi^{*}$ & $\psi$ & $\sigma_{\text {Non-CV }}$ & $\sigma_{C V}$ & $F$ & $\omega^{*}$ \\
\hline
\end{tabular}

* Significant Variable

To compare the three system, only significant variables are taken into consideration.

However, heavy vehicle percentage is assumed as a reasonable value of $10 \%$ for the sake of conciseness. The following graph illustrates traffic volume of each system when 0.9 $\mathrm{V} / \mathrm{C}$ is achieved.

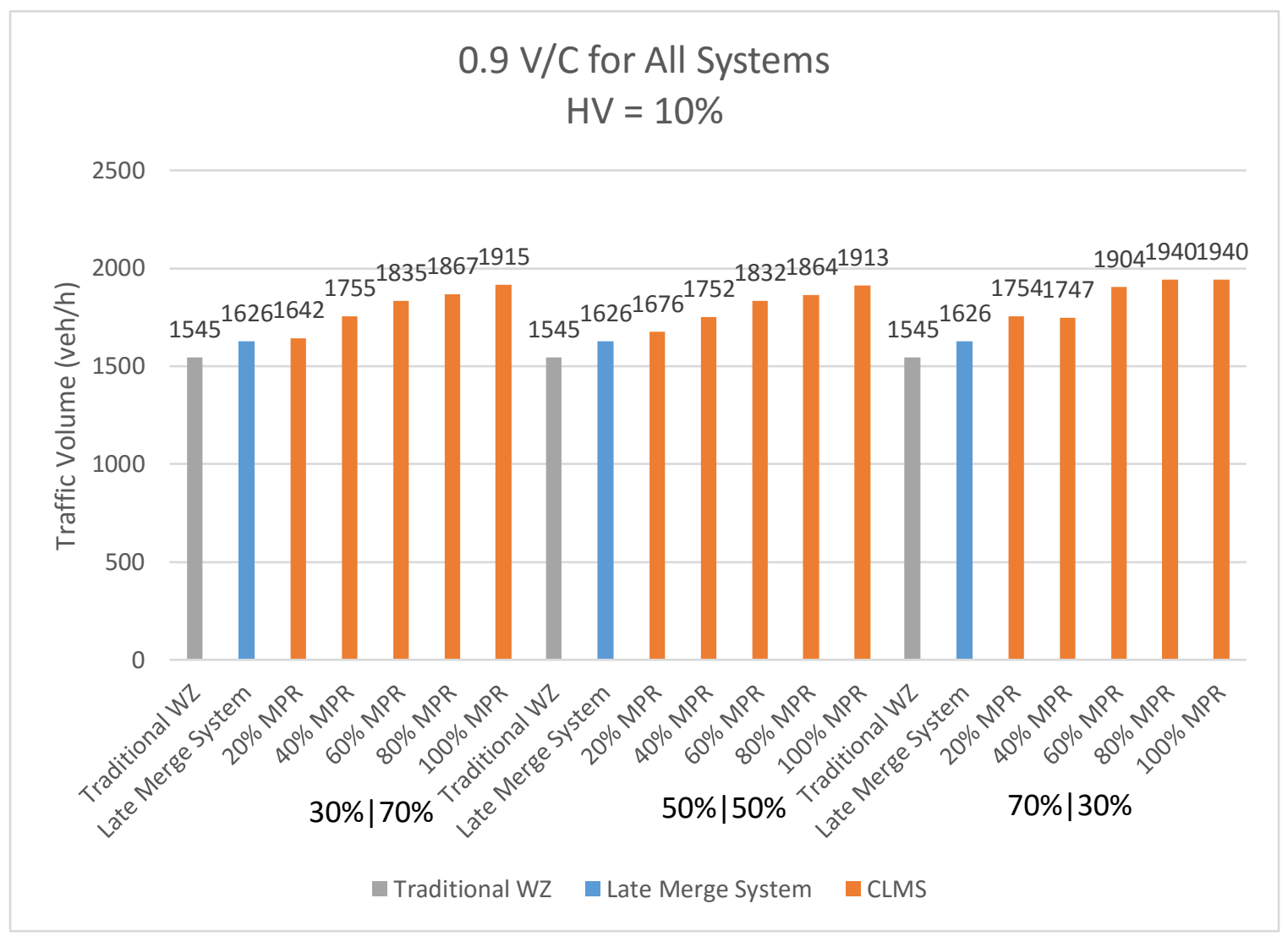

Figure 24 Comparison of $0.9 \mathrm{~V} / \mathrm{C}$ for All Systems 
As the graph shows, late merge system improved the traffic volume of $0.9 \mathrm{~V} / \mathrm{C}$ compared to the traditional work zone system. Ultimately, the CLMS shows a significant improvement with the increase of market penetration rate. Although, lane distribution is a significant variable, only $70 \% \mid 30 \%$ lane distribution prevented the improvement in 0.9 $\mathrm{V} / \mathrm{C}$ ratio with the increase of market penetration rates of $40 \%$ and $100 \%$.

It appears that the difference between the CLMS in case of $20 \%$ market penetration rate and late merge system is insignificant. However, in the scenarios of $40 \%$ and higher, the difference can be noticed.

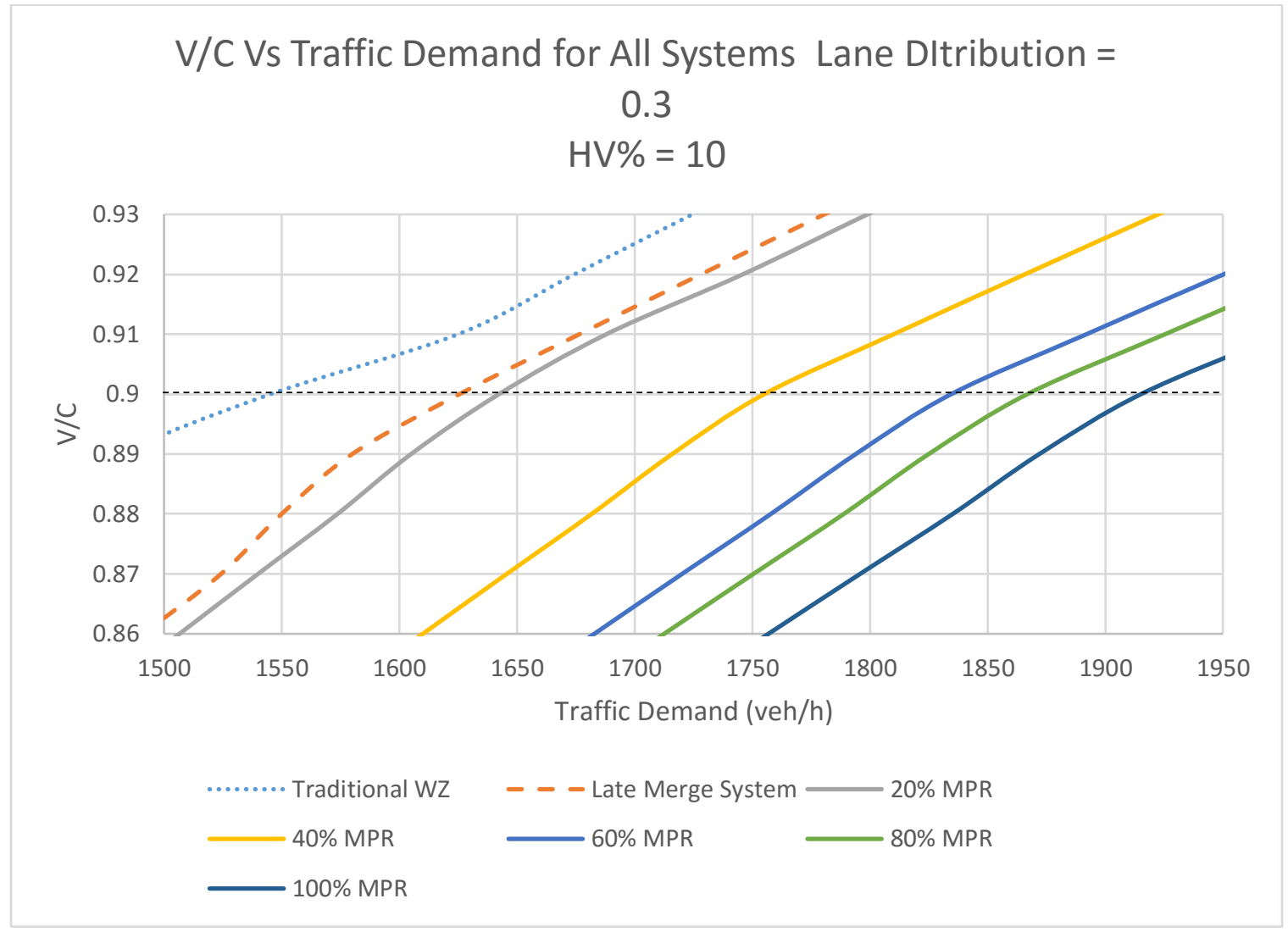

Figure 25 Comparison of V/C for All Systems When Lane Distribution is 30\% 


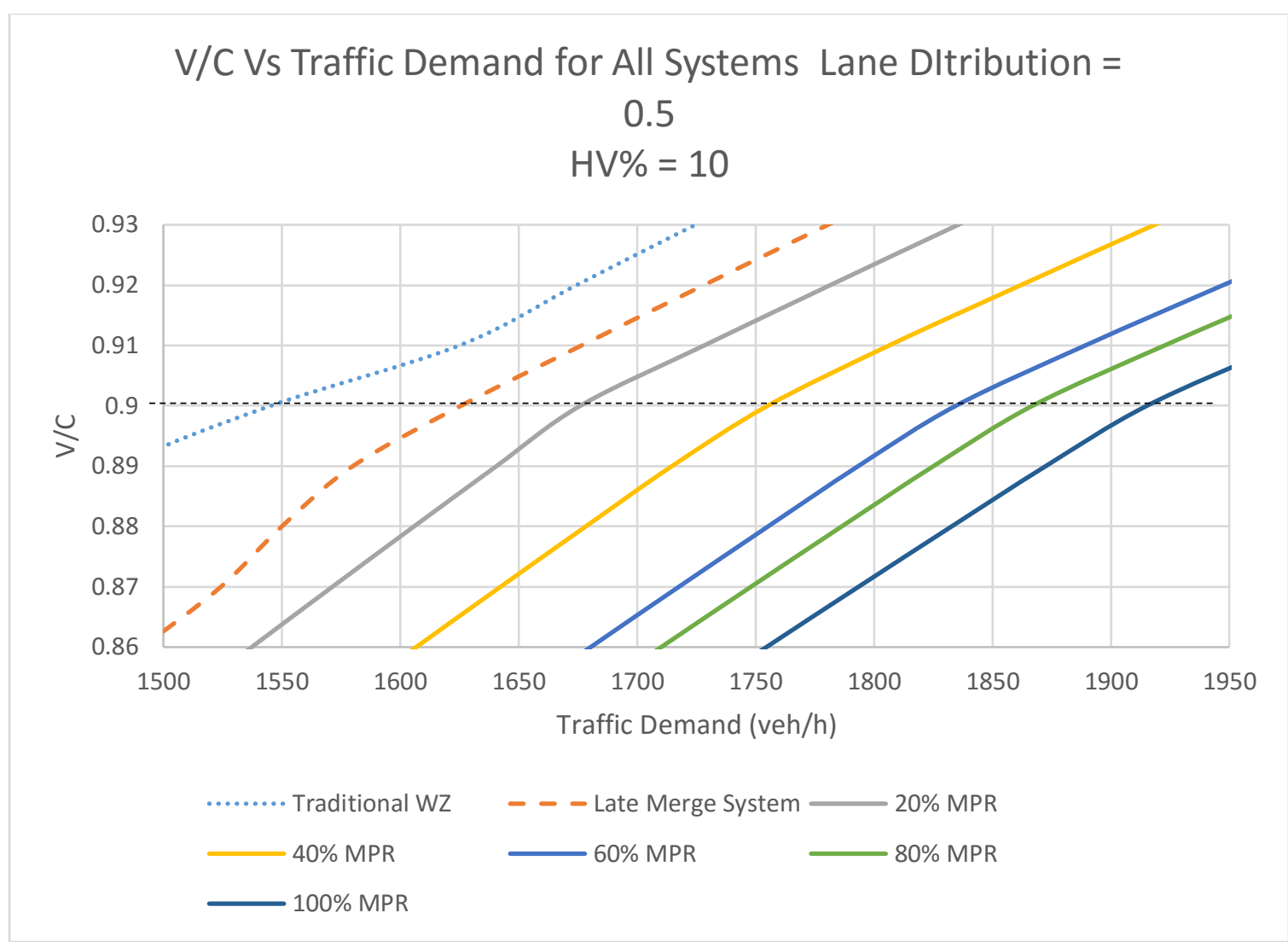

Figure 26 Comparison of V/C for All Systems When Lane Distribution is $50 \%$ 


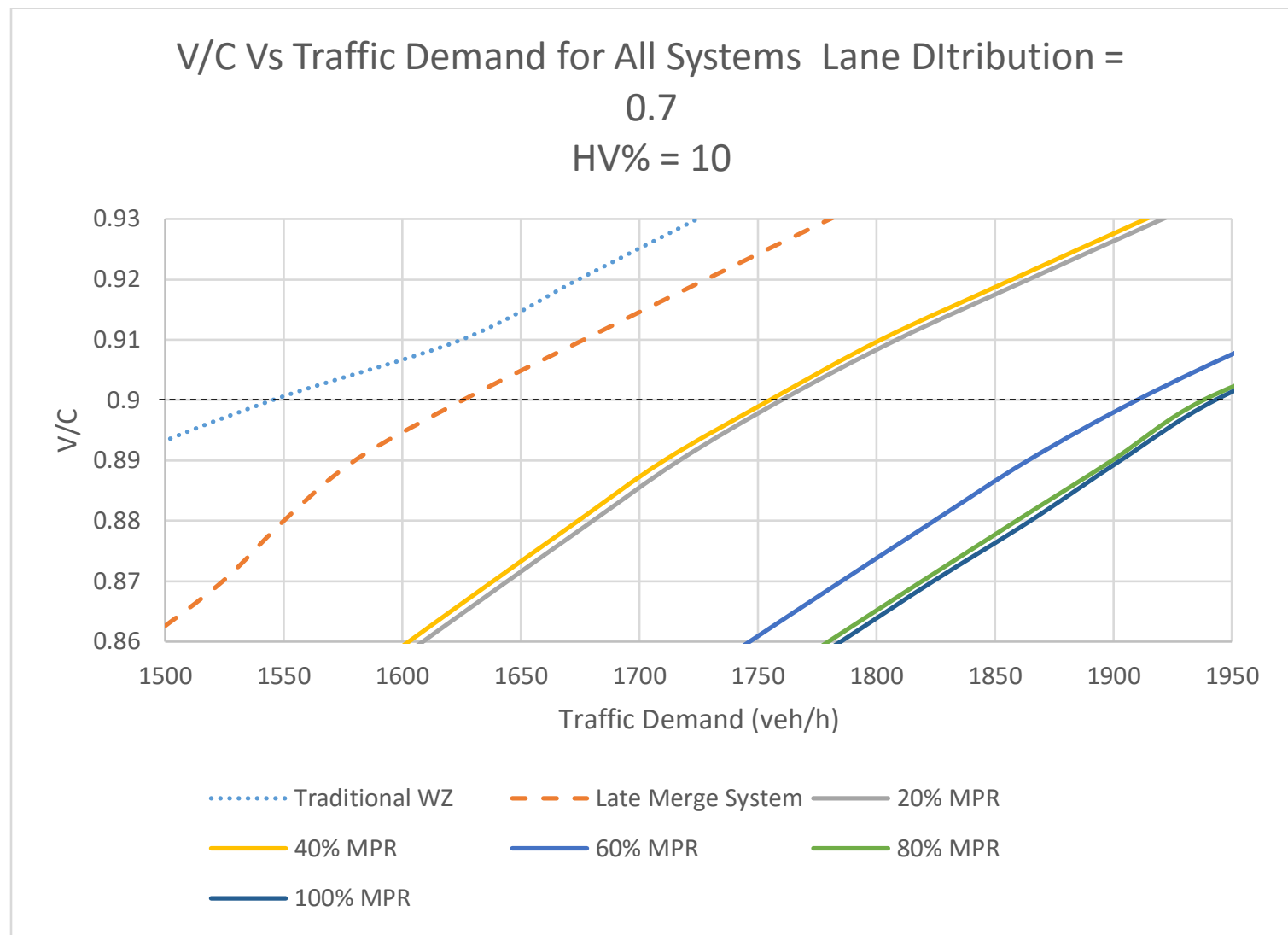

Figure 27 Comparison of V/C for All Systems When Lane Distribution is $70 \%$

From the previous three figures, it appears that lane distribution impacts the relation between market penetration rate and traffic demands when $\mathrm{V} / \mathrm{C}=0.9$. The lane distribution of 0.3 and 0.5 have a similar pattern, but 0.5 increases the demand of $0.9 \mathrm{~V} / \mathrm{C}$ when market penetration rate is $20 \%$. Lane distribution of 0.7 provides higher capacity in particular market penetration rates such as $20 \%$.

CLMS proved significant difference comparing to traditional work zone and late merge system. For concision, the following table shows t-test for the systems when lane distribution is 0.3 : 
Table 29 Values of $0.9 \mathrm{~V} / \mathrm{C}$ for Compared Systems

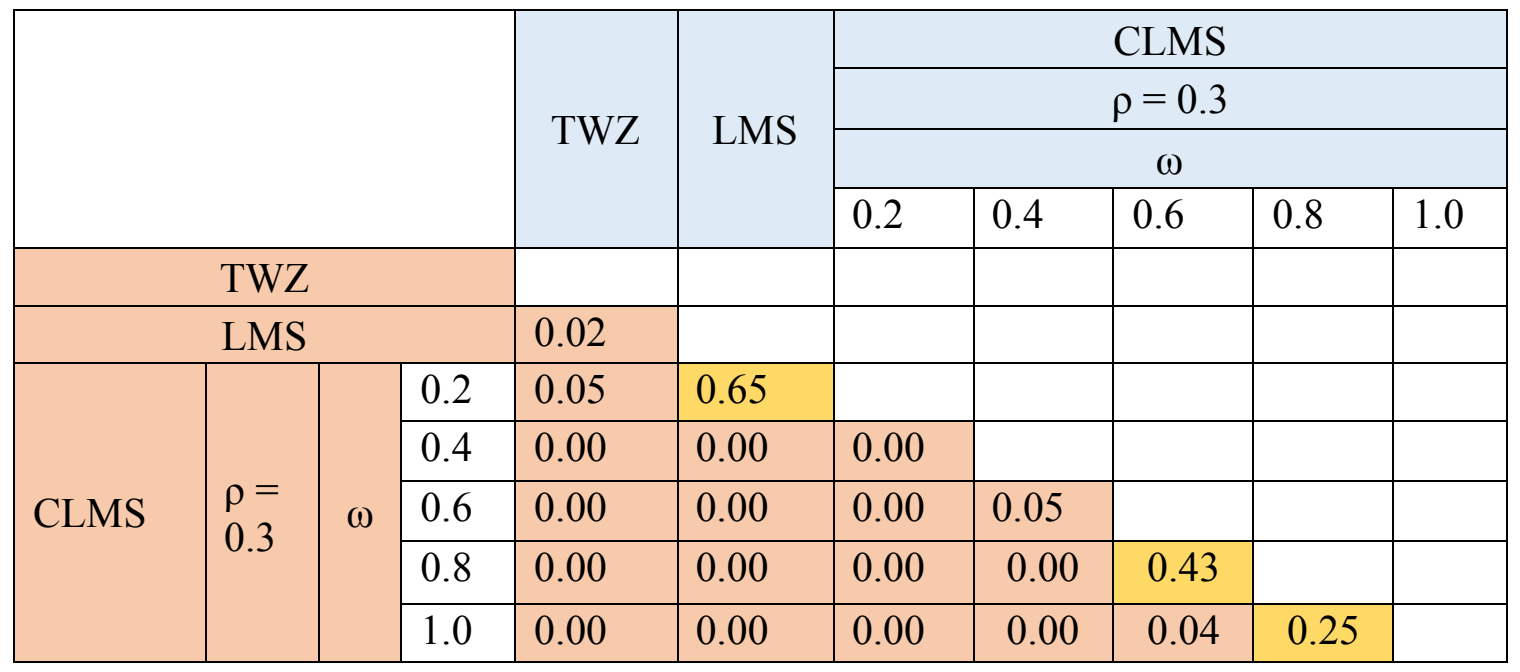

*Significant

\begin{tabular}{|l|l|}
\hline There is no significant difference & \\
\hline The mean of sample in column is significantly higher than mean of sample in row & \\
\hline The mean of sample in row is significantly higher than mean of sample in column & \\
\hline
\end{tabular}

From T-test results, it appears that there is significant difference between all systems in terms of throughput. The CLMS shows significantly higher throughput than late merge system and traditional work zone as expected. By looking carefully at the comparison table, the CLMS, when market penetration rate is $20 \%$, is not significantly different from the late merge system. However, other market penetration rates of CV in CLMS means significantly higher demand at $0.9 \mathrm{~V} / \mathrm{C}$ compared to late merge system. Also, there is no significant difference between market penetration rate of $60 \%$ and $80 \%$ as well as between $80 \%$ and $100 \%$. 


\subsubsection{Work Zone Delay}

Delay is measured by comparing the ideal time taken to drive a segment to the actual time. It is an indicator of the time spent in the work zone for each vehicle on average. Higher overall delay is a negative indication of the traffic operation of the work zone.

In traditional work zone, heavy vehicle percentage, and traffic demand are significant variables. Heavy vehicles need longer gap to merge which may result a longer wait at the taper. This waiting for a gap by heavy vehicles forces other vehicles on the closed lane to wait which increases the overall delay. The following table summarizes the statistical test of overall delay in traditional work zone system:

Table 30 Statistical Test for Delay in Traditional Work Zone

\begin{tabular}{|l|l|l|l|l|l|}
\hline Variable & Coef & SE Coef & T-Value & P-Value & VIF \\
\hline Constant & 213.99 & 6.60 & 32.44 & 0.000 & \\
\hline$\varphi$ & 134.1 & 17.1 & 7.83 & $0.000^{*}$ & 1.00 \\
\hline$\gamma$ & 0.00873 & 0.00205 & 4.27 & $0.000^{*}$ & 1.00 \\
\hline$\rho$ & -2.95 & 8.56 & -0.34 & 0.732 & 1.00 \\
\hline
\end{tabular}

* Significant Variable

By considering the significant variables in regression analysis, the following regression model, $\mathrm{R}^{2}=0.61$, describes delay of traditional work zone system:

$$
\Lambda=212.51+134.1 \varphi+0.00873 \gamma
$$

Where:

$\Lambda$ is delay of traditional work zone 
$\varphi$ is heavy vehicle percentage

$\gamma$ is traffic demand

In late merge system, heavy vehicle percentage, traffic demand and the compliance rate of non-CV are significant variable. It appears that higher compliance rate decreases the delay as more vehicles apply late merge, which is beneficial to the system. The following table summarizes the statistical test of overall delay in late merge system:

Table 31 Statistical Test for Delay in Late Merge System

\begin{tabular}{|l|l|l|l|l|l|}
\hline Variable & Coef & SE Coef & T-Value & P-Value & VIF \\
\hline Constant & 5.20 & 4.44 & 1.17 & 0.242 & \\
\hline$\varphi$ & 188.55 & 9.29 & 20.29 & $0.000^{*}$ & 1.00 \\
\hline$\gamma$ & 0.01140 & 0.00111 & 10.27 & $0.000^{*}$ & 1.00 \\
\hline$\rho$ & 5.75 & 4.65 & 1.24 & 0.217 & 1.00 \\
\hline$\psi$ & 0.00257 & 0.00230 & 1.12 & 0.264 & 1.00 \\
\hline$\sigma_{\text {Non-CV }}$ & -36.49 & 5.06 & -7.21 & $0.000^{*}$ & 1.00 \\
\hline
\end{tabular}

* Significant Variable

By considering the significant variables in regression analysis, the following regression model, with $\mathrm{R}^{2}=0.47$, describes delay of traditional work zone system:

$$
\Lambda=-6.78+258.5 \varphi+0.02476 \gamma+53.2 \sigma_{\text {Non-CV }}
$$

Where:

$\Lambda$ is delay in late merge system

$\varphi$ is heavy vehicle percentage

$\gamma$ is traffic demand

$\sigma_{\text {Non-CV }}$ is compliance rate for non-CV 
With heavy vehicle percentage and traffic demand, late merge area and reduction factor are significant variables in the delay of CLMS. When the late merge area is small, lower delay was observed. The following table summarizes the statistical test of overall delay in the CLMS:

Table 32 Statistical Test for Delay in CLMS

\begin{tabular}{|l|l|l|l|l|l|}
\hline Variable & Coef & SE Coef & T-Value & P-Value & VIF \\
\hline Constant & -41.34 & 7.75 & -5.34 & 0.000 & \\
\hline$\varphi$ & 327.9 & 15.3 & 21.49 & $0.000^{*}$ & 1.00 \\
\hline$\gamma$ & 0.01622 & 0.00182 & 8.89 & $0.000^{*}$ & 1.00 \\
\hline$\rho$ & 7.91 & 7.63 & 1.04 & 0.300 & 1.00 \\
\hline$\psi$ & -0.04503 & 0.00377 & 11.93 & $0.000^{*}$ & 1.00 \\
\hline$\omega$ & 2.12 & 4.40 & 0.48 & 0.630 & 1.00 \\
\hline$F$ & 158.3 & 12.5 & 12.70 & $0.000^{*}$ & 1.00 \\
\hline
\end{tabular}

* Significant Variable

By considering the significant variables in regression analysis, the following regression model, with $\mathrm{R}^{2}=0.34$, describes delay of traditional work zone system:

$$
\Lambda=-37.53+327.9 \varphi+0.01687 \gamma+0.04532 \psi+157.4 F
$$

Where:

$\Lambda$ is delay in CLMS

$\varphi$ is heavy vehicle percentage

$\gamma$ is traffic demand

$\psi$ is late merge area

$F$ is reduction factor for gap acceptance 
The following table summarizes the significant variables affecting overall delay in each system:

Table 33 Significant Variables Impact Delay for All Systems

\begin{tabular}{|l|l|l|l|l|l|c|c|c|}
\hline System & \multicolumn{9}{|c|}{ Variable } \\
\hline Traditional Work Zone & $\gamma^{*}$ & $\rho$ & $\varphi^{*}$ & - & - & - & - & - \\
\hline Late Merge & $\gamma^{*}$ & $\rho$ & $\varphi^{*}$ & $\psi$ & $\sigma_{\text {Non-CV }}$ & - & - & - \\
\hline CLMS & $\gamma^{*}$ & $\rho$ & $\varphi^{*}$ & $\psi^{*}$ & $\sigma_{\text {Non-CV }}$ & $\sigma_{C V}$ & $F^{*}$ & $\omega$ \\
\hline
\end{tabular}

* Significant Variable

To compare the three system, only significant variables are taken into consideration.

However, heavy vehicle percentage is assumed as a reasonable value of $10 \%$ for the sake of conciseness. The effect of compliance rate for non-CV will be discussed later. The following graph illustrates the delay of the three systems with the significant variables. 


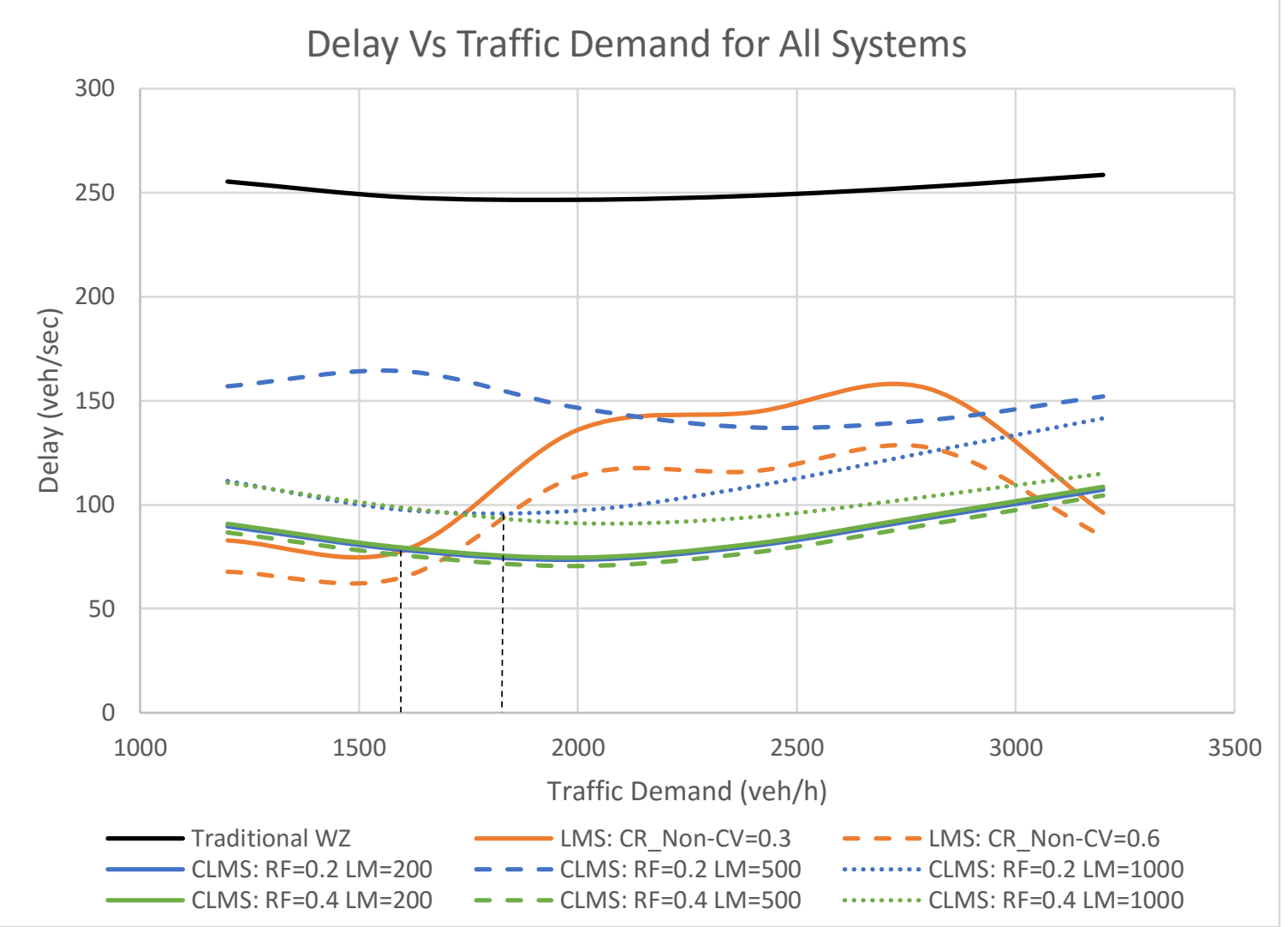

Figure 28 Comparison of Delay for All Systems

As appear in figure 28, the CLMS has a general improved delay compared to traditional work zone and late merge system. The CLMS and late merge system started performing similarly at low traffic demand. Late merge system showed higher delay at moderate traffic demands and oddly improved at higher traffic demand. This can be related to the saturation level of the two lanes as at a certain level of saturation, late merge system is unable to perform well. The effect of the reduction factor appear in the CLMS (500 feet) as the delay was substantially improved when it is 0.4 . This can be visually seen around the traffic demand of $1800 \mathrm{veh} / \mathrm{h}$. Moreover, 0.4 reduction factor provides lower delay in the three CLMS late merge areas on average. 
The T-test results shows significant difference between late merge system and the CLMS. The following table summarizes T-test results:

Table 34 T-Test Results for the Difference of Delay

\begin{tabular}{|c|c|c|c|c|c|c|c|c|c|c|c|c|}
\hline & \multirow[t]{4}{*}{ TWZ } & \multicolumn{2}{|c|}{ LMS } & \multicolumn{6}{|c|}{ CLMS } \\
\hline & & & & & \multirow{3}{*}{$\begin{array}{l}\sigma_{\text {Non- }} \\
\mathrm{CV} \\
0.3\end{array}$} & \multirow{3}{*}{$\begin{array}{l}\sigma_{\text {Non- }} \\
\mathrm{CV} \\
0.6\end{array}$} & \multicolumn{3}{|c|}{$\mathrm{F}=0.2$} & \multicolumn{3}{|c|}{$F=0.4$} \\
\hline & & & & & & & \multicolumn{3}{|c|}{$\psi$} & \multicolumn{3}{|c|}{$\psi$} \\
\hline & & & & & & & 200 & 500 & 1000 & 200 & 500 & 1000 \\
\hline \multicolumn{13}{|l|}{ TWZ } \\
\hline \multirow{2}{*}{ LMS } & \multicolumn{2}{|c|}{$\begin{array}{l}\sigma_{\text {Non- }} \\
\mathrm{CV}\end{array}$} & 0.3 & 0.00 & & & & & & & & \\
\hline & \multicolumn{2}{|c|}{$\begin{array}{l}\sigma_{\text {Non- }} \\
\mathrm{CV}\end{array}$} & 0.6 & 0.00 & 0.00 & & & & & & & \\
\hline \multirow{6}{*}{ CLMS } & \multirow{3}{*}{$\begin{array}{l}\mathrm{F} \\
= \\
0.2\end{array}$} & \multirow{3}{*}{$\psi$} & 200 & 0.00 & 0.00 & 0.02 & & & & & & \\
\hline & & & 500 & 0.00 & 0.00 & 0.00 & 0.02 & & & & & \\
\hline & & & 1000 & 0.00 & 0.00 & 0.04 & 0.00 & 0.00 & & & & \\
\hline & \multirow{3}{*}{$\begin{array}{l}F \\
= \\
0.4\end{array}$} & \multirow{3}{*}{$\psi$} & 200 & 0.00 & 0.00 & 0.60 & 0.06 & 0.00 & 0.00 & & & \\
\hline & & & 500 & 0.00 & 0.00 & 0.00 & 0.00 & 0.00 & 0.00 & 0.00 & & \\
\hline & & & 1000 & 0.00 & 0.91 & 0.00 & 0.00 & 0.00 & 0.00 & 0.00 & 0.00 & \\
\hline
\end{tabular}

*Significant

\begin{tabular}{|l|}
\hline There is no significant difference \\
\hline The mean of sample in column is significantly higher than mean of sample in row \\
\hline The mean of sample in row is significantly higher than mean of sample in column \\
\hline
\end{tabular}

From T-test results, it appears that there is significant difference between all systems in terms of delay. The CLMS shows significantly lower delay than late merge system and traditional work zone as expected. By looking carefully at the comparison table, the CLMS, when reduction factor is 0.4 and late merge area is 1000 feet, the CLMS is not significantly different from late merge system. When late merge area is 500 feet and the reduction factor is 0.4 , late merge system is even better than the CLMS, while other late merge areas have significantly lower delay. 


\subsubsection{Work Zone Queue Length}

Queue length is a measurement of the length, in feet, of traffic backup that caused by the bottleneck. One of the major objectives of late merge strategy is to shorten the queue length by filling up the empty space in the closed lane.

In traditional work zone, heavy vehicle percentage, and traffic demand are significant variables in the queue length as found previously in the overall delay. The following table summarizes the statistical test of overall delay in traditional work zone system:

Table 35 Statistical Test for Queue Length in Traditional Work Zone

\begin{tabular}{|l|l|l|l|l|l|}
\hline Variable & Coef & SE Coef & T-Value & P-Value & VIF \\
\hline Constant & 1673.81 & 0.02 & 93471.60 & 0.000 & \\
\hline$\varphi$ & -0.1798 & 0.0465 & -3.87 & $0.000^{*}$ & 1.00 \\
\hline$\gamma$ & 0.000013 & 0.000006 & 2.33 & $0.024^{*}$ & 1.00 \\
\hline$\rho$ & 0.0019 & 0.0232 & 0.08 & 0.935 & 1.00 \\
\hline
\end{tabular}

* Significant Variable

By considering the significant variables in regression analysis, the following regression model, with $\mathrm{R}^{2}=0.28$, describes throughput of late merge system:

$$
\Lambda=1673.81-0.1798 \varphi+0.000013 \gamma
$$

Where:

$\Lambda$ is queue length of traditional work zone $\varphi$ is heavy vehicle percentage $\gamma$ is traffic demand 
In addition to heavy vehicle percentage and traffic demand, the compliance rate of non$\mathrm{CV}$ is also significant variable in late merge system. Again, higher compliance rate decreases the delay as more vehicles apply late merge, which distributes the traffic on the two lane and reduces the length of the open lane. The following table summarizes the statistical test of queue length in late merge system:

Table 36 Statistical Test for Queue Length in Late Merge System

\begin{tabular}{|l|l|l|l|l|l|}
\hline Variable & Coef & SE Coef & T-Value & P-Value & VIF \\
\hline Constant & -161.7 & 59.1 & -2.74 & 0.007 & \\
\hline$\varphi$ & 1537 & 124 & 12.42 & $0.000^{*}$ & 1.00 \\
\hline$\gamma$ & 0.1139 & 0.0148 & 7.70 & $0.000^{*}$ & 1.00 \\
\hline$\rho$ & 61.7 & 61.9 & 1.00 & 0.319 & 1.00 \\
\hline$\psi$ & -0.0052 & 0.0306 & -0.17 & 0.865 & 1.00 \\
\hline$\sigma_{\text {Non-CV }}$ & -1458.0 & 67.4 & -21.64 & $0.000^{*}$ & 1.00 \\
\hline
\end{tabular}

* Significant Variable

By considering the significant variables in regression analysis, the following regression model, with $\mathrm{R}^{2}=0.68$, describes throughput of late merge system:

$$
\Lambda=-175.4+1596 \varphi+0.1357 \gamma+1537.3 \sigma_{\text {Non-CV }}
$$

Where:

$\Lambda$ is queue length of late merge system

$\varphi$ is heavy vehicle percentage

$\gamma$ is traffic demand

$\sigma_{\text {Non-CV }}$ is compliance rate for non-CV 
With heavy vehicle percentage and traffic demand, late merge area and reduction factor are significant variables in the delay of CLMS. When the late merge area is small, lower queue length was observed. The following table summarizes the statistical test of queue length in the CLMS:

Table 37 Statistical Test for Queue Length in CLMS

\begin{tabular}{|l|l|l|l|l|l|}
\hline Variable & Coef & SE Coef & T-Value & P-Value & VIF \\
\hline Constant & -122.3 & 40.9 & -2.99 & 0.003 & \\
\hline$\varphi$ & 2675.1 & 80.5 & 33.25 & $0.000^{*}$ & 1.00 \\
\hline$\gamma$ & 0.15518 & 0.00962 & 16.14 & $0.000^{*}$ & 1.00 \\
\hline$\rho$ & 79.4 & 40.2 & 1.97 & 0.049 & 1.00 \\
\hline$\psi$ & 0.2206 & 0.0199 & 11.08 & $0.000^{*}$ & 1.00 \\
\hline$\omega$ & 11.6 & 23.2 & 0.50 & 0.619 & 1.00 \\
\hline$F$ & 597.2 & 65.7 & 9.09 & $0.000^{*}$ & 1.00 \\
\hline
\end{tabular}

* Significant Variable

By considering the significant variables in regression analysis, the following regression model, with $\mathrm{R}^{2}=0.49$, describes throughput of late merge system:

$$
\Lambda=+2785.7 \varphi+0.3006 \gamma+0.2024 \psi-599.5 F-479.8
$$

Where:

$\Lambda$ is queue length in of CLMS

$\varphi$ is heavy vehicle percentage

$\gamma$ is traffic demand

$\psi$ is late merge area

$F$ is reduction factor for gap acceptance 
The following table summarizes the significant variables affecting the queue length in each system:

Table 38 Significant Variables Impact Queue Length for All Systems

\begin{tabular}{|l|l|l|l|l|l|l|l|c|}
\hline System & \multicolumn{9}{|c|}{ Variable } \\
\hline Traditional Work Zone & $\gamma^{*}$ & $\rho$ & $\varphi^{*}$ & - & - & - & - & - \\
\hline Late Merge & $\gamma^{*}$ & $\rho$ & $\varphi^{*}$ & $\psi$ & $\sigma_{\text {Non-CV }}$ & - & - & - \\
\hline CLMS & $\gamma$ & $\rho$ & $\varphi^{*}$ & $\psi^{*}$ & $\sigma_{\text {Non-CV }}$ & $\sigma_{C V}$ & $F^{*}$ & $\omega$ \\
\hline
\end{tabular}

* Significant Variable

To compare the three system, only significant variables are taken into consideration.

However, heavy vehicle percentage is assumed as a reasonable value of $10 \%$ for the sake of conciseness. The effect of compliance rate for non-CV will be discussed later. The following graph illustrates operations of the three systems with the significant variables: 


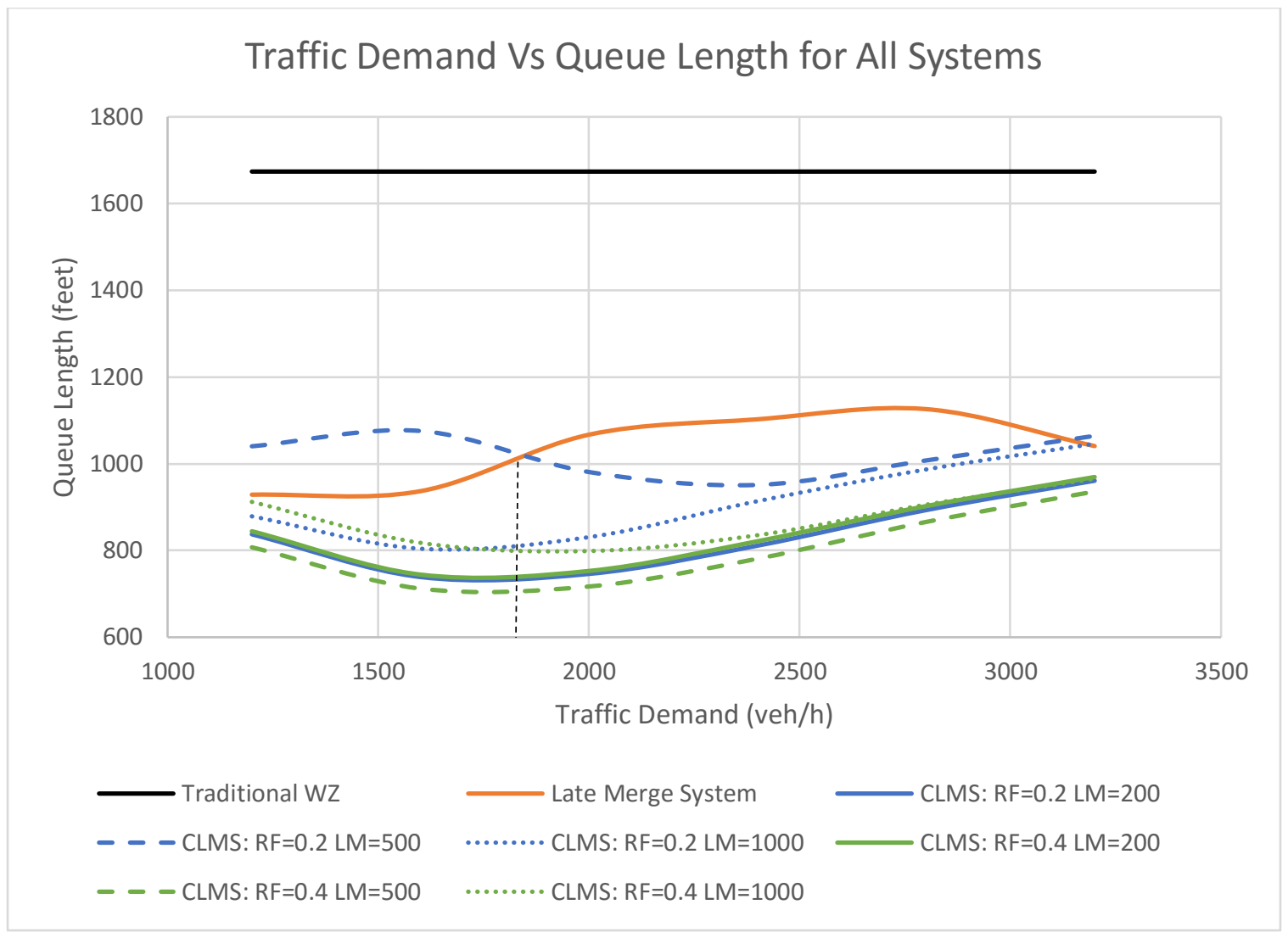

Figure 29 Comparison of Queue Length for All Systems

The relations between significant variables and the queue length follow the same pattern in the overall delay. As appear in figure 29, the performance of traditional work zone was steady because the queue extended till the end of simulation network during all simulation scenarios. The effect of the reduction factor appear in the CLMS (500 feet) as the queue length was substantially improved when it is 0.4 . This can be visually seen around the traffic demand of $1800 \mathrm{veh} / \mathrm{h}$. Moreover, 0.4 reduction factor provides lower queue length in the three CLMS systems on average. By comparing the CLMS with the late merge system, it appears that CLMS operates with lower queue length in all traffic demands. 
The t-test results showed significant difference between late merge system and the CLMS. The following table summarizes t-test results

Table 39 T-Test Results for the Difference of Queue Length

\begin{tabular}{|c|c|c|c|c|c|c|c|c|c|c|c|c|}
\hline & & \multirow{3}{*}{$\begin{array}{l}\sigma_{\text {Non- }}- \\
\mathrm{CV} \\
0.3\end{array}$} & \multirow{3}{*}{$\begin{array}{l}\sigma_{\text {Non- }} \\
\mathrm{CV} \\
0.6\end{array}$} & \multicolumn{3}{|c|}{$F=0.2$} & \multicolumn{3}{|c|}{$F=0.4$} \\
\hline & & & & & & & \multicolumn{3}{|c|}{$\psi$} & \multicolumn{3}{|c|}{$\psi$} \\
\hline & & & & & & & 200 & 500 & 1000 & 200 & 500 & 1000 \\
\hline \multicolumn{13}{|l|}{ TWZ } \\
\hline \multirow{2}{*}{ LMS } & \multicolumn{2}{|c|}{$\begin{array}{l}\sigma_{\text {Non- }} \\
C V\end{array}$} & 0.3 & 0.00 & & & & & & & & \\
\hline & \multicolumn{2}{|c|}{$\begin{array}{l}\sigma_{\text {ONon- }} \\
\mathrm{CV}\end{array}$} & 0.6 & 0.00 & 0.00 & & & & & & & \\
\hline \multirow{6}{*}{ CLMS } & \multirow{3}{*}{$\begin{array}{l}F \\
= \\
0.2\end{array}$} & $\psi$ & 200 & 0.00 & 0.00 & 0.02 & & & & & & \\
\hline & & $\psi$ & 500 & 0.00 & 0.00 & 0.46 & 0.00 & & & & & \\
\hline & & $\psi$ & 1000 & 0.00 & 0.00 & 0.00 & 0.00 & 0.00 & & & & \\
\hline & \multirow{3}{*}{$\begin{array}{l}\mathrm{F} \\
= \\
0.4\end{array}$} & $\psi$ & 200 & 0.00 & 0.00 & 0.50 & 0.15 & 0.82 & 0.00 & & & \\
\hline & & $\psi$ & 500 & 0.01 & 0.00 & 0.00 & 0.00 & 0.00 & 0.00 & 0.00 & & \\
\hline & & $\psi$ & 1000 & 0.00 & 0.00 & 0.00 & 0.00 & 0.03 & 0.01 & 0.00 & 0.00 & \\
\hline
\end{tabular}

*Significant

\section{There is no significant difference}

The mean of sample in column is significantly higher than mean of sample in row The mean of sample in row is significantly higher than mean of sample in column

From T-test results, it appears that there is significant difference between all systems in terms of queue length. The CLMS shows significantly lower queue length than late merge system and traditional work zone as expected. By looking carefully at the comparison table, the CLMS, when reduction factor is 0.4 and late merge area is 1000 feet, the CLMS is not significantly different from late merge system. When late merge area is 500 feet and the reduction factor is 0.4 , late merge system is even better than the CLMS, while other late merge areas have significantly lower delay. 


\subsection{Effect of Compliance Rate}

As mentioned earlier, compliance rate plays a significant role in applying new systems and due to the excessive number of experiments, the effect of compliance rate of CV and non-CV are investigated separately in this subsection. The scenarios consider only traffic demands and compliance rates of $\mathrm{CV}$ and Non-CV while other variable were fixed on certain values. The following table summarizes the variables and parameters in compliance rate effect experiment:

Table 40 Variables Considered in Compliance Rate Effect

\begin{tabular}{|l|l|}
\hline Variable & Value \\
\hline$\gamma$ & $1200 \mathrm{veh} / \mathrm{h}-3200 \mathrm{veh} / \mathrm{h}$ \\
\hline$\rho$ & $50 \% \mid 50 \%$ \\
\hline$\varphi$ & $10 \%$ \\
\hline$\psi$ & 200 feet \\
\hline$\sigma_{C V}$ & $50 \%-100 \%$ \\
\hline$\sigma_{\text {Non-CV }}$ & $30 \%$ and $60 \%$ \\
\hline F & $40 \%$ \\
\hline$\omega$ & $60 \%$ \\
\hline
\end{tabular}

Compliance rate of both $\mathrm{CV}$ and non-CV is a significant variable in throughput, delay and queue length. The following table summarizes the statistical test of compliance rates in the CLMS: 
Table 41 Significant Variables Impact Compliance Rate on CLMS

\begin{tabular}{|c|c|c|c|c|c|c|}
\hline & Variable & Coef & SE Coef & T-Value & P-Value & VIF \\
\hline \multirow{4}{*}{ Throughput } & Constant & 1016.3 & 44.6 & 22.81 & 0.000 & \\
\hline & $\gamma$ & 0.1854 & 0.0104 & 17.9 & $0.000^{*}$ & \\
\hline & $\sigma_{\mathrm{CV}}$ & 204.3 & 47.2 & 4.33 & $0.000^{*}$ & 1.00 \\
\hline & $\sigma_{\text {Non-CV }}$ & 65.4 & 41.4 & 1.58 & $0.019^{*}$ & 1.00 \\
\hline \multirow{4}{*}{ Capacity } & Constant & 1619 & 88.9 & 18.2 & 0.000 & \\
\hline & $\gamma$ & - & - & - & - & - \\
\hline & $\sigma_{\mathrm{CV}}$ & 124 & 110 & 1.13 & $0.042^{*}$ & 1.00 \\
\hline & $\sigma_{\text {Non-CV }}$ & 218.6 & 96.2 & 2.27 & $0.021 *$ & 1.00 \\
\hline \multirow{4}{*}{$0.9 \mathrm{~V} / \mathrm{C}$} & Constant & 2379 & 221 & 10.74 & 0.000 & \\
\hline & $\gamma$ & - & - & - & - & - \\
\hline & $\sigma_{\mathrm{CV}}$ & -311 & 230 & -1.35 & 0.226 & 1.04 \\
\hline & $\sigma_{\text {Non-CV }}$ & -688 & 261 & -2.64 & 0.391 & 1.04 \\
\hline \multirow{4}{*}{ Delay } & Constant & -34.8 & 14.5 & -2.4 & 0.019 & \\
\hline & $\gamma$ & 0.04308 & 0.00336 & 12.81 & $0.000^{*}$ & \\
\hline & $\sigma_{\mathrm{CV}}$ & 65.3 & 15.3 & 4.26 & $0.000^{*}$ & 1.00 \\
\hline & $\sigma_{\text {Non-CV }}$ & 17.5 & 13.5 & 1.3 & $0.017^{*}$ & 1.00 \\
\hline \multirow{4}{*}{$\begin{array}{l}\text { Queue } \\
\text { Length }\end{array}$} & Constant & -241 & 110 & -2.19 & 0.032 & \\
\hline & $\gamma$ & 0.03234 & 0.0256 & 12.65 & $0.000^{*}$ & 1.00 \\
\hline & $\sigma_{\mathrm{CV}}$ & 464 & 116 & 3.99 & $0.000^{*}$ & 1.00 \\
\hline & $\sigma_{\text {Non-CV }}$ & 133 & 102 & 1.30 & $0.018^{*}$ & 1.00 \\
\hline
\end{tabular}

* Significant Variable

By considering the significant variables in regression analysis, the following regression models with R-Squared values, describes the MOEs of CLMS in terms of compliance rates of $\mathrm{CV}$ and Non-CV:

$$
\Lambda=1016.3+0.1854 \gamma+204.3 \sigma_{C V}+65.4 \sigma_{\text {Non-CV }}
$$

Where:

$\Lambda$ is throughput in CLMS

$\sigma_{C V}$ is compliance rate for $\mathrm{CV}$ 
$\sigma_{\text {Non-CV }}$ is compliance rate for non-CV

$\mathrm{R}^{2}=0.82$

$\Lambda=1619.0+124 \sigma_{C V}+218.6 \sigma_{N o n-C V}$

Where:

$\Lambda$ is capacity in CLMS

$\sigma_{C V}$ is compliance rate for $\mathrm{CV}$

$\sigma_{\text {Non-CV }}$ is compliance rate for non-CV

$\mathrm{R}^{2}=0.41$

$\Lambda=-34.8+0.04308 \gamma+65.3 \sigma_{C V}+17.5 \sigma_{\text {Non-CV }}$

Where:

$\Lambda$ is delay in of CLMS

$\sigma_{C V}$ is compliance rate for $\mathrm{CV}$

$\sigma_{N o n-C V}$ is compliance rate for non-CV

$\mathrm{R}^{2}=0.73$

$\Lambda=-241+0.3234 \gamma+464 \sigma_{C V}+133 \sigma_{\text {Non-CV }}$

Where:

$\Lambda$ is queue length in of CLMS

$\sigma_{C V}$ is compliance rate for $\mathrm{CV}$

$\sigma_{\text {Non-CV }}$ is compliance rate for non-CV

$\mathrm{R}^{2}=0.72$ 
To visually determine the effect of compliance rate, the following graphs of throughput, capacity, $\mathrm{V} / \mathrm{C}$, delay and queue length for two levels of non-CV compliance rates and five levels of CV compliance rates are plotted:

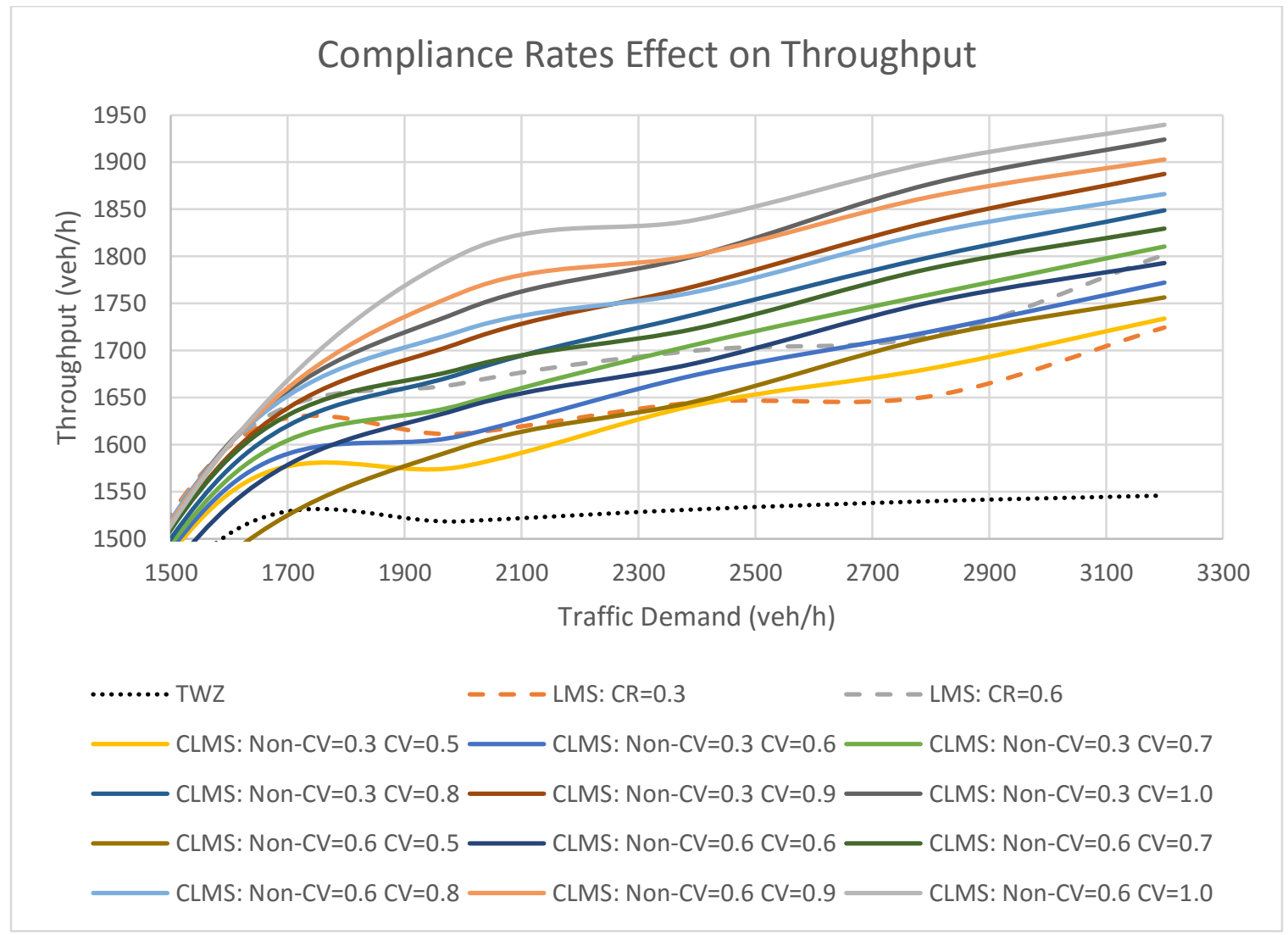

Figure 30 CV Compliance Rate Effect on Throughput in CLMS

As figure 30 illustrates, the increase of compliance rate in the CLMS leads to an increase of throughput. At lower traffic demand, all systems with different compliance rates operated similarly. When the compliance rate increase, the graph shows increase in throughput in both late merge system and the CLMS. T-test was conducted to test the 
significance of the difference between compliance rates. The following tables summarize t-test results:

Table 42 T-Test of Compliance Rate Effect on Throughput When $\sigma_{\text {Non-CV }}=0.3$

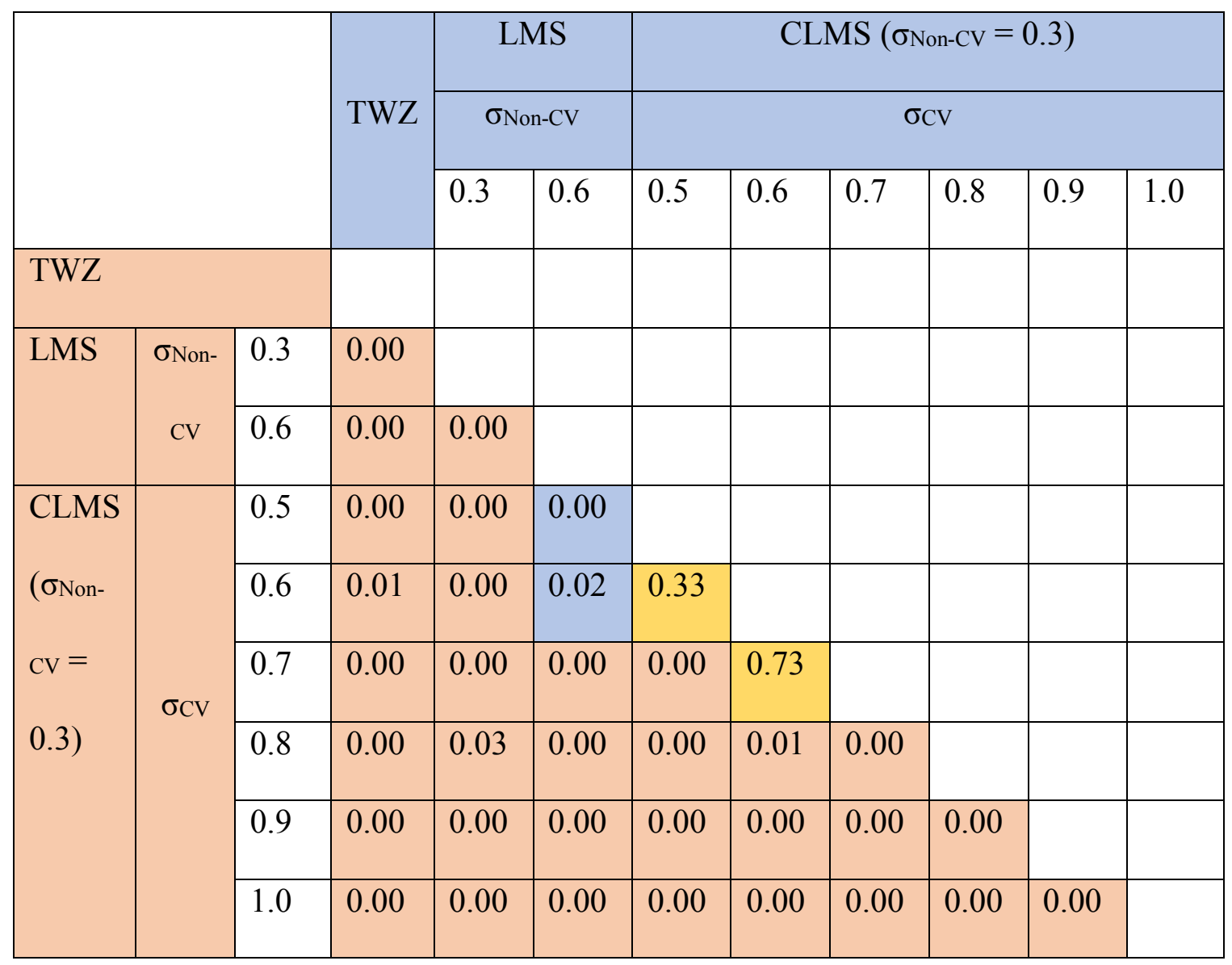

*Significant

There is no significant difference

The mean of sample in column is significantly higher than mean of sample in row

The mean of sample in row is significantly higher than mean of sample in column

As the table shows, throughput increases significantly with the growing of compliance rate in most cases. There is no significant difference between $50 \%$ and $60 \%$ as well as between $60 \%$ and $70 \%$ in the CLMS. 
Table 43 T-Test of Compliance Rate Effect on Throughput When $\sigma$ Non-CV $=0.6$

\begin{tabular}{|c|c|c|c|c|c|c|c|c|}
\hline & \multicolumn{6}{|c|}{ CLMS $\left(\sigma_{\text {Non-CV }}=0.6\right)$} \\
\hline & & & \multicolumn{6}{|c|}{$\sigma_{\mathrm{CV}}$} \\
\hline & & & 0.5 & 0.6 & 0.7 & 0.8 & 0.9 & 1.0 \\
\hline \multirow{6}{*}{$\begin{array}{l}\text { CLMS } \\
\left(\sigma_{\text {Non-CV }}\right. \\
=0.6)\end{array}$} & \multirow{6}{*}{$\sigma_{\mathrm{CV}}$} & 0.5 & & & & & & \\
\hline & & 0.6 & 0.08 & & & & & \\
\hline & & 0.7 & 0.00 & 0.54 & & & & \\
\hline & & 0.8 & 0.00 & 0.01 & 0.00 & & & \\
\hline & & 0.9 & 0.00 & 0.00 & 0.00 & 0.06 & & \\
\hline & & 1.0 & 0.00 & 0.00 & 0.00 & 0.00 & 0.00 & \\
\hline
\end{tabular}

*Significant

There is no significant difference

The mean of sample in column is significantly higher than mean of sample in row

The mean of sample in row is significantly higher than mean of sample in column

When Non-CV compliance rate is $60 \%$ in the CLMS, throughput increases significantly with the growing of compliance rate in most cases. There is no significant difference between $50 \%$ and $60 \%, 60 \%$ and $70 \%$, and $80 \%$ and $90 \%$. 


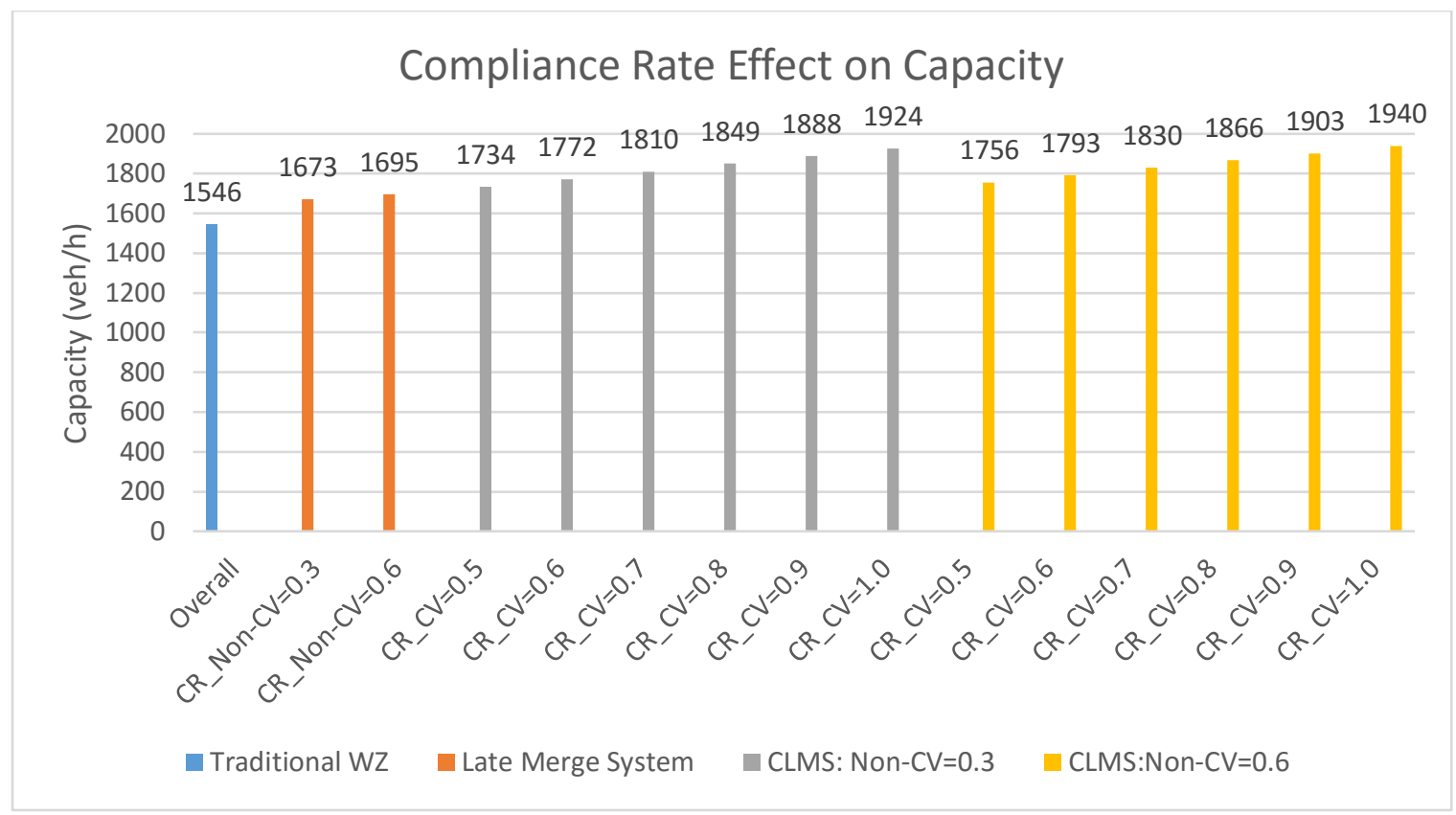

Figure 31 Compliance Rates Effect on Capacity in CLMS

Because capacity is represented by a single value in this experiment, the values in the graph can be compared. It seems that compliance rate in $\mathrm{CV}$ is more sensitive comparing to non-CV. Adding $10 \%$ of compliance rate in $\mathrm{CV}$ can make more evident difference than adding $30 \%$ in non-CV. 


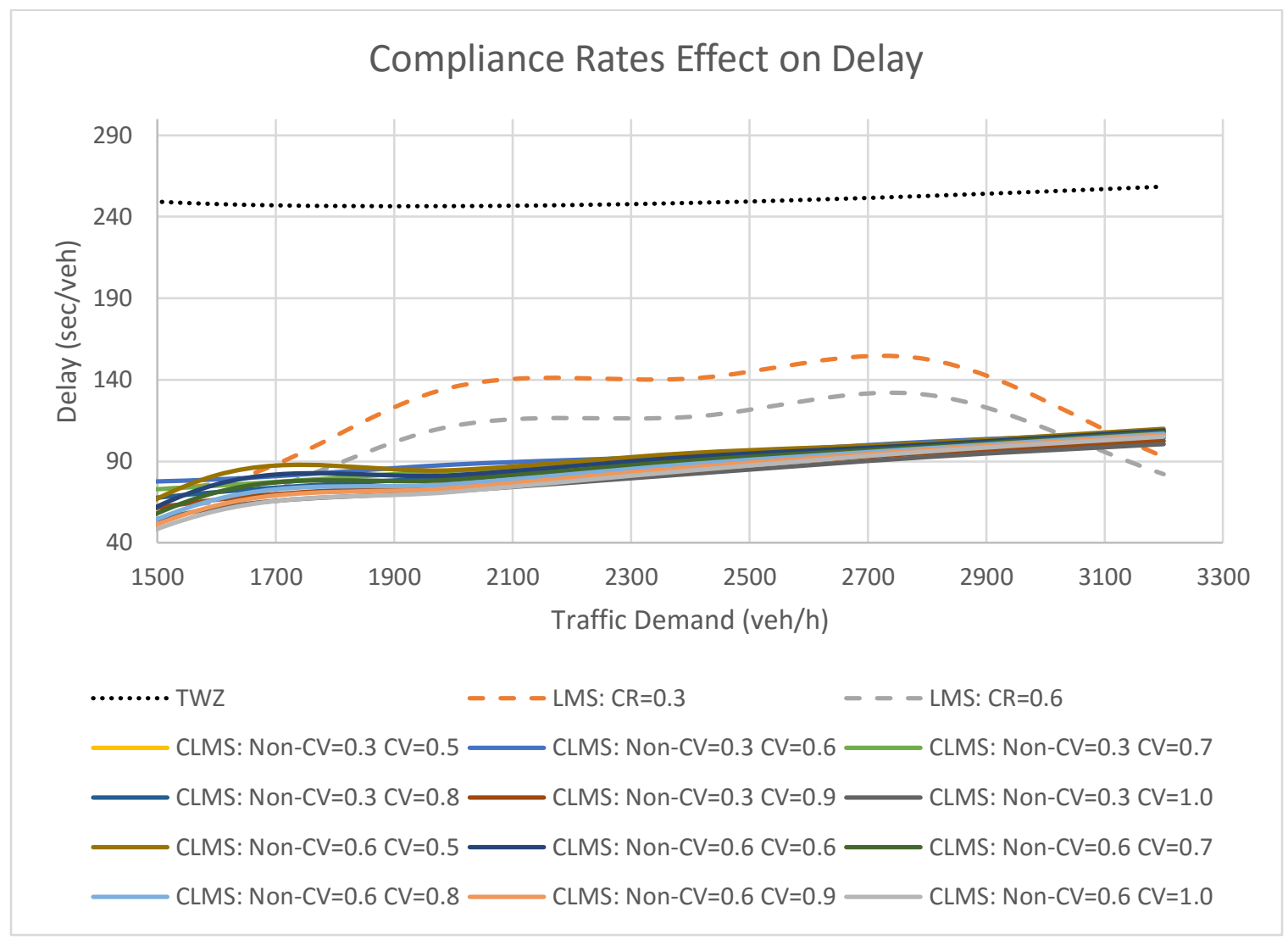

Figure 32 CV Compliance Rate Effect on Delay in CLMS

As figure 32 illustrates, late merge system has higher delay comparing with the CLMS. It can be noticed that there is a difference between the two compliance rates in late merge system. The difference between compliance rates in the CLMS seems minimal. Therefore, T-test is conduct to demonstrate the significance of difference between compliance rates. 
Table 44 T-Test of Compliance Rate Effect on Delay When $\sigma_{\text {Non-CV }}=0.3$

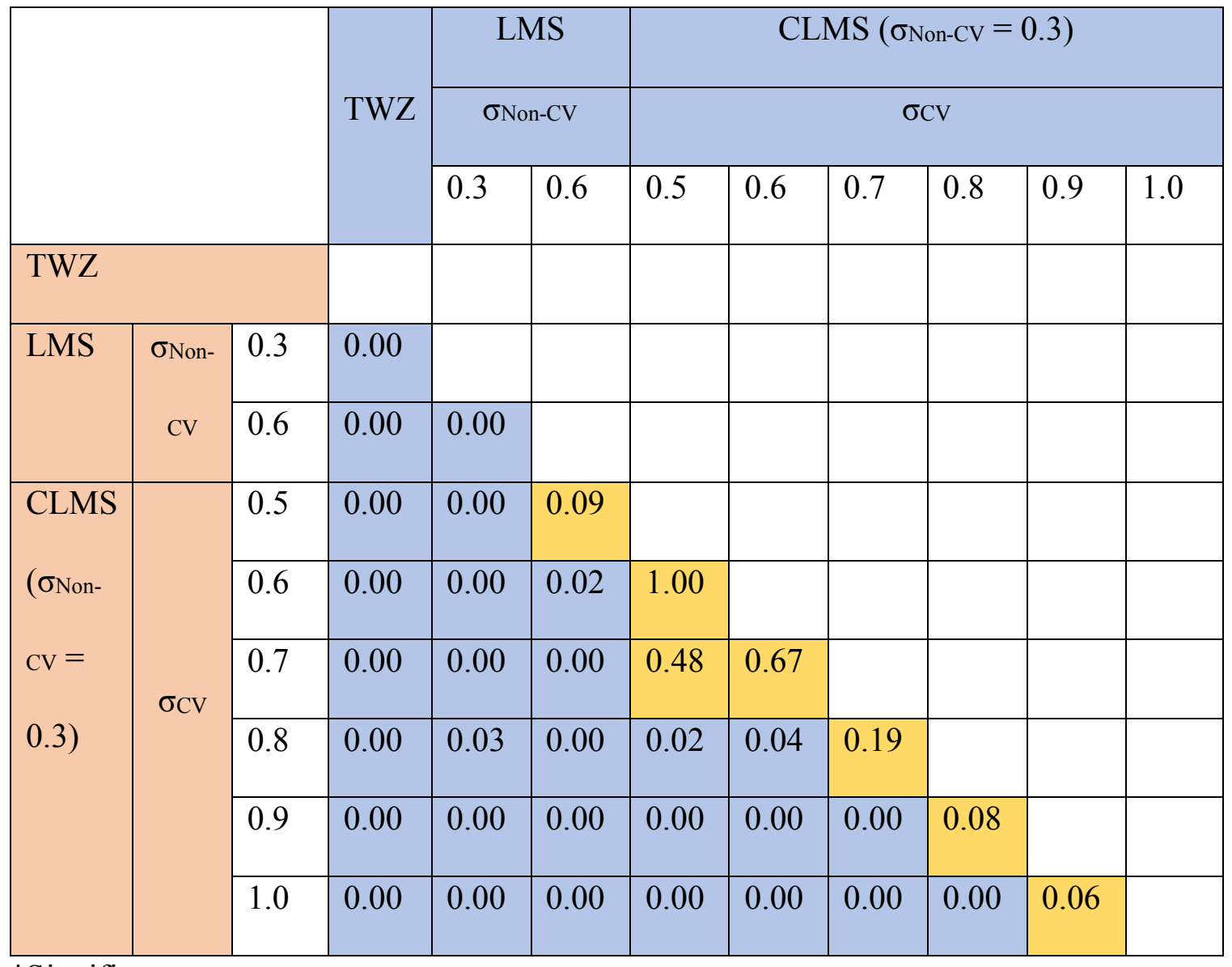

*Significant

There is no significant difference

The mean of sample in column is significantly higher than mean of sample in row

The mean of sample in row is significantly higher than mean of sample in column

From Table 45, it appears that there are significant differences between compliance rates in late merge and the CLMS. The difference of $10 \%$ in the CLMS, in terms of delay, is not significant as it requires $20 \%$ and more to show significant decrease. 
Table 45 T-Test of Compliance Rate Effect on Delay When $\sigma_{\text {Non-CV }}=0.6$

\begin{tabular}{|c|c|c|c|c|c|c|c|c|}
\hline & \multicolumn{6}{|c|}{ CLMS $\left(\sigma_{\text {Non-CV }}=0.6\right)$} \\
\hline & & & \multicolumn{6}{|c|}{$\sigma_{\mathrm{CV}}$} \\
\hline & & & 0.5 & 0.6 & 0.7 & 0.8 & 0.9 & 1.0 \\
\hline \multirow{6}{*}{$\begin{array}{l}\text { CLMS } \\
\left(\sigma_{\text {Non-CV }}\right. \\
=0.6)\end{array}$} & \multirow{6}{*}{$\sigma_{\mathrm{CV}}$} & 0.5 & & & & & & \\
\hline & & 0.6 & 0.32 & & & & & \\
\hline & & 0.7 & 0.05 & 0.20 & & & & \\
\hline & & 0.8 & 0.00 & 0.03 & 0.09 & & & \\
\hline & & 0.9 & 0.00 & 0.00 & 0.01 & 0.52 & & \\
\hline & & 1.0 & 0.00 & 0.00 & 0.00 & 0.00 & 0.41 & \\
\hline
\end{tabular}

*Significant

There is no significant difference

The mean of sample in column is significantly higher than mean of sample in row

The mean of sample in row is significantly higher than mean of sample in column

From the table above, the CLMS, when Non-CV compliance rate is $60 \%$, has lower delay when compliance rate of CV is higher. The difference of $10 \%$ in the CLMS is not significant as it requires $20 \%$ and more to show significant decrease. 


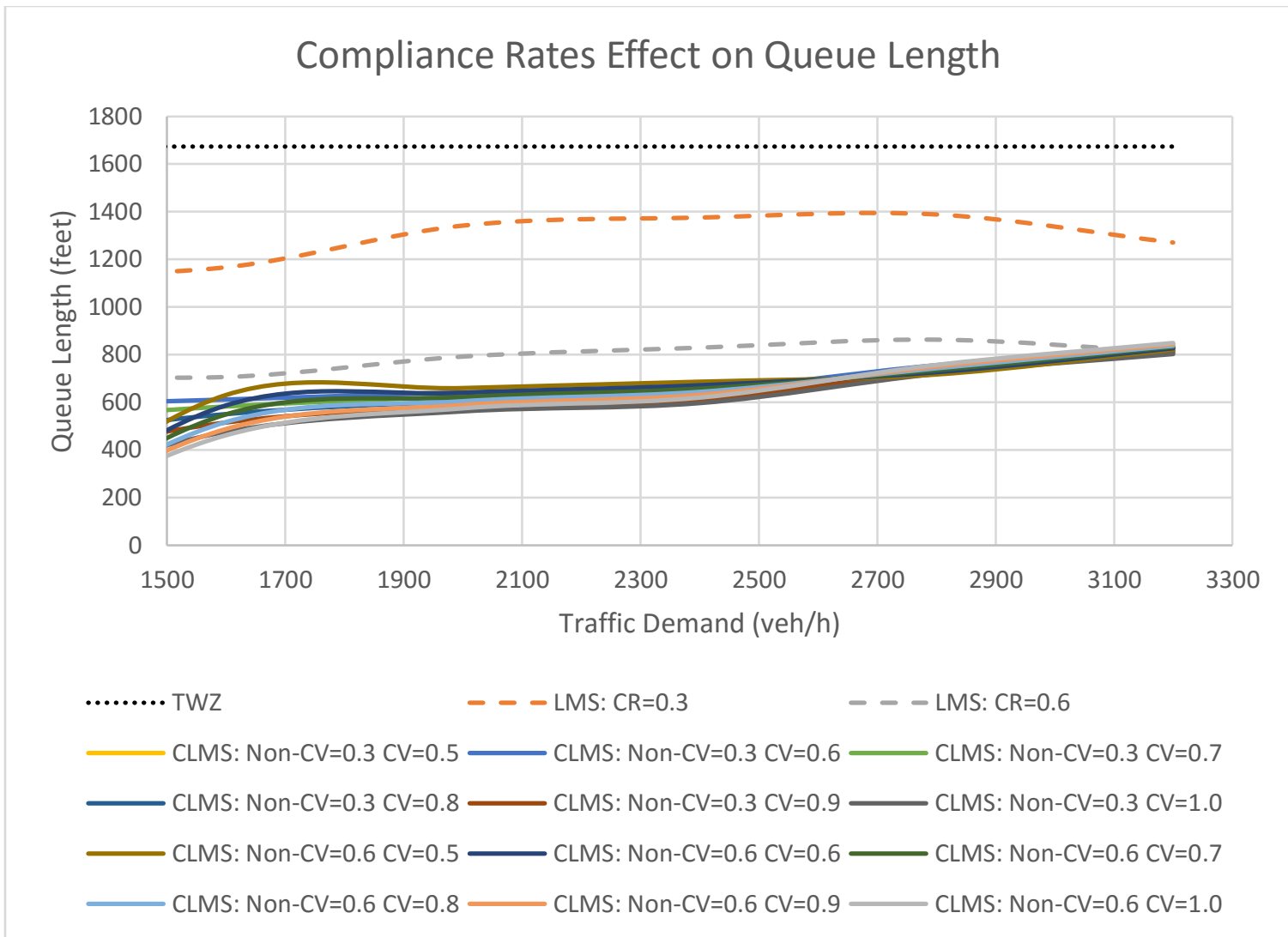

Figure 33 Non-CV Compliance Rate Effect on Queue Length in Late Merge System

As figure 33 shows, late merge system has higher delay comparing to the CLMS. It can be noticed that there is a difference between the two compliance rates in late merge system. The difference between compliance rates in the CLMS seems minimal. Therefore, T-test is conduct to demonstrate the significance of difference between compliance rates. 
Table 46 T-Test of Compliance Rate Effect on Queue Length When $\sigma_{\text {Non- } \mathrm{CV}}=0.3$

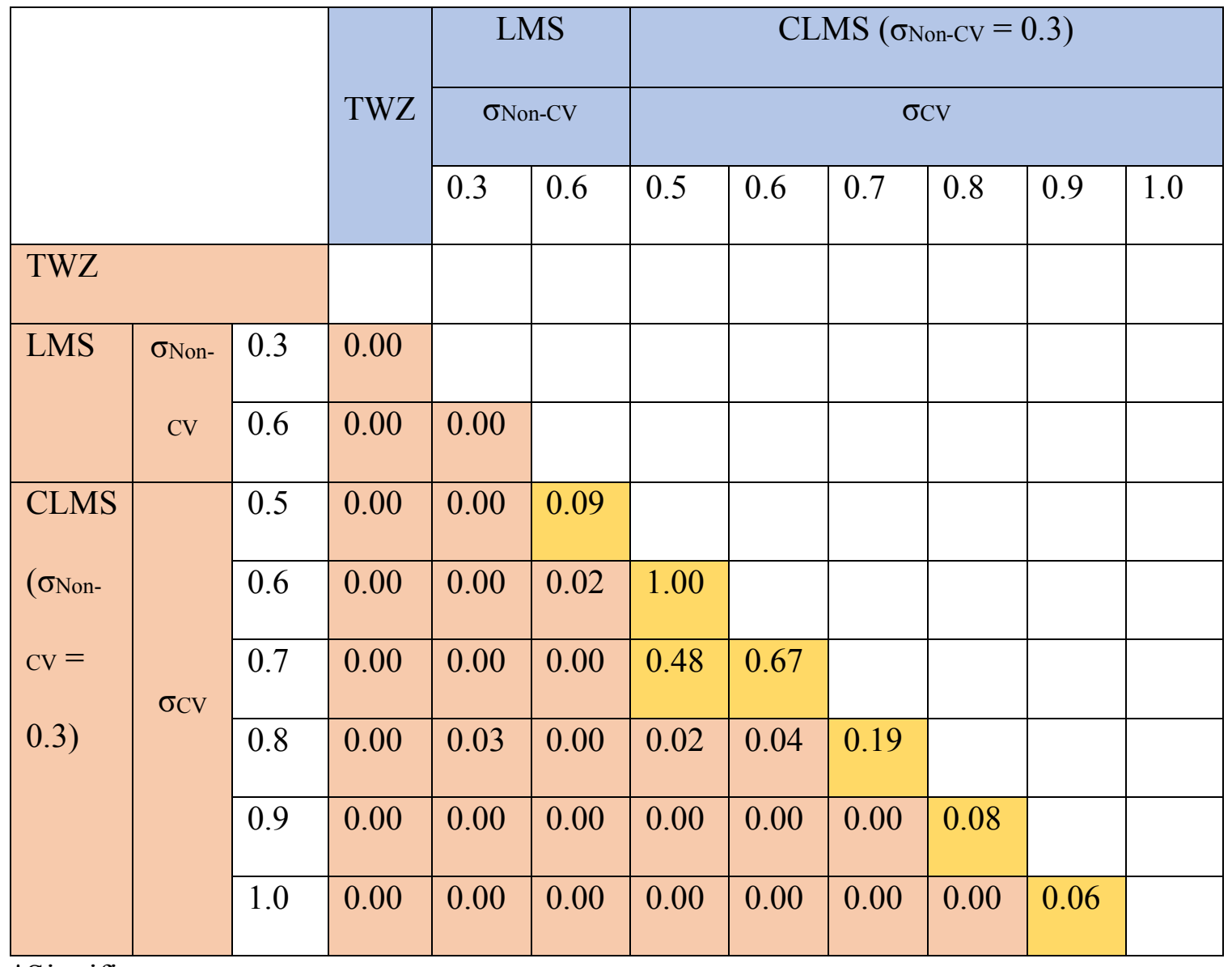

*Significant

\begin{tabular}{|l|l|}
\hline There is no significant difference & \\
\hline The mean of sample in column is significantly higher than mean of sample in row & \\
\hline The mean of sample in row is significantly higher than mean of sample in column & \\
\hline
\end{tabular}

From Table 45, it appears that there are significant differences between compliance rates in late merge and the CLMS. The difference of $10 \%$ in the CLMS, in terms of queue length, is not significant as it requires $20 \%$ and more to show significant decrease. 
Table 47 T-Test of Compliance Rate Effect on Queue Length When $\sigma$ Non-CV $=0.6$

\begin{tabular}{|c|c|c|c|c|c|c|c|c|}
\hline & \multicolumn{6}{|c|}{ CLMS $\left(\sigma_{\text {Non-CV }}=0.6\right)$} \\
\hline & & & \multicolumn{6}{|c|}{$\sigma_{\mathrm{CV}}$} \\
\hline & & & 0.5 & 0.6 & 0.7 & 0.8 & 0.9 & 1.0 \\
\hline \multirow{6}{*}{$\begin{array}{l}\text { CLMS } \\
\left(\sigma_{\text {Non-CV }}\right. \\
=0.6)\end{array}$} & \multirow{6}{*}{$\sigma_{\mathrm{CV}}$} & 0.5 & & & & & & \\
\hline & & 0.6 & 0.32 & & & & & \\
\hline & & 0.7 & 0.05 & 0.20 & & & & \\
\hline & & 0.8 & 0.00 & 0.03 & 0.09 & & & \\
\hline & & 0.9 & 0.00 & 0.00 & 0.01 & 0.52 & & \\
\hline & & 1.0 & 0.00 & 0.00 & 0.00 & 0.00 & 0.41 & \\
\hline
\end{tabular}

*Significant

There is no significant difference

The mean of sample in column is significantly higher than mean of sample in row

The mean of sample in row is significantly higher than mean of sample in column

From the table above, the CLMS, when Non-CV compliance rate is $60 \%$, has lower delay when compliance rate of CV is higher. The difference of $10 \%$ in the CLMS is not significant as it requires $20 \%$ and more to show significant decrease.

\subsection{Evaluation of CLMS}

From the analysis above, the CLMS has shown an improvement of the traffic operation in terms of throughput, capacity, demand at $0.9 \mathrm{~V} / \mathrm{C}$, delay and queue length. There is a significant gap between the CLMS and the traditional work zone system in moderate and high traffic demands. Therefore, most of comparisons were between CLMS and late 
merge system. The analysis demonstrated that the CLMS, as any other late merge system, operates well during moderate traffic demand. Additionally, the system has proved an improved operations in some MOEs at high traffic demands.

The improved performance of CLMS is dependent on the measure of effectiveness and other variables such as traffic demand, lane distribution, compliance rate and market penetration rate. Therefore, the following section includes a selection guide for the CLMS and other system according to MOEs and specific variables. 


\section{CHAPTER V}

\section{DISCUSSIONS AND CONCLUSIONS}

As the results showed that the CLMS improved the performance in most of MOEs, this section discusses and concludes the experiment findings.

\subsection{Discussions}

There are different MOEs that can be inspected to decide the feasibility of a system. Therefore, this study considered several MOEs including throughput, capacity, V/C, delay and queue length. The three compared systems showed different performance against each MOE. First of all, traditional work zone has never performed better than any other system and therefore, most of comparisons were limited between late merge system and the CLMS. The simulation results revealed an overall improved performance of the CLMS compared to traditional work zone system and late merge system. At the low traffic demand where volume is significantly lower than capacity, all systems can demonstrate efficient traffic operations. At the same time, the performance at high traffic demand is relatively similar between late merge system and the CLMS. At moderate traffic demand, the CLMS has demonstrated an improved performance. 
Throughput results at lower traffic demand are the same due to the high capacity-tovolume ratio at such low traffic. Thus, using traditional work zone system would be more feasible because of its lower cost and its easy operation plan. When traffic demand is moderate, using CLMS would result in better throughput. The results of traffic demand suggest using late merge system although the difference is not substantially higher. To improve capacity-to-volume ratio (V/C), the CLMS with a market penetration rate of $40 \%$ or higher can do the job. Again, late merge system performed efficiently at higher traffic demand when $\mathrm{V} / \mathrm{C}$ is the major MOEs.

Delay results proved that late merge system can be efficient at both lower and higher traffic demand. The CLMS performed well at moderate traffic demand and it was sensitive to the late merge area depending on the reduction factor. When reduction factor is 0.2 , the CLMS should have a late merge area of 200 feet, while in the case of reduction factor of 0.4 , a late merge area of 500 feet can perform efficiently. Traditional work zone system showed significantly high queue length over all traffic demand. The main reason is because of early merge behavior, the traffic backup in the open lane reached the maximum length of simulation network. At all traffic demands, the CLMS appeared with an improved performance. It is suggested using late merge area of 200 feet when reduction factor is 0.2 , while in the case of reduction factor of 0.4 , a late merge area of 500 feet is optimal.

As mentioned earlier, the CLMS has the operation plan to activate the system at certain traffic demand. Therefore, activating CLMS at the traffic demand threshold would improve operations performance of work zones. 


\subsubsection{Selection Guidelines}

By examining the three work zone systems with different MOEs, the CLMS has shown improved operations. Indeed, compliance rates and market penetration rates are significant variables that would enhance the CLMS. Also, traffic characteristics such as heavy vehicle percentage and lane distribution play significant roles in finding the value of MOEs. A general selection guide was developed based on the analysis to determine the appropriate system (see the following table).

Table 48 Selection Guide for Work Zone Control Strategy

\begin{tabular}{|l|c|c|c|c|c|c|c|}
\hline \multicolumn{2}{|c|}{} & \multicolumn{7}{|c|}{ Traffic Demand (Veh/h) } \\
\cline { 3 - 8 } \multicolumn{2}{|c|}{} & 1200 & 1600 & 2000 & 2400 & 2800 & 3200 \\
\hline \multicolumn{2}{|l|}{ Throughput } & & & & & & \\
\hline \multirow{2}{*}{ Capacity } & $F=0.2$ & $\psi=200$ & $\psi=200$ & $\psi=200$ & $\psi=200$ & $\psi=200$ & $\psi=200$ \\
\cline { 2 - 8 } & $F=0.4$ & $\psi=1000$ & $\psi=1000$ & $\psi=1000$ & $\psi=1000$ & $\psi=1000$ & $\psi=1000$ \\
\hline 0.9 V/C & & $\omega \geq 40 \%$ & $\omega \geq 40 \%$ & $\omega \geq 40 \%$ & & \\
\hline \multirow{2}{*}{ Delay } & $F=0.2$ & & & $\psi=200$ & $\psi=200$ & $\psi=200$ & \\
\cline { 2 - 8 } & $F=0.4$ & & & $\psi=500$ & $\psi=500$ & $\psi=500$ & \\
\hline \multirow{2}{*}{$\begin{array}{l}\text { Queue } \\
\text { Length }\end{array}$} & $F=0.2$ & $\psi=200$ & $\psi=200$ & $\psi=200$ & $\psi=200$ & $\psi=200$ & $\psi=200$ \\
\cline { 2 - 8 } & $F=0.4$ & $\psi=500$ & $\psi=500$ & $\psi=500$ & $\psi=500$ & $\psi=500$ & $\psi=500$ \\
\hline
\end{tabular}

\begin{tabular}{|l|l|}
\hline Traditional Work Zone \\
\hline Late Merge System & \\
\hline CLMS & \\
\hline
\end{tabular}

\begin{tabular}{|l|l|}
\hline$\psi$ & Late Merge Area \\
\hline$F$ & Reduction Factor for Gap Acceptance \\
\hline$\omega$ & Market Penetration Rate \\
\hline
\end{tabular}




\subsection{Conclusions and Future Research}

In conclusion, work zone traffic operations can be improved by applying different control strategy. The development of late merge strategy was not enough to substantially improve the situation due to the lack of compliance and communication among drivers. The CLMS principle is to enhance the opportunity of cooperative late merge via the new technology, connected vehicles (CV). The main principle is based on improving the lack of communication and encourage drivers to cooperate in merging process. By developing the system and designing the layout, different scenarios of interactions between $\mathrm{CV}$ and non-CV were considered. These scenarios facilitated planning the main logic of the CLMS and drawing flowcharts of the algorithm. Microscopic car-following and lanechanging models were selected and updated to fit with the main goal of the system and purpose of this study. The study of recognized lane-changing microscopic models led to choosing Hidas (2005) because it considers the cooperation effect on lane-changing behavior. To reflect possible scenarios, lane-changing model was updated and programmed to operate on the simulation software package (VISSIM). In this study, carfollowing model (Wiedemann 99) provided by VISSIM was utilized as it fits the purpose of simulation.

Simulation experiment was designed to include a traditional work zone system and a late merge system in order to compare them with the performance of CLMS. The experiment design considered eight different variables with multiple levels. These variables include traffic characteristics, and design and deriving behavior parameters. Testing all variables different levels of traffic demand, heavy vehicle percentage, lane distribution, late merge distance, compliance of $\mathrm{CV}$ and non-CV, reduction factor of gap acceptance and market 
penetration rate created 2070 simulation scenarios. To ensure the statistical significance of the results, 30 runs for each simulation scenario were conducted, which resulted a total of 62,100 simulation runs.

Simulation output included major measures of effectiveness including throughput, capacity, V/C ratio, delay and queue length. The data was interpreted using statistical tests and graphs to analyze the performance of the three systems. The analysis results revealed the significant variables that impact each system and found multiple regression models for each of them.

The CLMS demonstrated an improved traffic operation performance at most of traffic demands in all considered MOEs, especially, in the case of moderate traffic demand. The system is capable to increase throughput and capacity of work zone as well as to decrease the delay and queue length at most of traffic conditions. From the system performance, the following points can be concluded:

- The CLMS provided significantly improved throughput, especially, at traffic demands between 1600 and $2800 \mathrm{veh} / \mathrm{h}$;

- When reduction factor was 0.2 , the capacity of the CLMS was at its highest when late merge area was 200 feet;

- When reduction factor was 0.4 , the capacity of the CLMS was at its highest when late merge area was 1000 feet;

- The traffic demand when $\mathrm{V} / \mathrm{C}=0.9$ could be significantly increased when market penetration rate was $40 \%$ and higher;

- Delay was significantly lower at the CLMS at traffic demands between 2000 and $2800 \mathrm{veh} / \mathrm{h}$ when reduction factor was 0.2 and late merge area was 200 feet; 
- Delay was significantly lower at the CLMS ta traffic demands between 2000 and $2800 \mathrm{veh} / \mathrm{h}$ when reduction factor was 0.4 and late merge area was 500 feet;

- At all traffic demands, queue length was lower when reduction factor was 0.2 and late merge area was 200 feet;

- At all traffic demands, queue length was lower when reduction factor was 0.2 and late merge area was 500 feet

- The CLMS is impacted by the compliance rate of both CV and non-CV

- There is a significant difference between $30 \%$ and $60 \%$ compliance rate of non$\mathrm{CV}$ in the CLMS in all MOEs;

- There is significant differences between compliance rates of CV in all MOEs, except in some cases when $10 \%$ increase did not show a sign of significance.

In this study, the change of gap acceptance by CV drivers were assumed due to the lack of research about the effect of $\mathrm{CV}$ on gap acceptance. To achieve more accurate results about the performance of CLMS in particular and using other CV application in general, Future studies should consider conducting a study about the gap acceptance behavior by $\mathrm{CV}$ drivers. Also, the level of compliance rate for both $\mathrm{CV}$ and non-CV should be measured in future studies. Another study can investigate the performance of CLMS Level 2 and CLMS Level 3 as well. 


\section{REFERENCES}

Al-Kaisy, A., M. Zhou, and F. Hall. "New Insights into Freeway Capacity at Work Zones Empirical Case Study." Transportation Research Record 1710 (TRB, National Research Council), 2000: 154-160.

Dudek, C. L., and S. H. Richards. "Traffic Capacity Through Urban Freeway Work Zones in Texas." Transportation Research Record 869 (TRB, National Research Council), 1982: 14-18.

Transportation Research Board. Highway Capacity Manual 2000. Washington, D.C., 2000.

Dixon, K. K., J. E. Hummer, and A. R. Lorscheider. "Capacity for North Carolina Freeway Work Zones." Transportation Research Record 1529 (TRB, National Research Council), 1996: $27-34$.

Nemeth, Z.A. and N.M. Roupahil. Lane Closures at Freeway Work Zones: Simulation Study. Journal of the Transportation Research Record. No. 869. 1982. pp. 19-25.

Mousa, R.M., N.M. Rouphail, and F. Azadivar. Integrating Microscopic Simulation and Optimization: Application to Freeway Work Zone Traffic Control. Journal of the Transportation Research Record. No. 1254. 1990.

Tarko, Andrzej and Venugopal, Shyam. 2001. Safety and Capacity Evaluation of the Indiana Lane Merge System. West Lafayette : INDOT, 2001. FHWA/IN/JTRP-2000/19. 
Pesti, G., D.R. Jessen, P.S. Byrd and P.T. McCoy, "Traffic Flow Characteristics of the Late Merge Work Zone Control Strategy.” In Transportation Research Record 1657, TRB National Research Council, Washington D.C., 1999, pp. 1-9.

McCoy, P.T. and Pesti, G. "Dynamic Late Merge Control Concept for Work Zones on Rural Interstate Highways,” Presented at the TRB 80th Annual Meeting, 2001.

Beacher, Andrew G., Fontaine, Michael D. and Garber, Nicholas J. 2005. Guidelines for Using Late Merge Traffic Control in Work Zones: Results of a Simulation Based Study. Transportation Research Record: Journal of the Transportation Research Board. 2005.

URS, “Dynamic Late Merge System Evaluation: Initial Deployment on US-10," Minnesota Department of Transportation, 2003.

Kang, K., G. Chang, and J. Paracha. Dynamic Late Merge Control at Highway Work Zones: Evaluation, Observations, and Suggestions. Transportation Research Record, No. 1948, TRB, National Research Council, Washington, D.C., 2006, pp. 86-95.

Grillo, L. F., Datta, T. K. and Hartner, C. 2008. Evaluation of the dynamic late lane merge system at freeway construction work zones. Transportation Research Record: Journal of Transportation Research Board, 2055: 3-10.

Radwan, Essam and Harb, Rami. 2009. Dynamic Early Merge and Dynamic Late Merge at Work Zone Lane Closures. The ITE Technical Conference and Exhibits held at Phoenix, Arizona. 2009.

Uhlemann, E. Introducing Connected Vehicles [Connected Vehicles], in IEEE Vehicular Technology Magazine, vol. 10, no. 1, pp. 23-31, March 2015. doi:

10.1109/MVT.2015.2390920

Chang, J., G. Hatcher, D. Hicks, J. Schneeberger, B. Staples, S. Sundarajan, M. Vasudevan, P. Wang, and K. Wunderlich. Estimated Benefits of Connected Vehicle Applications: Dynamic Mobility Applications. U.S. Department of Transportation ITS Joint Program Office, Washington, DC, 2015.

Maitipe, B. R. (2011). "Development and field demonstration of DSRCbased traffic information system for the work zone.” Ph.D. thesis, Univ. of Minnesota, Minneapolis 
Genders, W. and S. Razavi "Impact of connected vehicle on work zone network safety through dynamic route guidance," Journal of Computing in Civil Engineering, vol. 30, no. 2, Article ID 04015020, 2016

Davis, L. C. Improving traffic flow at a 2-to-1 lane reduction with wirelessly connected, adaptive cruise control vehicles. Physica A, 451 (2016), pp. 320-332

Abdulsattar, H., A. Mostafizi and H. Wang. 2017 "Assessing the Impacts of ConnectedVehicle Technology on Work Zone Rear-End Collisions: Agent-Based Modeling Approach". Transportation Research Record: Journal of Transportation Research Board HCM2000, Highway Capacity Manual 2000 by Transportation Research Board Gipps, P.G., 1986. A Model for the Structure of Lane-Changing Decisions. Transport. Res. 20B, 107-120.

Fritzsche, H.-T., 1994. A model for traffic simulation. Traffic Eng. Control 35 (5), $317-$ 321.

Yang, Q. and H. N. Koutsopoulos (1996). A microscopic traffic simulator for evaluation of dynamic traffic management systems. Transportation Research C 4(3), 113-129.

Ahmed, K. I. 1999. Modeling Drivers' Acceleration and Lane Changing Behavior. Department of Civil and Environmental Engineering, Massachusetts Institute of Technology, Master of Science.

Kita, H. (1999). A merging-giveway interaction model of cars in a merging section: a game theoretic analysis. Transportation Research Part A,33(3), 305-312. doi:10.1016/S0965-8564(98)00039-1

Hidas, P. (2005). Modelling vehicle interactions in microscopic simulation of merging and weaving. Transportation Research Part C, 13(1), 37-62.

doi:10.1016/j.trc.2004.12.003 
Chowdhury, D., Wolf, D. E., \& Schreckenberg, M. (1997). Particle hopping models for two-lane traffic with two kinds of vehicles: Effects of lane-changing rules. Physica A: Statistical Mechanics And Its Applications, 235(3-4), 417-439. doi:10.1016/S03784371(96)00314-7

Nassab, K., Schreckenberg, M., Ouaskit, S., \& Boulmakoul, A. (2005). Impacts of different types of ramps on the traffic flow. Physica A, 352(2-4).

Gipps, P. G. (1986). "A model for the structure of lane-changing decisions " Transportation Research Part B: Methodological 20(5): 403-414.

Toledo, T. 2003. Integrated Driving Behavior Modeling. Department of Civil and Environmental Engineering, Massachusetts Institute of Technology, Doctor of Philosophy.

Elefteriadou, Lily. 2014. An Introduction to Traffic Flow Theory. s.1. : Springer, 2014.

Virginia Department of Transportation. 2013. Traffic Operations Analysis Tool Guidebook. s.1. : VDOT Traffic Engineering Division, 2013.

Talebpour, H. S. Mahmassani, S. H. Hamdar, "Modeling lane-changing behavior in a connected environment: A game theory approach", Transp. Res. C Emerg. Technol., vol. 59, pp. 216-232, Oct. 2015.

Manual on Uniform Traffic Control Devices (MUTCD). FHWA, U.S. Department of Transportation, Washington, DC, 2009

Liu, H., Wei, H., Zuo, T., Li, Z., \& Yang, Y. J. (2017). Fine-tuning ADAS algorithm parameters for optimizing traffic safety and mobility in connected vehicle environment. Transportation Research Part C: Emerging Technologies, 76, 132-149.

Krammes, R. A., \& Lopez, G. O. (1992). Updated short-term freeway work zone lane closure capacity values.

Ullman, G. L., Dudek, C. L., \& Ullman, B. R. (2005). Development of a field guide for portable changeable message sign use in work zones (No. FHWA/TX-06/0-4748-2). Texas Transportation Institute, Texas A \& M University System. Chicago 
Nobukawa, K., Bao, S., LeBlanc, D. J., Zhao, D., Peng, H., \& Pan, C. S. (2016). Gap Acceptance During Lane Changes by Large-Truck Drivers-An Image-Based Analysis. IEEE transactions on intelligent transportation systems, 17(3), 772-781.

Systematics, C. (2009). Performance measurement framework for highway capacity decision making. Strategic Highway Research Program Report. 


\section{APPENDIX}

\section{T-test Results from Minitab}

\section{Example from Throughput:}

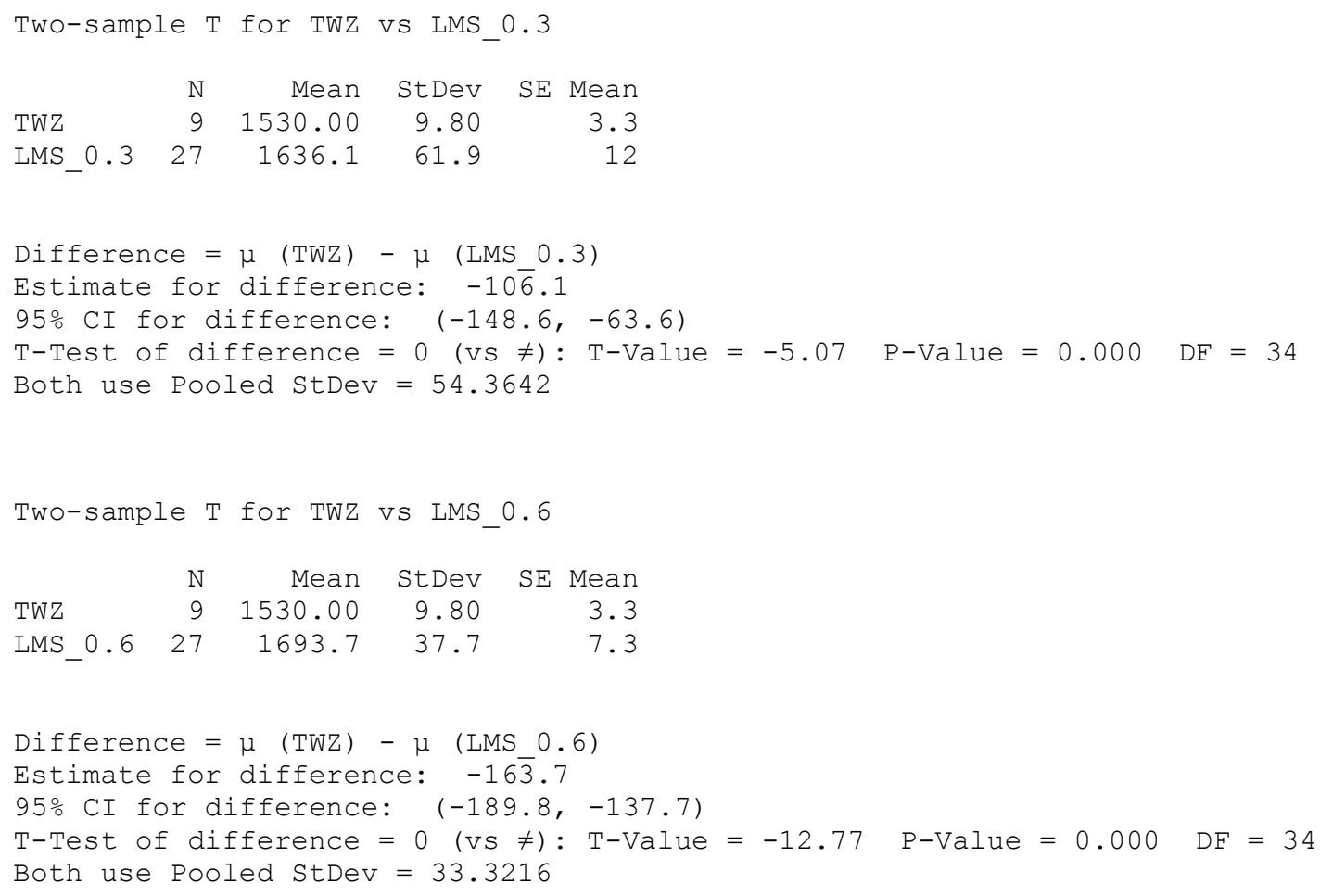




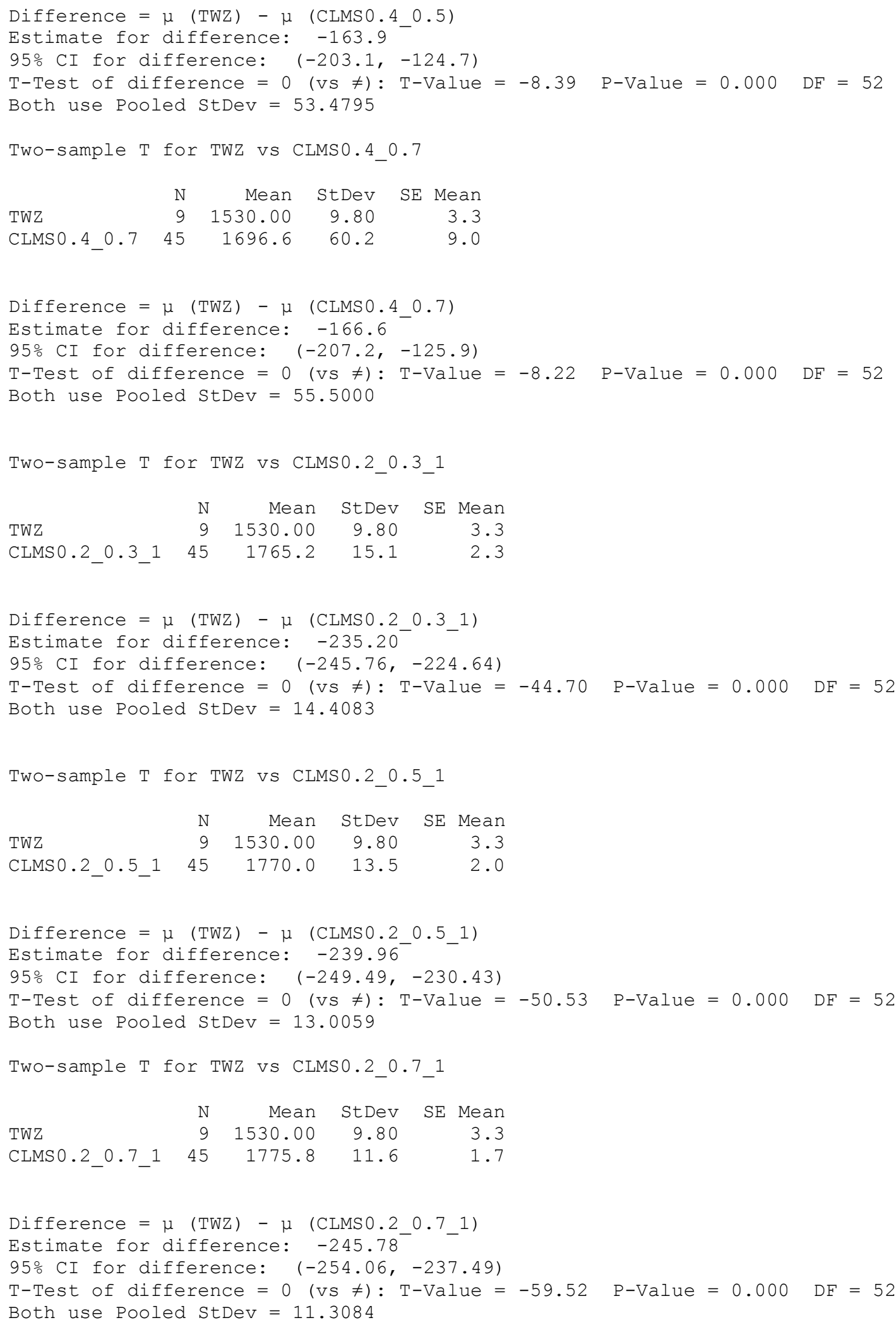




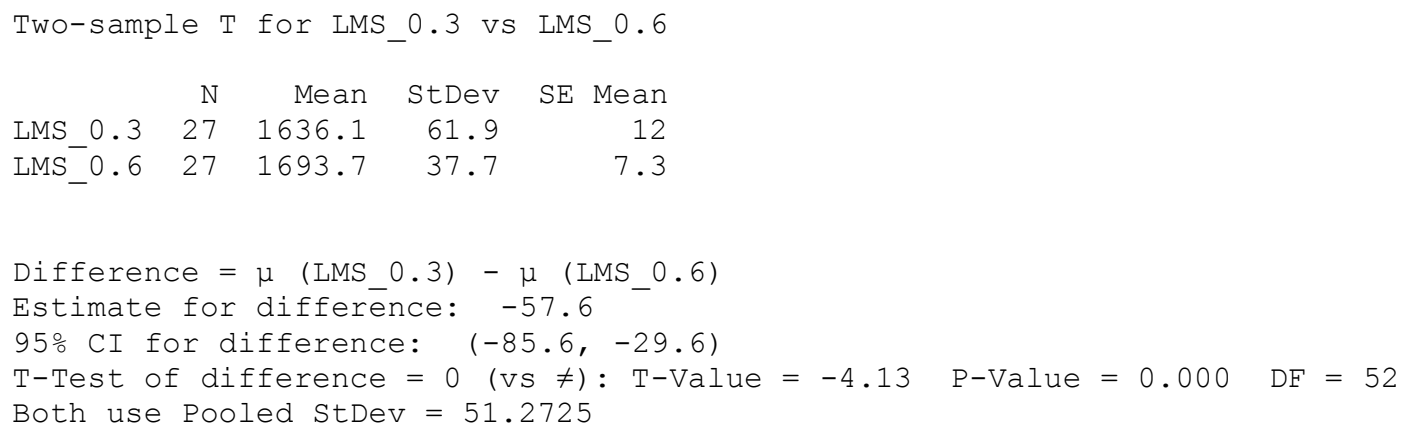




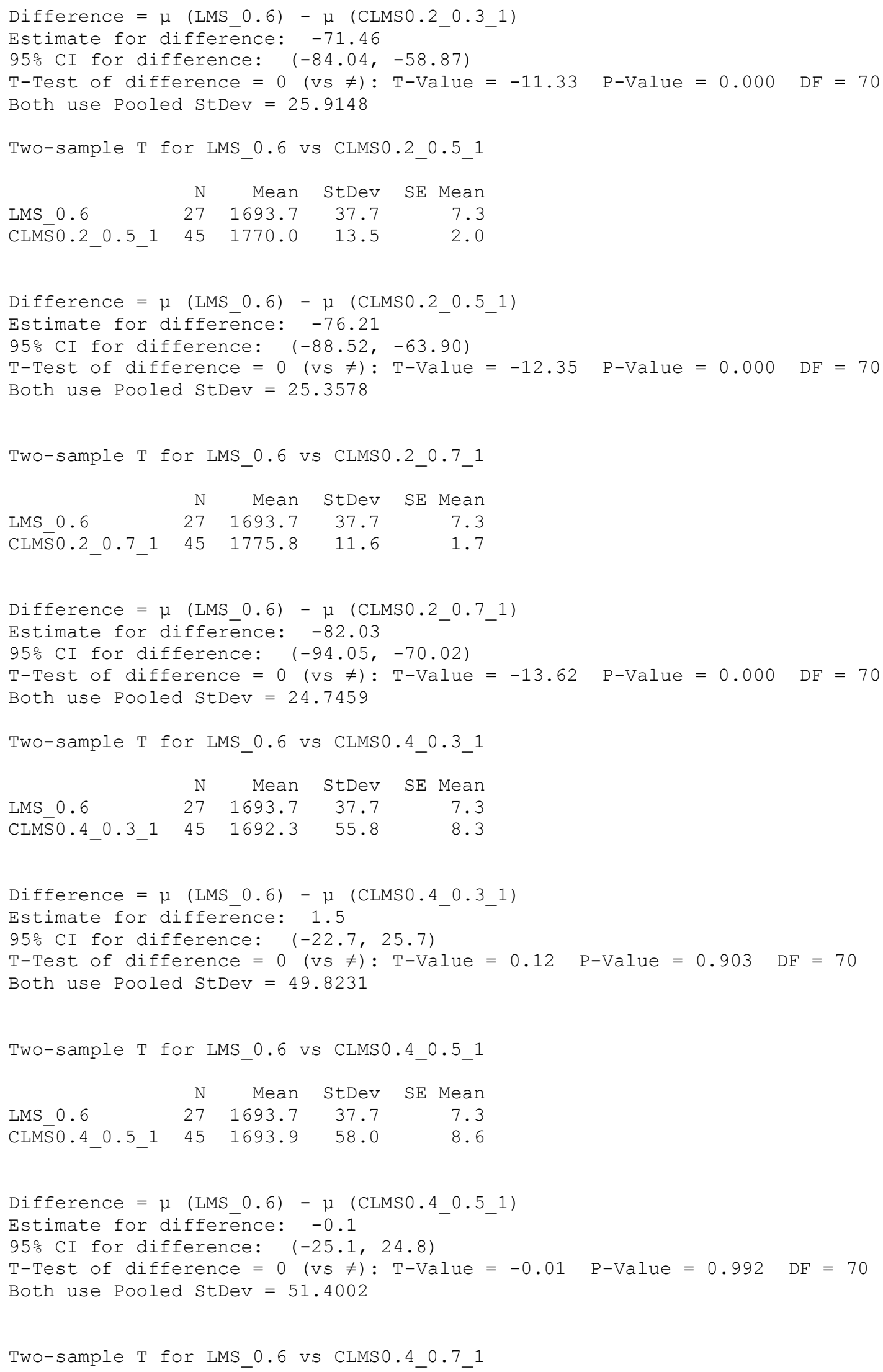




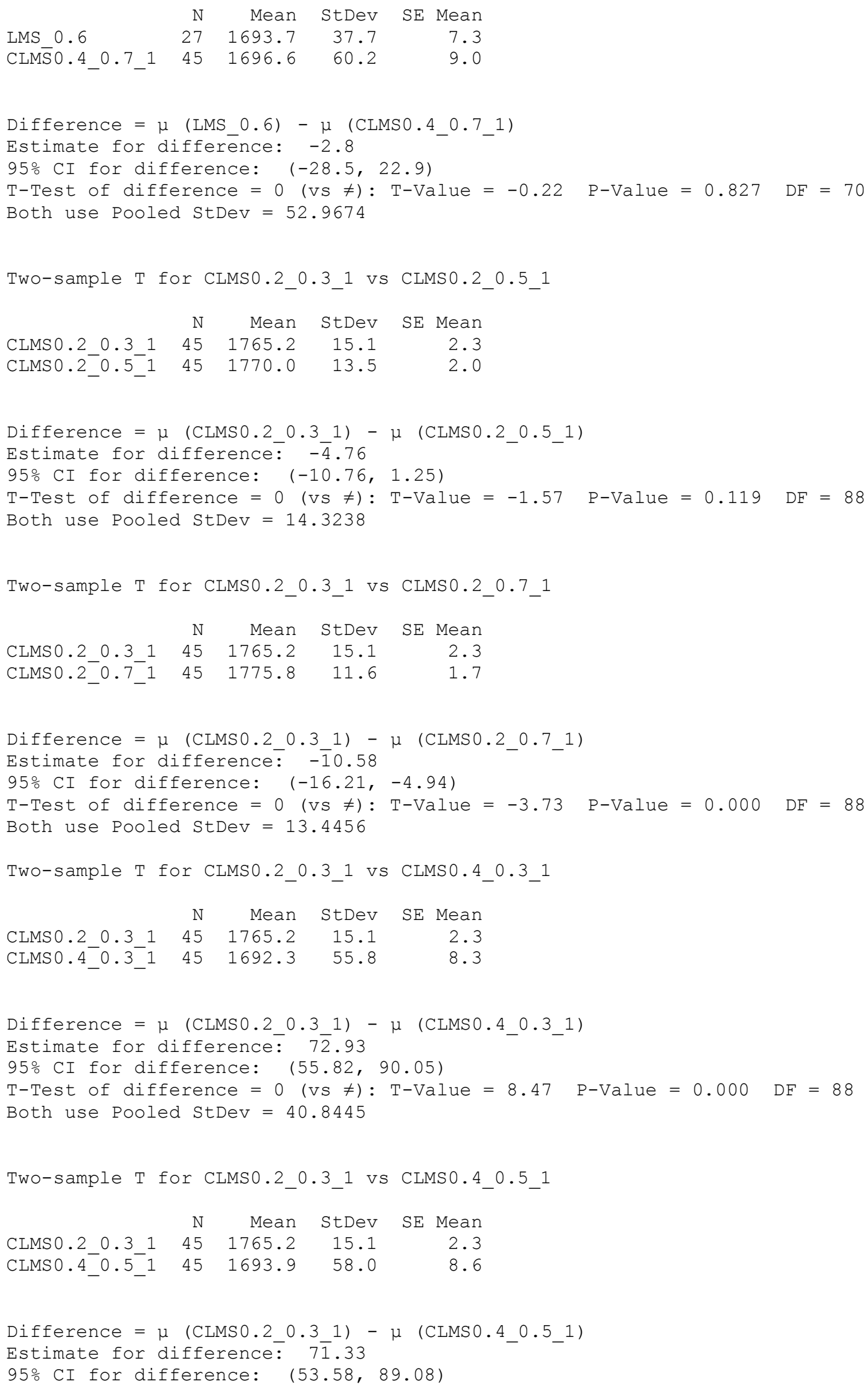




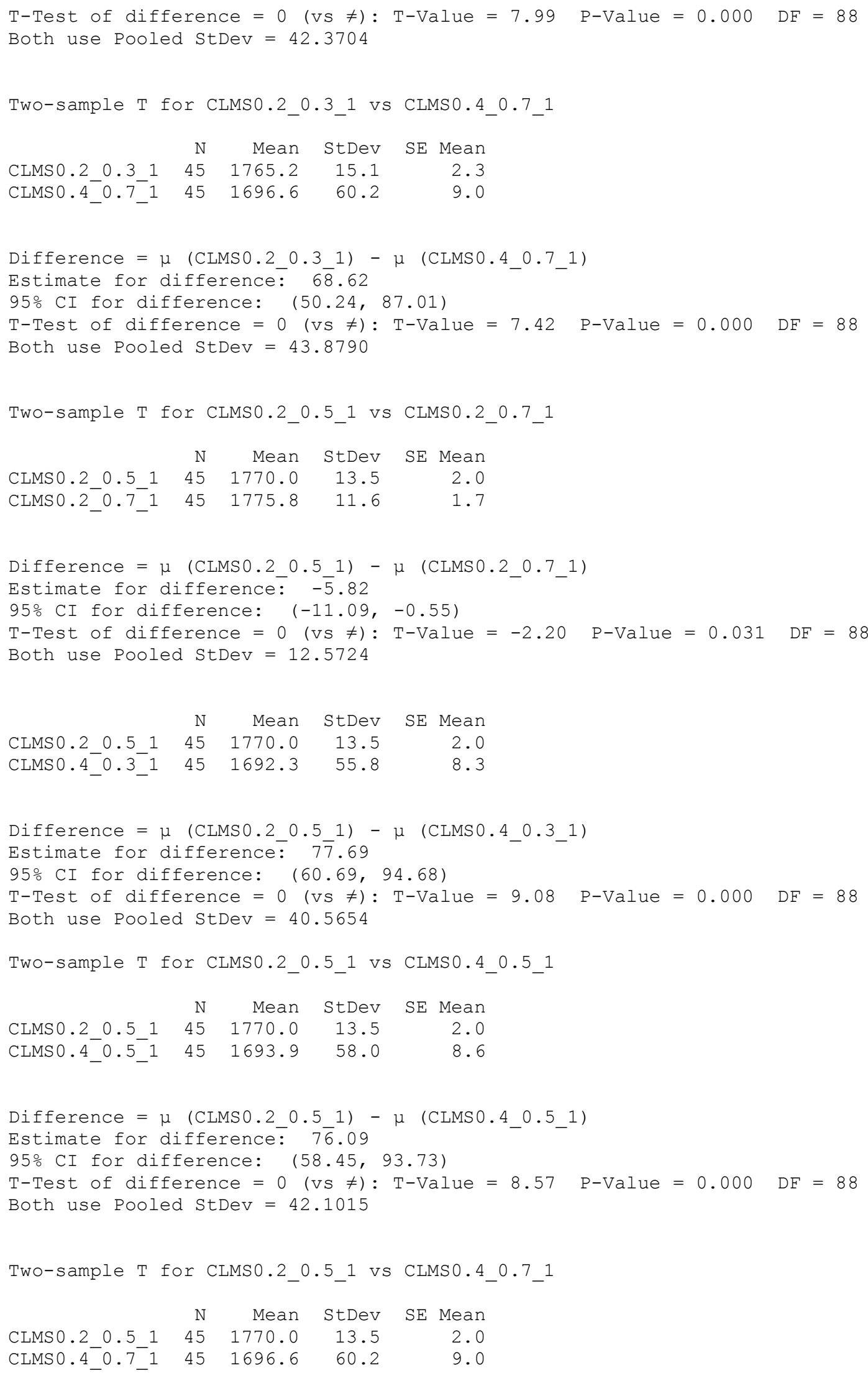




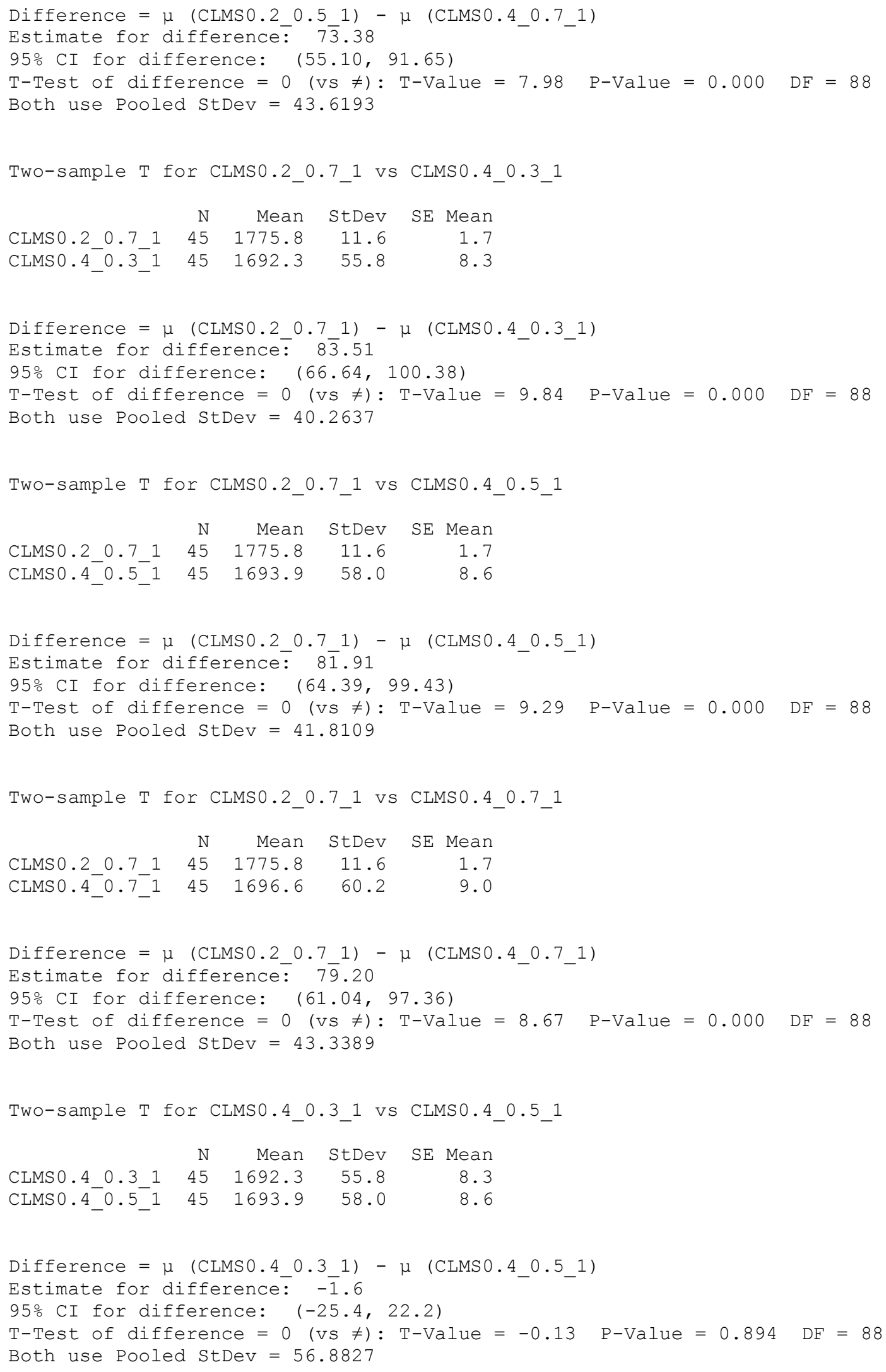




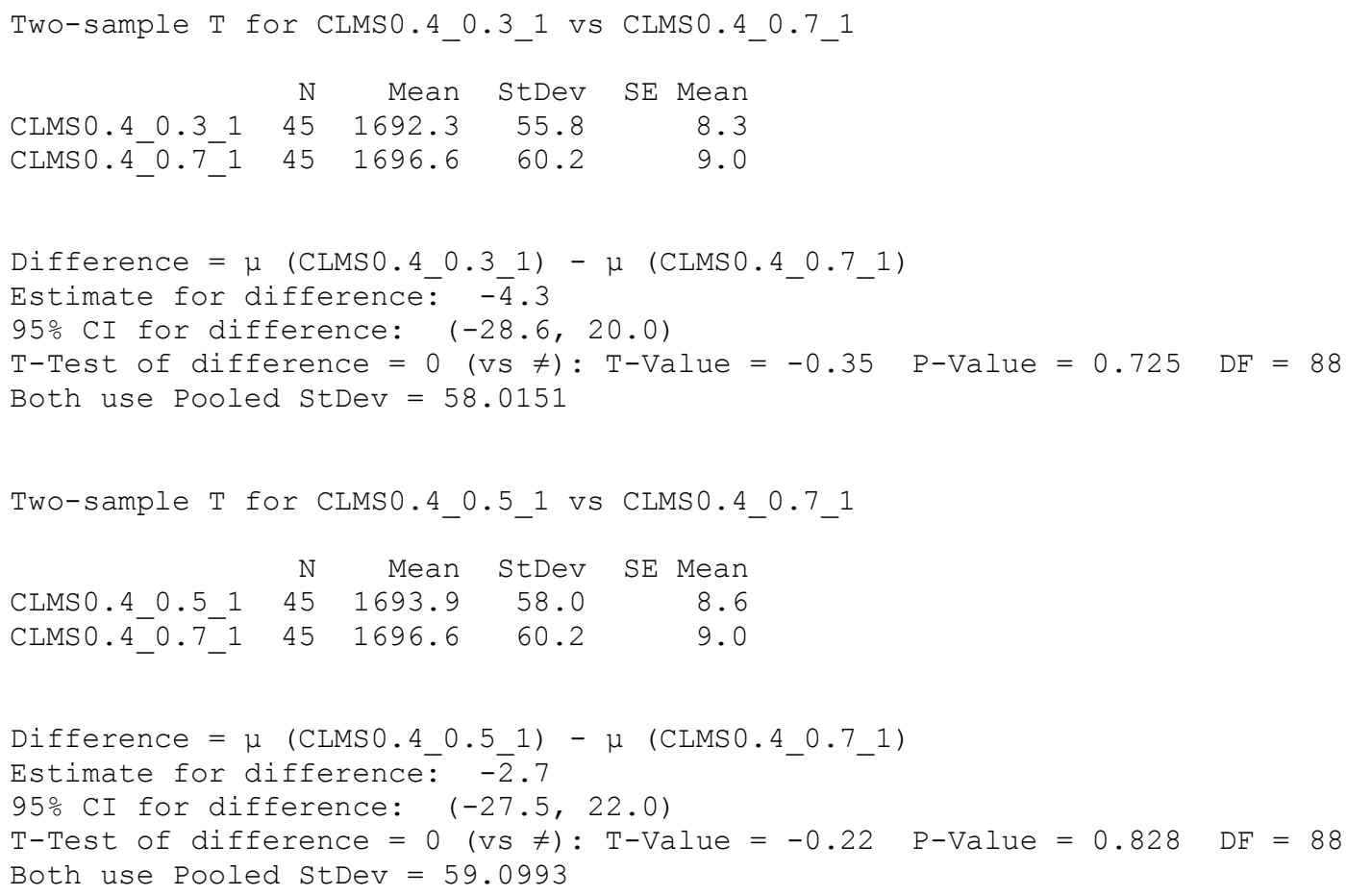




\title{
CURRICULUM VITA
}

\author{
Abdulmaged Algomaiah
}

Contact Details

amalgo01@louisville.edu

(502) 389-3563
Home Address $330 \mathrm{~W}$ Bloom St

Louisville, KY 40208

\section{EDUCATION}

Master of Science, Civil Engineering (Transportation)

University of Louisville, Louisville, KY

May 2015 - August 2017

THESIS - A Highway Smart Work Zone Merge System Enabled by Connected Vehicles

Bachelor of Science, Civil Engineering

Second Major, Transportation and Planning

Queensland University of Technology, Brisbane, QLD

Feb 2010 - Dec 2014

THESIS - Modelling Bus-Bunching Phenomenon from Empirical Data

\section{TRAINING}

Executive Decision Making Workshop

University of Melbourne, VIC, Australia

Leadership Development and Team Building

University of Queensland, QLD, Australia

Sustainability in Engineering

University of Kassel, Hessen, Germany 


\section{AWARDS/HONORS}

- Academic Distinction Award, Queensland University of Technology International College (QUTIC), 2010

- Distinguished Future Engineers Scholarship, University of Kassel, 2013

- Nominated for the best final year project in Civil Engineering Department, Queensland University of Technology, 2014

- Student Presentation Competition Award, (IHEEP) International Highway Engineering Exchange Program, 2016

\section{AREAS OF INTEREST/SPECIALIZATION}

- Intelligent Transport Systems (ITS)

○ Connected

Vehicles

- Autonomous

Vehicles

- Traffic Operation

○ Traffic Flow Theory

- Intersections

$\circ$ Interchanges

○ Traffic Control

- Traffic Microsimulation

- Geographic Information System (GIS)

- Network Planning and Rout

- Optimization GPS in Transportation

- Sustainability in Transportation

- Mode Choice

- Transportation Policies

- Public Transportation

- Planning and Operation

- Airport Ground Access

\section{SKILLS}

- HCS 2010 (Traffic Operation)

- ArcGIS (GIS Analysis)

- R (statistical programming)

- VISSIM (Simulation and Modeling) 
- $\mathrm{C}++$

\section{AFFILIATIONS}

- Member, Engineers Australia, Since 2014

- Member, American Society of Civil Engineers (ASCE), Since 2015

\section{CONFERENCES}

- TRB 95th Annual Meeting (Washington, DC)

- Transportation Research Forum 57th Annual Meeting (Toronto, Canada)

- 58th Annual Highway Exchange Program Conference, IHEEP 2016 (Helena, MT)

- 2016 ASCE Kentucky Section Annual Meeting (Lexington, KY)

- Ohio Transporation Engineering Conference, OTEC 2016 (Columbus, OH) PRESENTATIONS

- Smart Work Zone Merge System Enabled by Connected and Autonomous Vehicles (IHEEP 2016)

- Smart Work Zone Merge System Enabled by Connected and Autonomous Vehicles (ASCE KY 2016) Improved Emission Estimation at Intersections - A Roundabout Study (OTEC 2016)

- Automated Extraction of Horizontal Curve Data From GIS Roadway Maps (OTEC 2016) 